\title{
Nrf2 Deficiency Attenuates Testosterone Efficiency in Ameliorating Mitochondrial Function of the Substantia Nigra in Aged Male Mice
}

\author{
Baoliang Ren $\mathbb{D}^{1},{ }^{1}$ Tianyun Zhang $\mathbb{D}^{1},{ }^{1}$ Qiqing Guo $\mathbb{D}^{1},{ }^{1}$ Jing Che $\mathbb{D}^{2},{ }^{2}$ Yunxiao Kang $\mathbb{D},{ }^{1,3}$ \\ Rui Cui $\mathbb{D},{ }^{3}$ Yu Wang $\mathbb{D},{ }^{1,3}$ Xiaoming Ji $\mathbb{D},{ }^{1,3}$ Guoliang Zhang $\mathbb{D},{ }^{3}$ and Geming Shi $\mathbb{D}^{1,3,4}$ \\ ${ }^{1}$ Laboratory of Neurobiology, Hebei Medical University, Shijiazhuang 050017, China \\ ${ }^{2}$ Department of Neurology, Affiliated Hospital of Hebei University, Baoding 071000, China \\ ${ }^{3}$ Neuroscience Research Center, Hebei Medical University, Shijiazhuang 050017, China \\ ${ }^{4}$ Hebei Key Laboratory of Neurodegenerative Disease Mechanism, Hebei Medical University, Shijiazhuang 050017, China
}

Correspondence should be addressed to Geming Shi; shigeming@163.com

Received 9 October 2021; Revised 18 January 2022; Accepted 27 January 2022; Published 18 February 2022

Academic Editor: Claudio Cabello-Verrugio

Copyright (c) 2022 Baoliang Ren et al. This is an open access article distributed under the Creative Commons Attribution License, which permits unrestricted use, distribution, and reproduction in any medium, provided the original work is properly cited.

\begin{abstract}
Reduced testosterone level is a common feature of aging in men. Aging, as a risk factor for several neurodegenerative disorders, shows declined mitochondrial function and downregulated mitochondrial biogenesis and mitochondrial dynamics. Mitochondrial biogenesis and mitochondrial dynamics are crucial in maintaining proper mitochondrial function. Supplementation with testosterone is conducive to improving mitochondrial function of males during aging. Nuclear factor erythroid 2-related factor 2 (Nrf2), a regulator of redox homeostasis, is involved in the ameliorative effects of testosterone supplementation upon aging. To explore Nrf2 role in the effects of testosterone supplementation on mitochondrial function during aging, we studied the efficiency of testosterone supplementation in improving mitochondrial function of Nrf2 knockout- (KO-) aged male mice by analyzing the changes of mitochondrial biogenesis and mitochondrial dynamics. It was found that wild-type- (WT-) aged male mice showed low mitochondrial function and expression levels of PGC- $1 \alpha$, NRF-1 $\backslash$ NRF-2, and TFAM regulating mitochondrial biogenesis, as well as Drp1, Mfn1, and OPA1 controlling mitochondrial dynamics in the substantia nigra (SN). $\mathrm{Nrf2} \mathrm{KO}$ aggravated the defects above in $\mathrm{SN}$ of aged male mice. Testosterone supplementation to WT-aged male mice significantly ameliorated mitochondrial function and upregulated mitochondrial biogenesis and mitochondrial dynamics, which were not shown in Nrf2 KO-aged male mice due to Nrf2 deficiency. Testosterone deficiency by gonadectomy (GDX) decreased mitochondrial function, downregulated mitochondrial biogenesis, and altered mitochondrial dynamics balance in young male mice. Supplementation with testosterone to Nrf2 KO-GDX mice only ameliorated the alterations above but did not reverse them to sham level. Nrf2 deficiency attenuated testosterone efficiency in ameliorating mitochondrial function in the $\mathrm{SN}$ of aged male mice through mitochondrial biogenesis and mitochondrial dynamics to some extent. Activation of Nrf2 might contribute to testosterone-upregulating mitochondrial biogenesis and mitochondrial dynamics in the SN during aging to produce efficient mitochondria for ATP production.
\end{abstract}

\section{Introduction}

Aging, as a risk factor for several neurodegenerative disorders including Parkinson's disease (PD) and Alzheimer's disease, shows mild to severe mitochondrial dysfunction. Mitochondrial dysfunction, such as the decreased oxidative phosphorylation, the increased reactive oxygen species
(ROS), and oxidative damage, is found in aged subjects and subjects with aging-related neurodegeneration [1]. As an energy powerhouse, mitochondria are the main producer of ROS in the cells [2,3]. While ROS are involved in normal cellular function, the overproduction of ROS disturbs redox homeostasis and contributes to the brain aging and agingrelated neurodegeneration by oxidating the biomolecules 
of neurons [2]. Due to obvious involvement in aging and aging-related neurodegeneration, the mitochondrion is identified as a major target for neuroprotection [4]. The reduction of oxidative damage to mitochondria by enhancing antioxidative capability and the preservation of the normal mitochondrial function via regulating mitochondrial biogenesis or mitochondrial dynamics have been proposed as strategies to mitigate aging and aging-related neurodegenerative disease $[3,5]$.

Mitochondrial biogenesis is a complex process, during which new mitochondria are formed from preexisting mitochondria through mechanisms involving interaction between the genetic systems of the nucleus and the mitochondria in the cells $[4,6]$. Mitochondrial dynamics is another modification process of mitochondrial morphological state through alterations in mitochondrial fission and fusion activities [7]. Both of them are crucial in the maintenance of mitochondrial function to adapt to energy demands and in the regulation of cell metabolism and antioxidant defense [5, 8]. Nuclear factor erythroid 2-related factor 2 (Nrf2) is an important transcription factor controlling the levels of oxygen free radicals. Activation of the Nrf2antioxidant response elements (ARE) can alleviate pathophysiological processes of neurodegeneration by reducing oxidative stress. Disruption of the Nrf2-ARE pathway results in an increased vulnerability to oxidant neurotoxin [9]. In addition to enhancing redox defense, the Nrf2-ARE pathway facilitates mitochondrial homeostasis and bioenergetics by regulating mitochondrial biogenesis [10] and maintaining balance of mitochondrial dynamics [11-13]. Thus, Nrf2 might be crucial target that affects the efficacy of the intervention strategy imposed on aging and age-related neurodegeneration through manipulating mitochondria.

Previous studies found that testosterone supplementation significantly ameliorates motor behavioral decline, enhances mesodopaminergic activity, and alleviates oxidative damage to the substantia nigra $(\mathrm{SN})$ in aged male rats $[14,15]$. Clinical data also showed that the supplementation with testosterone improves both motor symptom and nonmotor symptom of PD men to some extent [16]. The ameliorative effects of testosterone supplementation above might be related to the alteration of mitochondrial biogenesis and mitochondrial dynamics in the process of testosterone treatment $[17,18]$. Gonadectomy to adult male rats decreases the gene expression of peroxisome proliferatoractivated receptor $\gamma$ coactivator $1 \alpha$ (PGC- $1 \alpha)$, which is a master regulator of mitochondrial biogenesis, in the hippocampus, and induces a significant reduction of mitochondrial DNA-encoded subunits and a marked elevation of oxidative damage, in the hippocampus and the SN [19, 20]. Orchiectomy upregulates mitochondrial fission protein and downregulates mitochondrial fusion protein in the rat myocardial infarction model, while testosterone replacement reverses these effects of orchiectomy on mitochondrial dynamics in cardiac muscles [17]. Thus, testosterone deficiency is an important factor inducing mitochondrial deficits and brain mitochondrial dysfunction in males [19, 20], the common early events of aging-related neurodegeneration [21]. Supplementation with testosterone in the aging process ameliorates aged-related brain mitochondrial dysfunction [18].

In vitro studies revealed that the oxidative stress status seems to play a crucial role in determining a neuroprotective or neurotoxic role of testosterone supplementation [22]. $\mathrm{Nrf2}$, as a regulator of redox homeostasis and mitochondrial homeostasis, might determine the efficacy in the ameliorative effects of testosterone supplementation on aged-related brain mitochondrial dysfunction in males. Thus, to explore roles of $\mathrm{Nrf} 2$ in the effects of testosterone supplementation on the mitochondrial dysfunction in the aging process, the present study analyzed the alterations of mitochondrial function, mitochondrial biogenesis, and mitochondrial dynamics in the $\mathrm{SN}$, as well as behavioral and dopaminergic parameters related to the $\mathrm{SN}$, in testosterone-supplemented Nrf2 knockout-aged male mice. Furthermore, for comparison, young male mice were also included in the study to determine the effects of testosterone deficiency on the SN under the condition of Nrf2 knockout without aging factor interference.

\section{Materials and Methods}

2.1. Animals and Housing. Male Nrf2 wild-type (WT) ICR mice and Nrf2 knockout (KO) mice were kindly provided by academician Chunyan Li (Neurology Department, Second Hospital of Hebei Medical University). They were genotyped by PCR analysis of tail DNA and housed (3-4 per cage) in an air-conditioned room $\left(22 \pm 2^{\circ} \mathrm{C}\right)$ on a $12 \mathrm{~h}$ light/dark cycle with standard chow and water available ad libitum. All the experimental procedures followed the rules in the "Guidelines for the Care and Use of Mammals in Neuroscience and Behavioral Research" and were approved by the Committee of Institutional Animal Care and Use of Hebei Medical University.

2.2. Experiment 1. The aim of Experiment 1 is to investigate whether Nrf2 deficiency affected testosterone efficiency in ameliorating age-related changes related to the $\mathrm{SN}$ of male mice. Young KO (KO-young), aged $\mathrm{KO}$ (KO-aged), and testosterone propionate- (TP-) supplemented-aged $\mathrm{KO}$ (KOaged-TP) mice were used. For KO-aged-TP mice, the mice received subcutaneous $\mathrm{TP}$ injection $(1 \mathrm{mg} / \mathrm{kg}$ per day) at the age of 21 months and were sacrificed at the age of 23 months. KO-aged mice were subjected to the same treatment using sesame oil. WT mice were treated the same as $\mathrm{KO}$ mice and labeled as WT-young, WT-aged, and WTaged-TP groups, respectively.

2.3. Experiment 2. To eliminate aging factors, young male mice were used to explore the effects of testosterone deficiency and testosterone replacement on the parameters analyzed above in Experiment 1. In Experiment 2, $\mathrm{KO}$ mice were divided into the sham-operated (KO-sham), the gonadectomized (KO-GDX), and the GDX with TP treatment (KO-GDX-TP) mice. For the GDX mice, anesthetized mice were castrated by the removal of the testes, epididymis, and epididymal fat under aseptic conditions. The shamoperated mice experienced the same surgical treatment 
except for the bilateral orchiectomies [23]. For KO-GDX-TP mice, the castrated $\mathrm{KO}$ mice were subcutaneously injected with TP ( $1 \mathrm{mg} / \mathrm{kg}$ per day) at the age of 3 months and were sacrificed at the age of 5 months. KO-GDX mice experienced the same treatment as KO-GDX-TP mice with sesame oil instead of TP. Young WT mice were processed the same as KO mice and grouped into WT-sham, WT-GDX, and WT-GDX-TP groups.

2.4. Open-Field Test. The mice were back or tail-marked and handled for 5 days before the behavioral test. Open-field apparatus $(75 \times 75 \times 30 \mathrm{~cm})$ was placed in a quiet room with illumination of 20 lux. A digital video camera was set up above the arena to record mouse open-field activity. Each mouse was individually placed in the center of the openfield apparatus, and its five-minute open-field activity was recorded for further analysis. Based on a previous study [23], open-field activity related to total path length, exploratory behavior (walking, climbing, rearing, and sniffing), and grooming behavior (latency of grooming, number of grooming, and duration of grooming) was noted and scored in shorthand from the recorded videos by three independent observers (RC, XJ, and GZ) who were blind to the experimental plan. The behavioral data documented by them did not show any interobserver differences (ANOVA, NS).

2.5. Footprint Test. Walking gait of mouse was detected via footprint test. The apparatus for footprint test was a tunnel with $10 \mathrm{~cm}$ wide $\times 50 \mathrm{~cm}$ long $\times 10 \mathrm{~cm}$ high. The bottom of the tunnel was covered with white paper. During the experiment, each mouse with forepaws and hindpaws dipped in red and black ink, respectively, was placed at the brightly lit end of a tunnel, which was dark at its other end. The mouse walked down the tunnel, having a set of colored footprints on the white paper. After that, the paper with footprints was then removed for later analysis of walking gait defects. Three independent observers (RC, XJ, and GZ) blind to the experimental purpose measured the stride length of forelimb as well as hindlimb, and the overlap of footprints between forelimb and hindlimb at the right or left side. Stride length is the mean of the forelimb or hindlimb strides, and overlap is the mean of the distance between forelimb and hindlimb at the right or left side.

2.6. Sample Preparation. For LC-MS/MS, biochemical assay, mitochondrial membrane potential (MMP) detection, quantitative real-time PCR (qPCR), and western blot analysis, mice were sacrificed by decapitation. Their brains were removed quickly, and tissue blocks containing the $\mathrm{SN}$ or caudate putamen $(\mathrm{CPu})$ were dissected on an ice-cold plate under stereomicroscopic observation. And then, they were immediately processed for reduced glutathione/oxidized glutathione (GSH/GSSG), malondialdehyde (MDA), $\mathrm{H}_{2} \mathrm{O}_{2}$, ATP, mitochondrial complexes, citrate synthase (CS), and $\mathrm{MMP}$ assays or stored at $-80^{\circ} \mathrm{C}$ after freezing in liquid nitrogen for LC-MS/MS, qPCR, or western blotting on the experimental purposes. For immunohistochemistry (IH), mice were anesthetized and perfused transcardially by $4 \%$ paraformaldehyde in $0.1 \mathrm{M}$ phosphate buffer ( $\mathrm{PB}, \mathrm{pH} 7.4)$. The tissue blocks containing the $\mathrm{SN}$ were postfixed in the same fixative for $4 \mathrm{~h}$ at $4^{\circ} \mathrm{C}$, dehydrated in graded ethanol, cleared in xylene, and then embedded in paraffin wax. For mitochondrial ultrastructure analysis, mice were anesthetized and perfused transcardially by a fixative containing $2 \%$ paraformaldehyde and $1.25 \%$ glutaraldehyde in $0.1 \mathrm{MPB}$. The mouse brain was removed from the cranial cavity and further dissected under a stereomicroscope to collect the SN tissue block. After three washes in $\mathrm{PB}$, the $\mathrm{SN}$ blocks were postfixed with $1 \%$ osmium tetroxide for $2 \mathrm{~h}$, dehydrated in acetone, and then embedded in Araldite.

2.7. LC-MS/MS Assay. Tissue block containing $\mathrm{CPu}$ was weighed and homogenized in $80 \%$ acetonitrile containing $0.1 \%$ formic acid $(5 \mu \mathrm{L})$ and then processed following a previous study [24]. The homogenates were centrifuged at $14,000 \mathrm{~g}$ for $10 \mathrm{~min}$ at $4^{\circ} \mathrm{C}$. The supernatants were collected and used to determine dopamine (DA), 3,4-dihydroxyphenylacetic acid (DOPAC), and homovanillic acid (HVA) levels as previously described [24]. LC separation was performed on an Agilent 1200 LC system (Agilent, Santa Clara, USA) using a Synergi Fusion-RP C18 column $(50 \mathrm{~mm} \times 3.0 \mathrm{~mm}, 4 \mu \mathrm{m})$ provided by Phenomenex. MS/MS detection was carried out using a 3200 QTRAP $^{\text {TM }}$ LC-MS/MS System (Applied Biosystems, Foster City, CA, USA). The multiple-reaction monitoring mode was used for quantification. The principal validation parameters of the LC-MS/MS are described in a previous study [24].

\subsection{Biochemical Analysis}

2.8.1. GSH/GSSG. The SN tissue blocks were homogenized in a solution provided by the GSH/GSSG kit (Code No. A061-1, Nanjing Jiancheng Bioengineering Institute, China) and then centrifuged at $14,000 \times \mathrm{g}$ for $15 \mathrm{~min}$ at $4^{\circ} \mathrm{C}$. The supernatants from centrifuged homogenates were used to assess the GSH/GSSG ratio following the instructions of the GSH/GSSG kit. GSH and GSSG in the samples were first made to react with 5,5'-dithio-bis-(2-nitrobenzoic acid) (DTNB) to produce a colored reagent. Then, the 2vinylpyridine reagent was added to the sample supernatant to ensure that GSSG is the only form of glutathione that reacts with the DTNB reagent. Lastly, the GSSG level was subtracted from the total GSH levels to yield the concentration of reduced GSH. GSH and GSSG levels in the SN were assessed at $412 \mathrm{~nm}$ by a spectrophotometer.

2.8.2. MDA Levels. MDA was detected based on its reaction with thiobarbituric acid (TBA). For the measurement of MDA levels in the SN, tissue blocks were weighed and homogenized in 10 times $(w / v)$ ice-cold $0.1 \mathrm{M} \mathrm{PB}$ at $\mathrm{pH}$ 7.4. The homogenates were centrifuged at $3000 \mathrm{rpm}$ for $10 \mathrm{~min}$, and the supernatant was processed according to the instructions of an MDA kit (Code No. A003-1, Nanjing Jiancheng Bioengineering Institute, China). The absorbance was read at $532 \mathrm{~nm}$ by a spectrophotometer.

2.8.3. $\mathrm{H}_{2} \mathrm{O}_{2}$ Levels. For the detection of $\mathrm{H}_{2} \mathrm{O}_{2}$ in the mitochondria of the $\mathrm{SN}$, the mitochondria were isolated using the tissue mitochondria isolation kit (Code C3606, Beyotime 
Institute of Biotechnology, China). In brief, SN tissue was homogenized in ice-cold buffer (10 mM HEPES, pH7.5, including $200 \mathrm{mM}$ mannitol, $70 \mathrm{mM}$ sucrose, $1.0 \mathrm{mM}$ EGTA, and $2.0 \mathrm{mg} / \mathrm{mL}$ serum albumin) and centrifuged at $1000 \mathrm{~g}$ at $4^{\circ} \mathrm{C}$ for $10 \mathrm{~min}$. The supernatant was centrifuged again at $3500 \mathrm{~g}$ at $4^{\circ} \mathrm{C}$ for $10 \mathrm{~min}$ to collect a mitochondrial pellet. The levels of $\mathrm{H}_{2} \mathrm{O}_{2}$ in the mitochondria were measured spectrophotometrically at $415 \mathrm{~nm}$ according to the protocol of the detection kit (Cat. No. AKAO009M, Beijing Boxbio Science \& Technology, China).

2.8.4. ATP Levels. For detection of ATP levels, the mitochondria were isolated using the Tissue Mitochondria Isolation Kit (Code C3606, Beyotime Institute of Biotechnology, China). ATP levels were measured in isolated mitochondria using an ATP colorimetric assay kit following the manufacturer's instructions (A095-1-1, Nanjing Jiancheng Biotechnology Institute, China). Total mitochondrial protein samples were incubated with the ATP reaction mixture at $37^{\circ} \mathrm{C}$ for $30 \mathrm{~min}$ and detected at $636 \mathrm{~nm}$ using a microplate reader (BioTek Instruments Inc., Highland Park, USA) [25].

2.8.5. Mitochondrial Complex Activities. Mitochondria from the $\mathrm{SN}$ tissue block were isolated according to the protocol of the detection kits. The activities of mitochondrial complexes I, II, III, IV, and V were measured spectrophotometrically using detection kits for complex I (Cat. No. AKOP005M) at $340 \mathrm{~nm}$, complex II (Cat. No. AKOP006M) at $605 \mathrm{~nm}$, complex III (Cat. No. AKOP007M) at $550 \mathrm{~nm}$, complex IV at $550 \mathrm{~nm}$ (Cat. No. AKOP008M, Beijing Boxbio Science \& Technology), or complex V at $340 \mathrm{~nm}$ (A089-5-1, Nanjing Jiancheng Institute of Biotechnology, China) according to manufacturer's specifications.

2.8.6. Citrate Synthase Assay. CS activity in the SN was estimated based on the reduction of 5, $5^{\prime}$-dithio-bis-(2-nitrobenzoic acid) following the specifications of a citrate synthase kit (Code No. A108, Nanjing Jiancheng Bioengineering Institute, China). The SN tissue blocks were firstly homogenized in 0.01 M ice-cold phosphate buffer saline (PBS, pH 7.4) and centrifuged at $14,000 \times \mathrm{g}$ for $15 \mathrm{~min}$ at $4^{\circ} \mathrm{C}$. And then, the CS activities in the supernatant were measured spectrophotometrically based on the instructions of the citrate synthase kit. Absorption values were obtained spectrophotometrically at $412 \mathrm{~nm}$.

2.9. Mitochondrial Membrane Potential Detection. MMP in the SN was detected by the Rhodamine 123 (Rh123) fluorescence method as described previously [26]. The SN tissue block was homogenized in a balanced salt solution and filtered through a nylon mesh screen. The cells were harvested routinely. Following two washes with ice-cold PBS, the cells were incubated in $\mathrm{Rh} 123$ solution $(10 \mu \mathrm{g} / \mathrm{mL})$ at $37^{\circ} \mathrm{C}$ for $30 \mathrm{~min}$. After being washed and resuspended in $1 \mathrm{~mL}$ PBS, the cells were immediately analyzed by flow cytometry (excitation/emission wavelengths, 488/534 nm). MMP was determined by analyzing the changes in Rh123 fluorescence intensity.
2.10. Quantitative Real-Time PCR Analysis. Total RNA from $\mathrm{SN}$ tissue block was isolated according to the manufacturer's protocol using TRizol reagent (Invitrogen, Carlsbad, CA, USA). $1 \mu \mathrm{g}$ of total RNA was reverse-transcribed using random primers to obtain the first-strand cDNA template. Then, qPCR was performed with $20 \mu \mathrm{L}$ reaction solution containing $0.8 \mu \mathrm{L}$ cDNA (diluted 1:10), $2 \mu \mathrm{L}$-specific primers, and $2 \mathrm{x}$ GoTaq $^{\circledR}$ Green Master Mix (Promega, USA). PCR was performed as follows: an initial cycle at $95^{\circ} \mathrm{C}$ for $10 \mathrm{~min}$, followed by 40 cycles at $95^{\circ} \mathrm{C}$ for $10 \mathrm{~s}, 60^{\circ} \mathrm{C}$ for $20 \mathrm{~s}$, and $72^{\circ} \mathrm{C}$ for $15 \mathrm{~s}$. The melting curves of the PCR products were analyzed to confirm the specificity of amplification. Gene expression of $P G C$ $1 \alpha$, nuclear respiratory factor $1(N R F-1), N R F-2$, mitochondrial transcription factor (TFAM), mitochondrial fission protein dynamin-related protein (Drp1), mitochondrial fusion protein mitofusin $1(M f n 1)$, or optic atrophy protein 1 (OPA1) was analyzed using GAPDH as the internal control. For all samples, qPCR was performed in triplicate. Relative quantification was performed using the $2^{-\Delta \Delta \mathrm{Ct}}$ method. The sets of primers were as follows: PGC-1 $\alpha\left(5^{\prime}\right.$-GAAAGGGCC AAACAGAGAGA- $3^{\prime}$ and $5^{\prime}$-GTAAATCACACGGCGC TCTT- $\left.3^{\prime}\right)$, NRF-1 ( $5^{\prime}$-TGGAGTCCAAGATGCTAATG- $3^{\prime}$ and $5^{\prime}$-AGAGCTCCATGCTACTGTTC- $\left.3^{\prime}\right), N R F-2 \quad\left(5^{\prime}\right.$ TCAGTGACTCGGAAATGGAG- $3^{\prime}$ and $5^{\prime}$-TTCACGCAT AGGAGCACTGT-3'), TFAM (5'-CAGGAGGCAAAGGA TGATTC- $3^{\prime}$ and $5^{\prime}$-CCAAGACTTCATTTCATTGTCG-3' ), Drp1 (5' -CAGGAATTGTTACGGTTCCCTAA- $3^{\prime}$ and $5^{\prime}$ CCTGAATTAACTTGTCCCGTGA- $\left.3^{\prime}\right), \quad$ Mfn1 $\quad\left(5^{\prime}\right.$-AACT TGATCGAATAGCATCCGAG- $3^{\prime}$ and $5^{\prime}$-GCATTGCAT TGATGACAGAGC- $\left.3^{\prime}\right)$, OPA1 (5'-GATGACACGCTCTC CAGTGA- $3^{\prime}$ and $5^{\prime}$-TCGGGGCTAACAGTACAACC- $3^{\prime}$ ), and GAPDH (5' ${ }^{\prime}$-ACTCTTCCACCTTCGATGCC- $3^{\prime}$ and $5^{\prime}$ -TCTTGCTCAGTGTCCTTGCT- $3^{\prime}$ ). Accession numbers of the genes for primers are listed in Table S1.

2.11. Analysis of mtDNA Copy Number. Total DNA was extracted from the SN tissue blocks using an Animal Tissue Genomic DNA kit (ZP307-2, ZOMANBIO, China) according to the manufacturer's protocol. Mitochondrial DNA (mtDNA) copy number was determined by quantifying $16 \mathrm{~S}$ rRNA from mtDNA and nuclear-encoded hexokinase 2 (HK2) gene expression via qPCR. qPCR was carried out with $1 \mu \mathrm{L}$ of sample DNA (diluted $1: 10$ ), $2 \mu \mathrm{L}$ of each specific primer, and 2x All-in-OneTM qPCR Mix (GeneCopoeia Inc., USA) in a final volume of $10 \mu \mathrm{L}$. The primers for $16 \mathrm{~S}$ rRNA and HK2 were used as follows: $16 S$ rRNA ( $5^{\prime}$-CCGC AAGGGAAAGATGAAAGAC- $3^{\prime}$ and $5^{\prime}$-TCGTTTGGTTT CGGGGTTTC- $\left.{ }^{\prime}\right)$ and HK2 (5'-GCCAGCCTCTCCTGAT TTTAGTGT- $3^{\prime}$ and $5^{\prime}$-GGGAACACAAAAGACCTCTTC TGG-3 ${ }^{\prime}$ ). Accession numbers of the genes for primers are listed in Table S1. PCR was performed as follows: an initial cycle at $95^{\circ} \mathrm{C}$ for $15 \mathrm{~min}$, followed by 40 cycles of $95^{\circ} \mathrm{C}$ for $10 \mathrm{~s}, 60^{\circ} \mathrm{C}$ for $20 \mathrm{~s}$, and $72^{\circ} \mathrm{C}$ for $20 \mathrm{~s}$. The melting curves of the PCR products were analyzed to confirm the specificity of amplification. qPCR was performed in triplicate. Relative mtDNA copy number was calculated by the ratio between $16 S r R N A$ and $H K 2$ genes using the $2^{-\Delta \Delta C t}$ method. 
2.12. Western Blot Analysis. The $\mathrm{SN}$ or $\mathrm{CPu}$ tissue block was homogenized in radioimmunoprecipitation assay buffer containing $1 \%$ Triton X-100, 0.1\% SDS, $0.5 \%$ sodium deoxycholate, and protease inhibitors (phenylmethanesulfonyl fluoride $100 \mu \mathrm{g} / \mathrm{mL}$, aprotinin $30 \mu \mathrm{g} / \mathrm{mL}$, and sodium orthovanadate $1 \mathrm{mM}$ ) and sonicated for $4 \times 10 \mathrm{~s}$. After centrifugation at $12,000 \mathrm{~g}$ for $20 \mathrm{~min}$ at $4^{\circ} \mathrm{C}$, the supernatant was collected and stored at $-80^{\circ} \mathrm{C}$ for detection of target protein based on the study needs. The pellets were homogenized in ice-cold lysis buffer (20 mM HEPES, pH 7.9, $400 \mathrm{mM} \mathrm{NaCl}$, $1 \mathrm{mM}$ EDTA, and $0.1 \mathrm{mM}$ EGTA) for $15 \mathrm{~min}$. The homogenate was centrifuged at $12,000 \mathrm{~g}$ for $10 \mathrm{~min}$ at $4^{\circ} \mathrm{C}$, and the supernatant was collected for detection of nuclear Nrf2 protein. Samples from the supernatant were diluted in $5 \mathrm{x}$ sample buffer (50 mM Tris, pH 6.8, 2\% SDS, 10\% glycerol, $0.1 \%$ bromophenol blue, and 5\% $\beta$-mercaptoethanol) and heated for $5 \mathrm{~min}$ at $95^{\circ} \mathrm{C}$ before SDS-PAGE on a $10 \%$ gel and transferred to a PVDF membrane (Millipore). The membrane was incubated for $2 \mathrm{~h}$ with $5 \%$ nonfat dry milk in Tris-buffered saline (TBS) containing $0.05 \%$ Tween-20 (TBST). After being rinsed thrice with TBST, the membrane was incubated overnight with rabbit anti-tyrosine hydroxylase $(\mathrm{TH}) \quad(1: 10,000$, Abcam $)$, rabbit anti-dopamine transporter (DAT) $(1: 1000$, Sigma), rabbit anti-PGC- $1 \alpha$ (1:1000, Abcam), rabbit anti-NRF-1 (1:1000, ABclonal), rabbit anti-NRF-2 (1:1000, ABclonal), rabbit anti-TFAM (1:1000, GeneTex), rabbit anti-Drp1 (1:1000, Cell Signaling Technology), rabbit anti-pDrp1-S616 (1:1000, Affinity), rabbit anti-Mfn1 (1:1000, arigo), rabbit anti-OPA1 (1:1000, GeneTex), mouse anti-Nrf2 (1:500, Santa Cruz Biotechnology), rabbit anti-heme oxygenase 1 (HO-1, $1: 300$, Affinity), rabbit anti- $\beta$-actin $(1: 10,000$, ABclonal), or rabbit anti-H3 $\left(1: 1000\right.$, Arigo) antibody at $4^{\circ} \mathrm{C}$ according to the study purposes. After three washes, the membrane was incubated for $1 \mathrm{~h}$ in IRDye ${ }^{\circledR} 800$-conjugated goat anti-rabbit ( $1: 10,000$; Rockland) or anti-mouse ( $1: 5,000$; Rockland) second antibody. The bands were scanned by an Odyssey infrared scanner (LI-COR Biosciences). The densitometry values of the individual protein were normalized with respect to those of $\beta$-actin or $\mathrm{H} 3$, which was used as the endogenous control. For detecting the oxidation of mitochondrial proteins, mouse anti-3-nitrotyrosine (3-NT) antibody (1:1000, Santa Cruz Biotechnology) or rabbit anti-VDAC $(1: 1000$, ABclonal) was used to incubate the PVDF containing the electroblotted proteins from isolated mitochondrial fractions. After performing the same western blot procedures as described before, 3-NT densitometry values were normalized with those of VDAC that was used as mitochondrial endogenous control.

2.13. Immunohistochemistry. $5 \mu \mathrm{m}$ coronal sections sliced from paraffin-embedded SN tissue blocks were mounted on the slides. After deparaffinization and hydration, the sections were processed for antigen retrieval, inactivation of endogenous peroxidase activity, and incubation in normal serum. Subsequently, the sections were incubated with mouse anti-3-nitrotyrosine (3-NT) antibody (1:100, Santa Cruz Biotechnology) overnight at $4^{\circ} \mathrm{C}$. After washing, the sections were incubated with biotinylated goat anti-mouse
$\operatorname{IgG}(1: 500)$ for $2 \mathrm{~h}$ at room temperature. Following incubation at room temperature in horseradish peroxidaseconjugated streptavidin $(1: 500)$ for $1 \mathrm{~h}$, the sections were stained for $5 \mathrm{~min}$ in a solution containing $0.05 \%$ diaminobenzidine and $0.03 \% \mathrm{H}_{2} \mathrm{O}_{2}$ in $0.05 \mathrm{M}$ Tris- $\mathrm{HCl}$ buffer ( $\mathrm{pH}$ 7.6). A computer-assisted image analysis system (Image-Pro Plus 6.0) was used to measure the average optical density (AOD) and the number of 3-NT immunoreactive (3-NT-ir) positive cells in the SN.

2.14. Mitochondrial Ultrastructure Analysis. Ultrathin sections $(70 \mathrm{~nm})$ were obtained with a microtome (UC-7, Leica, Austria). After staining with uranyl acetate $(10 \mathrm{~min})$ and lead citrate $(5 \mathrm{~min})$, the sections were examined under a transmission electron microscope (Hitachi HT7800, Japan) operated at $80 \mathrm{kV}$. For the electron microscopy (EM) image analyses, the mitochondrial number was counted using Image-Pro Plus 6.0 (Media Cybernetics, USA) at $\times 3000$ magnification, and the mitochondrial ultrastructure was analyzed at $\times 25,000$ magnification.

2.15. Statistics. The data are presented as the mean \pm SD. All the data were analyzed by a two-way ANOVA. If the twoway ANOVA was significant, we performed planned comparisons using one-way analysis of variance (one-way ANOVA) for the comparison of treatment effect among same genotype or using Student's $t$-test for the comparison of genotype effect among same treatment. For one-way ANOVA, Levene's test was applied to test of homogeneity of variance. If homogeneity of variance is equal (the significance of Levene's test is greater than 0.05), go to the homogeneity of variance tests ( $F$-statistic), where $P<0.05$, followed by Tukey's honestly significant difference (Tukey's HSD) post hoc test for multiple comparisons. If homogeneity of variance is unequal (the significance of Levene's test is less than 0.05), go to the test of Welch's $F$ test ( $F^{\prime}$-statistic), where $P<0.05$; the post hoc test between groups were done using the Games-Howell procedure. $P<0.05$ was considered statistically significant.

\section{Results}

3.1. Nrf2 Deficiency Attenuated Testosterone Efficiency in Improving Open-Field Activity and Walking Gait of Aged Male Mice. We first performed open-field test and footprint test to observe the behavioral changes of experimental mice among $\mathrm{KO}$-young, $\mathrm{KO}$-aged, and $\mathrm{KO}$-aged-TP, as well as WT-young, WT-aged, and WT-aged-TP groups. Analysis to them revealed declined total path length, walking, climbing, rearing, and sniffing, as well as decreased stride length and increased overlap of footprints in WTaged mice and $\mathrm{KO}$-aged mice compared with corresponding control of their own $(P<0.01$, Figures $1(\mathrm{a})-1(\mathrm{e})$ and $1(\mathrm{i})-1(\mathrm{l}))$. Further reduction in total path length, walking, climbing, rearing, sniffing, and stride length, as well as further increment in overlap of footprints, was found in $\mathrm{KO}$ aged mice compared with WT-aged mice $(P<0.01)$. The male mice between WT-young and $\mathrm{KO}$-young groups did not show significant difference in above behavioral 


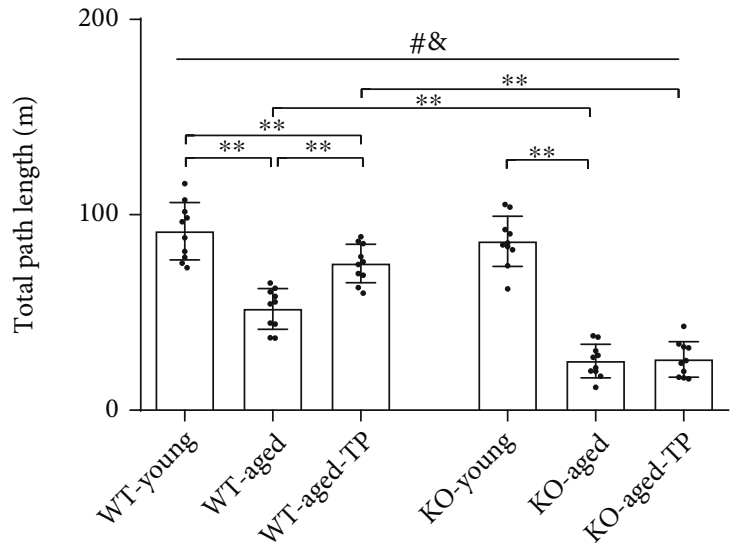

(a)

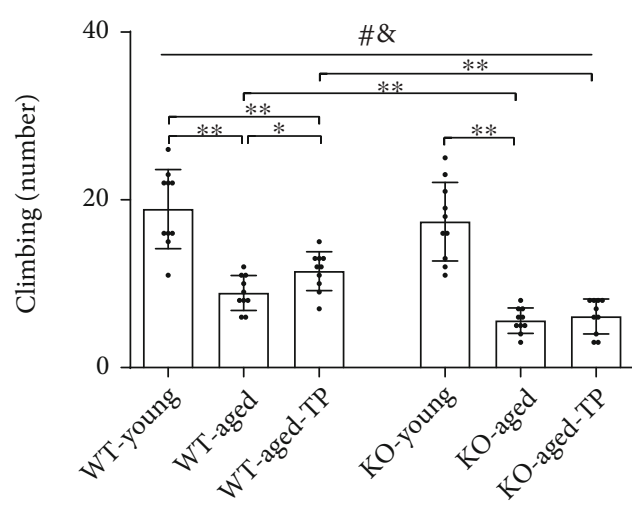

(c)

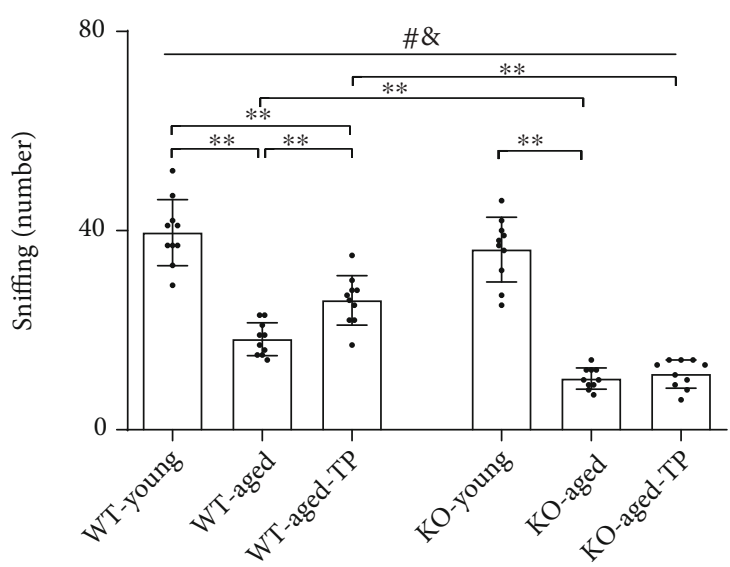

(e)

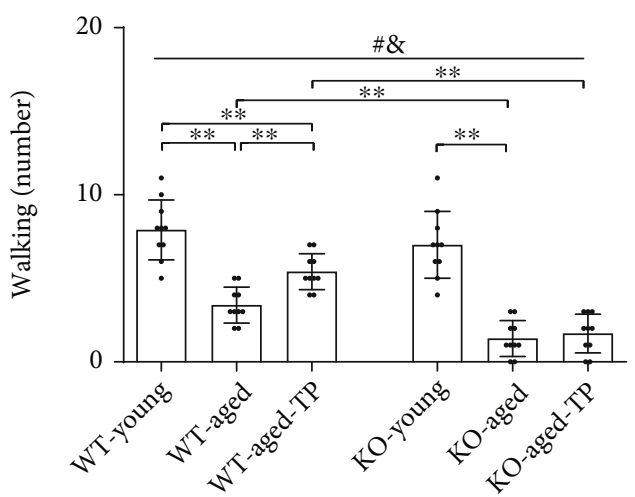

(b)

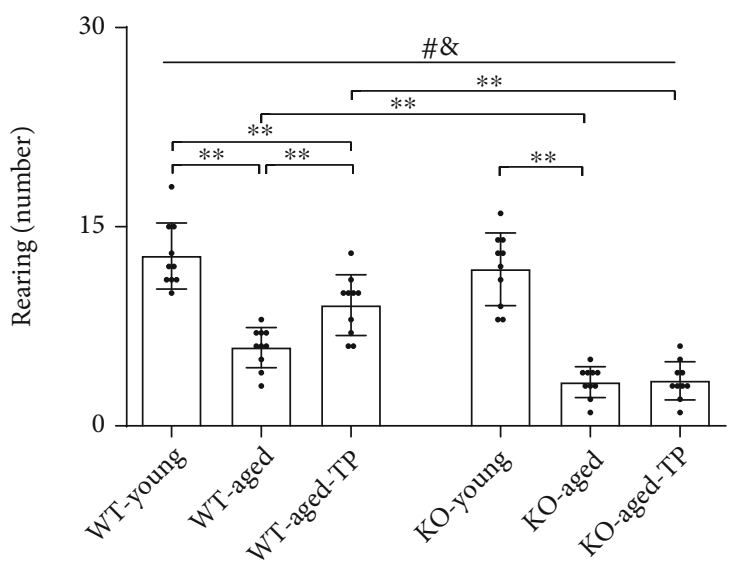

(d)

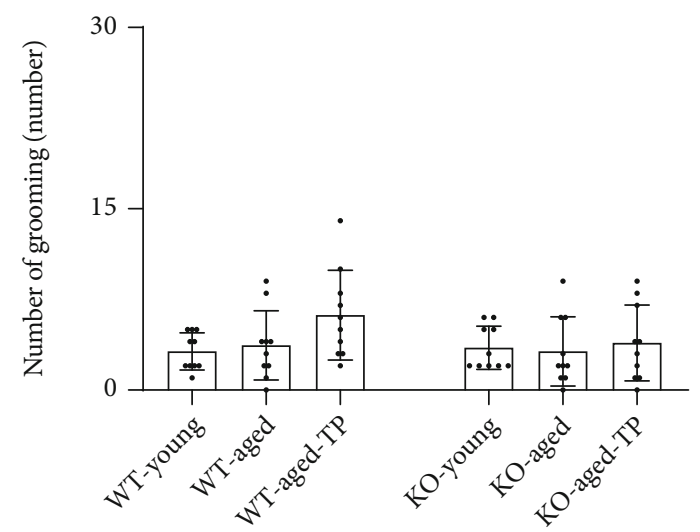

(f)

FIgURE 1: Continued. 


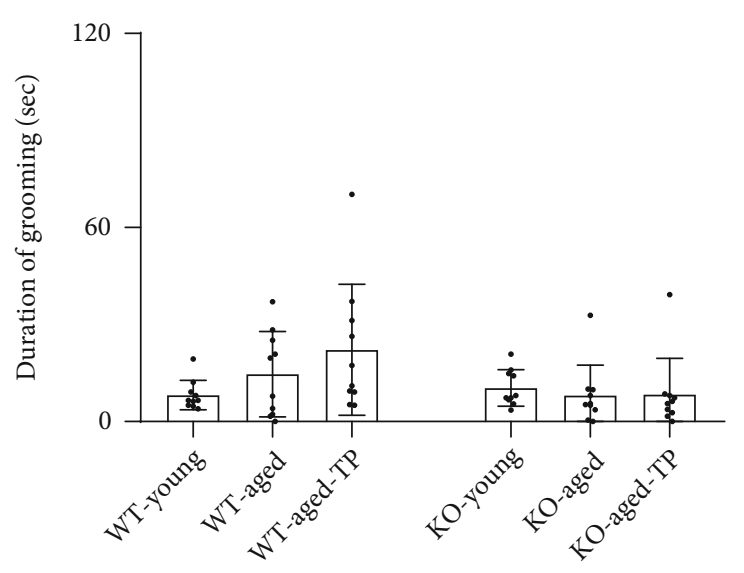

(g)

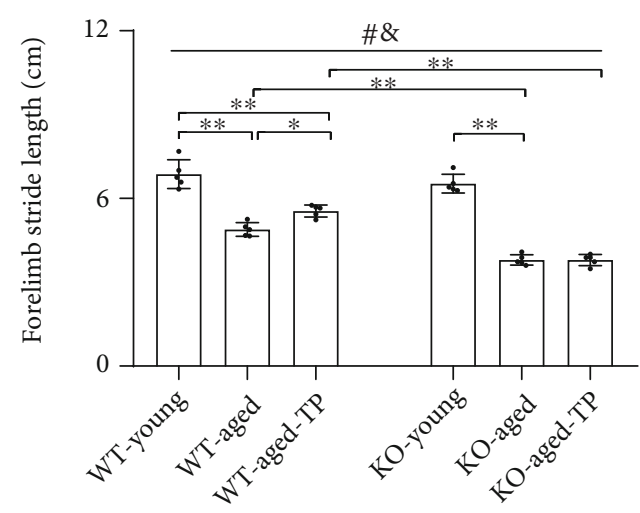

(i)

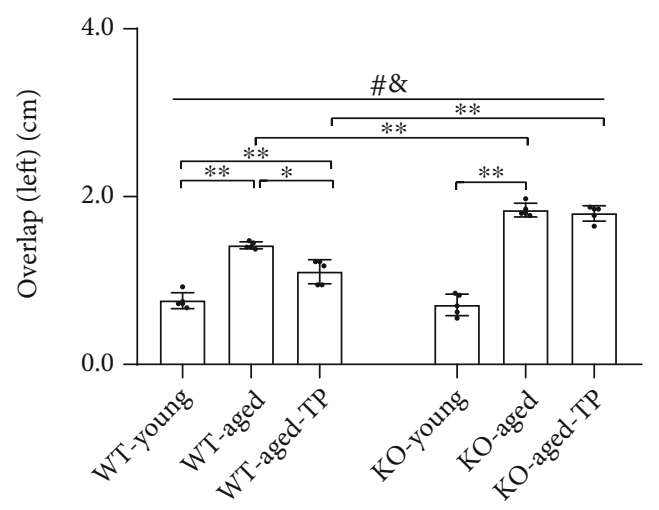

(k)

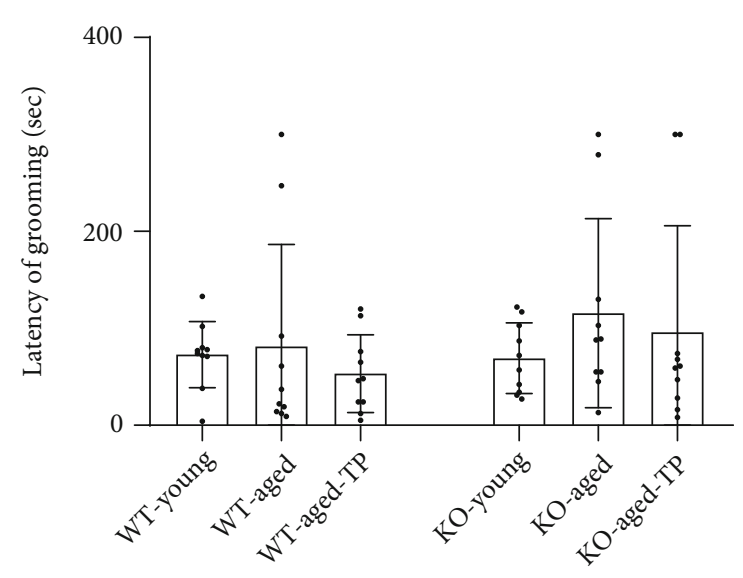

(h)

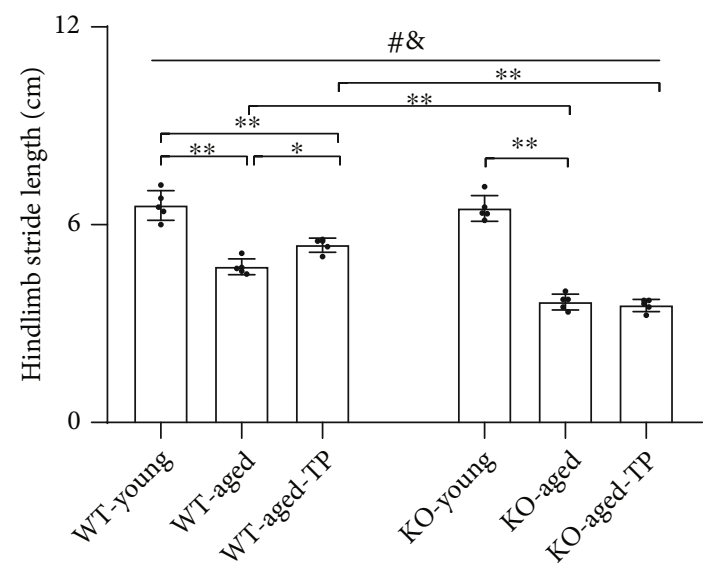

(j)

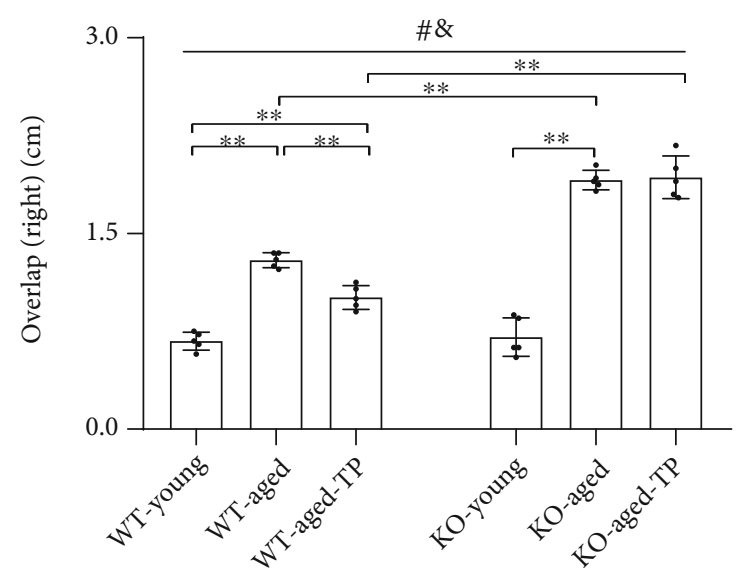

(l)

FIGURE 1: Effects of TP supplementation on open-field activity and walking gait of Nrf2 KO-aged male mice: (a) total path length, (b) walking, (c) climbing, (d) rearing, (e) sniffing, (f) number of grooming, (g) duration of grooming, (h) latency of grooming, (i) forelimb stride length, (j) hindlimb stride length, $(\mathrm{k})$ overlap of left footprints, and (l) overlap of right footprints. Data were presented as mean \pm $\mathrm{SD} ; n=10$ for open-field test; $n=5$ for footprint test. ${ }^{*} P<0.05$ main effect of genotype by two-way ANOVA; ${ }^{\circledR} P<0.05$ main effect of treatment by two-way ANOVA. ${ }^{*} P<0.05$ and ${ }^{* *} P<0.01$.

parameters. TP supplementation to WT-aged mice significantly increased their total path length, walking, climbing, rearing, sniffing, and stride length and decreased overlap of footprints. These effects by $\mathrm{TP}$ were not shown in KO-aged-TP mice. There was no significant intergroup difference among KO-young, KO-aged, KO-aged-TP, WT-young, WT-aged, and WT-aged-TP mice in grooming behavior (Figures 1(f)-1(h)). TP supplementation ameliorated open-field activity and walking gait in WT-aged male mice, but not in $\mathrm{KO}$-aged male mice. 


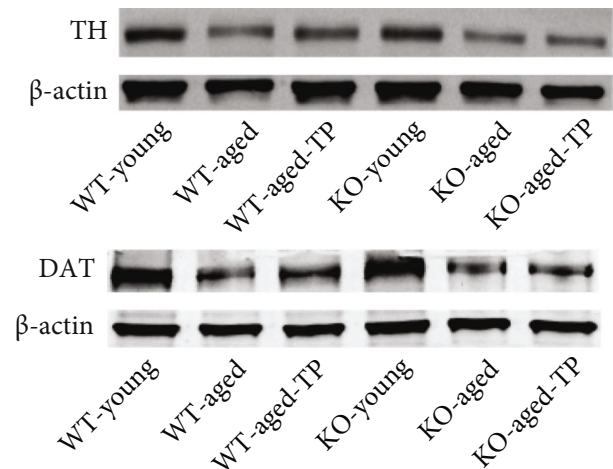

(a)

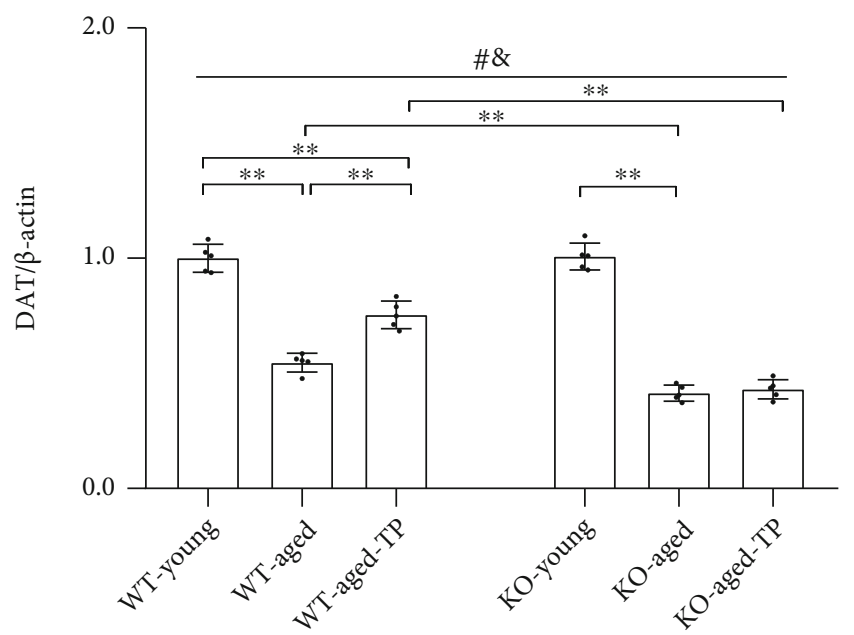

(c)

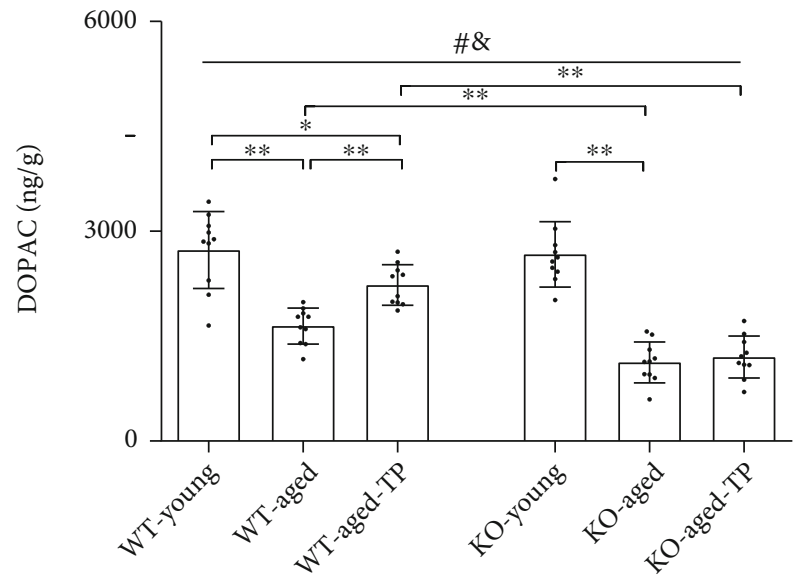

(e)

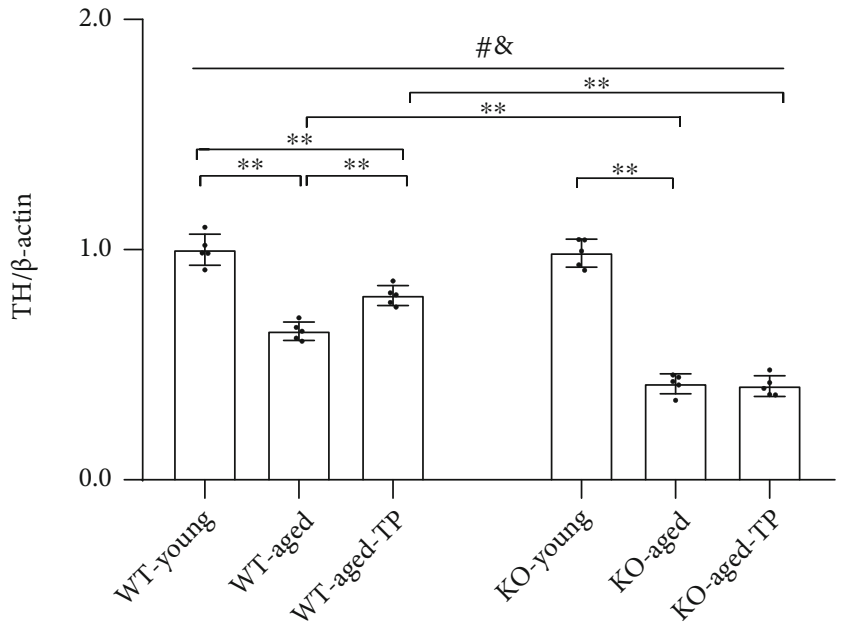

(b)

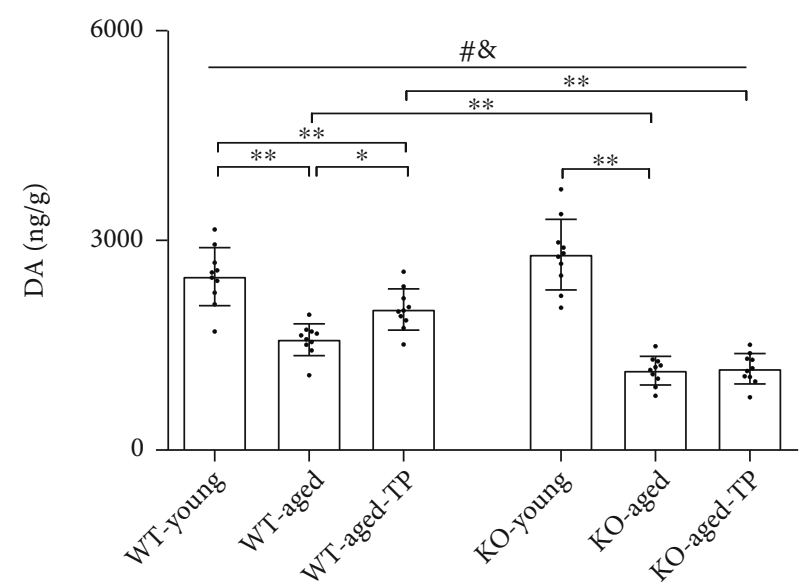

(d)

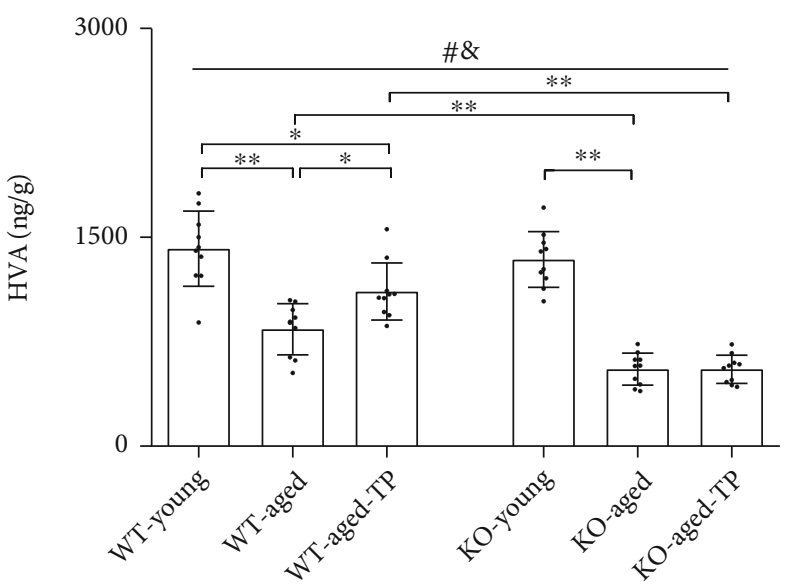

(f)

FIGURE 2: Effects of TP supplementation on dopaminergic activity in the caudate putamen of Nrf2 KO-aged male mice. (a, b) TH and (a, c) DAT were detected by immunoblotting. (d) DA, (e) DOPAC, and (f) HVA were measured by LC-MS/MS assay. Data were presented as mean \pm SD; $n=5$ for TH and DAT; $n=10$ for DA, DOPAC, and HVA. ${ }^{\#} P<0.05$ main effect of genotype by two-way ANOVA; ${ }^{\&} P<0.05$ main effect of treatment by two-way ANOVA. ${ }^{*} P<0.05$ and ${ }^{* *} P<0.01$. 


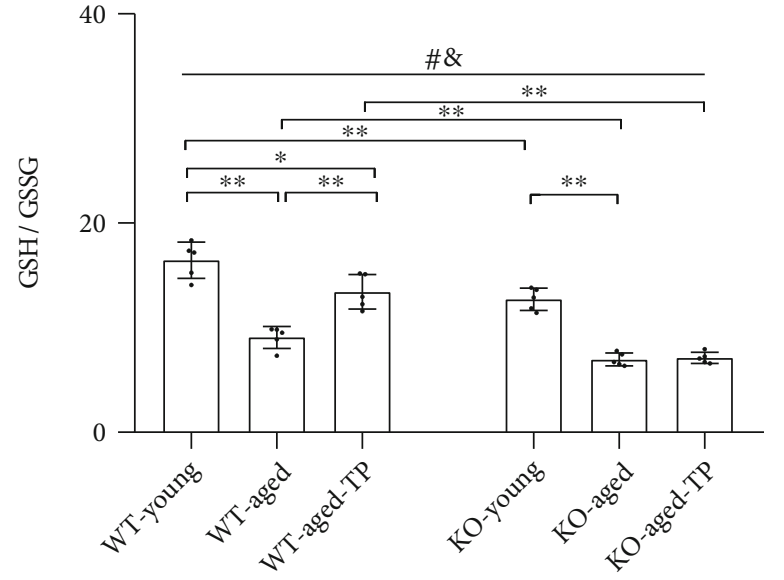

(a)

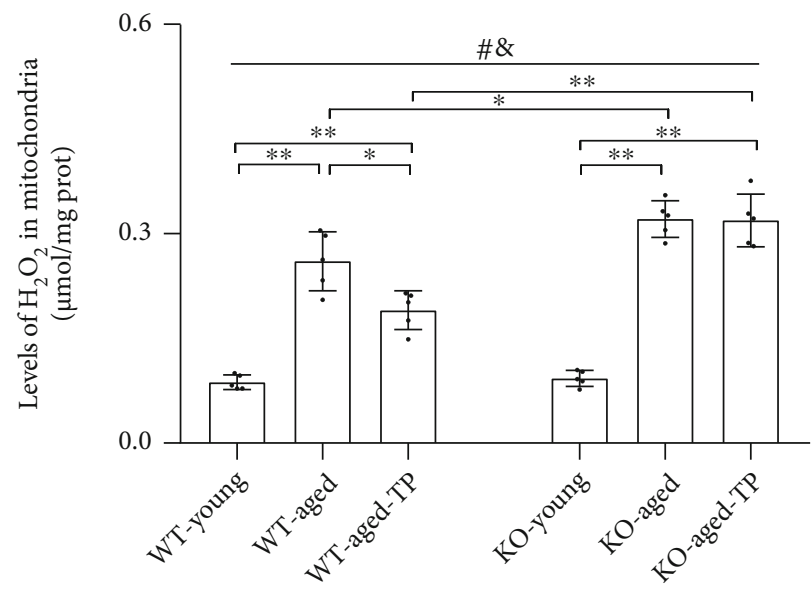

(c)

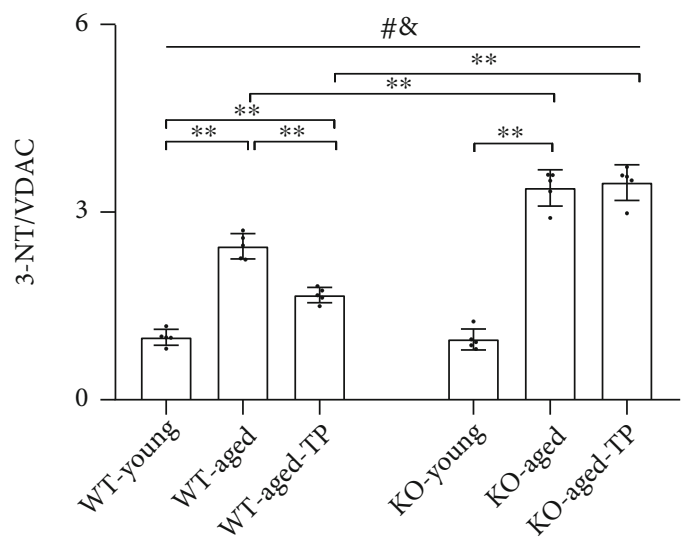

(e)

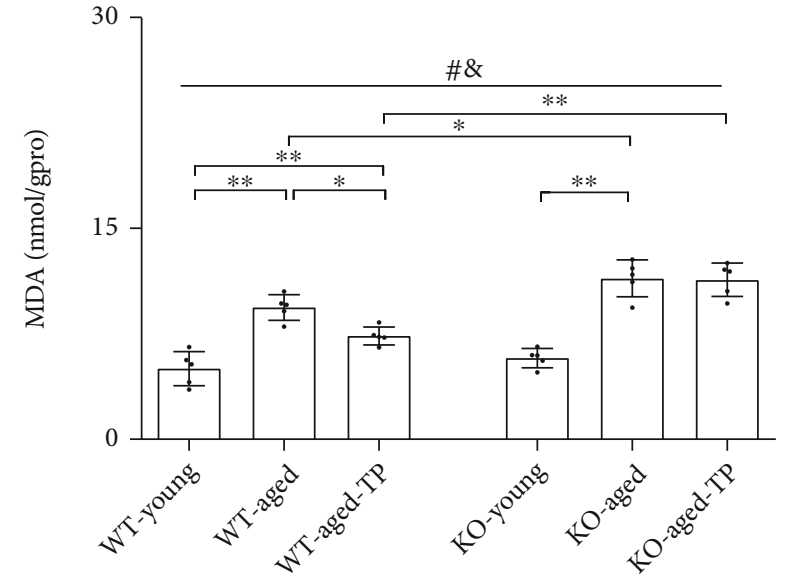

(b)

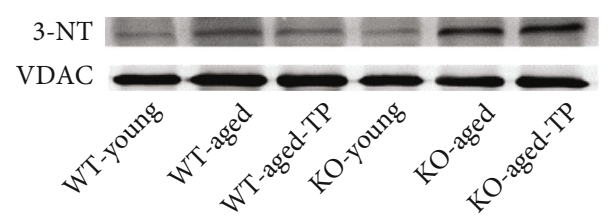

(d)

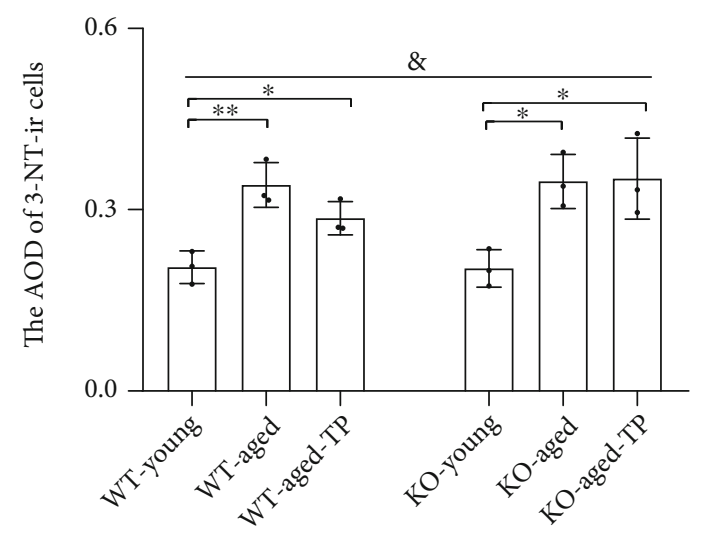

(f)

Figure 3: Continued. 


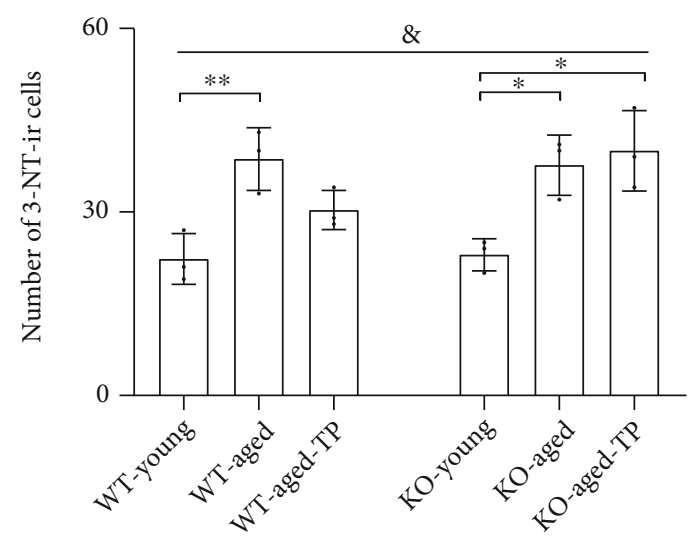

(g)

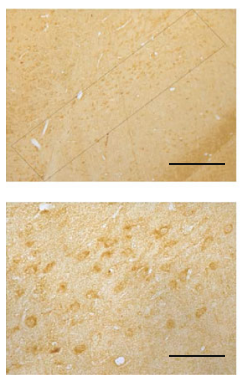

WT-young

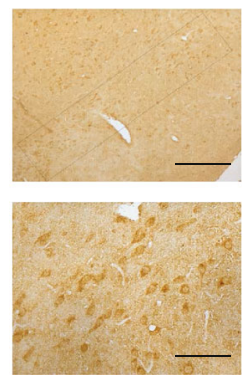

WT-aged

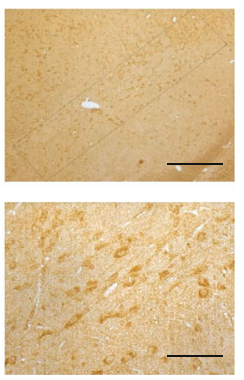

WT-aged-TP

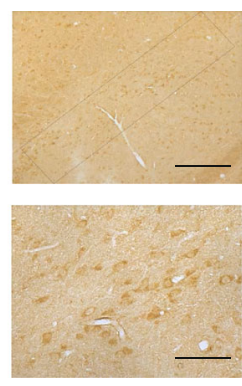

KO-young

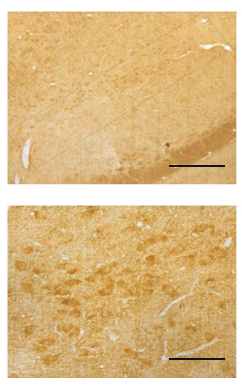

KO-aged

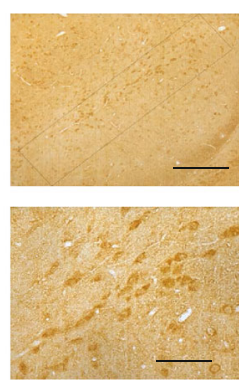

KO-aged-TP

(h)

FIGURE 3: Effects of TP supplementation on oxidative balance in the substantia nigra of Nrf2 KO-aged male mice. (a) GSH/GSSG, (b) MDA, and (c) mitochondrial $\mathrm{H}_{2} \mathrm{O}_{2}$ were assessed by spectrophotometry. (d, e) Mitochondrial 3-NT was measured by immunoblotting; (f-h) 3-NT in the SN was detected by immunohistochemistry. Data were presented as mean \pm SD; $n=5$ for GSH/GSSG, MDA, mitochondrial $\mathrm{H}_{2} \mathrm{O}_{2}$, and mitochondrial 3-NT; $n=3$ for 3-NT immunohistochemistry. Scale bars $=50 \mu \mathrm{m}$ (lower panel); scale bars $=$ $200 \mu \mathrm{m}$ (upper panel). ${ }^{\#} P<0.05$ main effect of genotype by two-way ANOVA; ${ }^{\circledR} P<0.05$ main effect of treatment by two-way ANOVA. ${ }^{*} P<0.05$ and ${ }^{* *} P<0.01$.

3.2. Nrf2 Deficiency Attenuated Testosterone Efficiency in Enhancing Nigrostriatal Dopaminergic Activity of Aged Male Mice. The SN is a brain region rich in dopaminergic neurons. It controls motor behavior and exploratory behavior through a target region in the $\mathrm{CPu}$ [27-29]. Thus, we analyzed the altered status of nigrodopaminergic neurons of aged experimental mice under the Nrf2 deficiency by detecting TH and DAT expression, as well as dopaminergic neurochemical content in the $\mathrm{CPu}$. The levels of $\mathrm{TH}$ and DAT, as well as DA, DOPAC, and HVA in the CPu, were lower in aged male mice of both genotypes than their corresponding young control mice $(P<0.01)$, and they were much lower in $\mathrm{KO}$-aged mice than in WT-aged mice $(P<0.01$, Figures 2(a)-2(f)). There was no difference between WT-young mice and $\mathrm{KO}$-young male mice in the levels of TH and DAT, as well as DA, DOPAC, and HVA in the $\mathrm{CPu}$. Administration of TP significantly increased the expression levels of $\mathrm{TH}$ and DAT, as well as DA, DOPAC, and HVA in the CPu of WT-aged mice. Increased parameters above by $\mathrm{TP}$ were not observed in the $\mathrm{CPu}$ of KO-aged-TP mice. TP supplementation increased TH and DAT expression, as well as dopaminergic neurochemical content in the $\mathrm{CPu}$ of WT-aged male mice, not in $\mathrm{KO}$ aged male mice.
3.3. Nrf2 Deficiency Attenuated Testosterone Efficiency in Ameliorating Oxidative Balance in the SN of Aged Male Mice. Oxidative balance is critically involved in the aging process; therefore, in the SN, we next detected important parameters related to oxidative balance, i.e., GSH/GSSG ratio (a major biomarker of redox status in biological systems), MDA (a marker of ROS-mediated cell membrane damage), and 3-NT (an oxidative stress biomarker of protein nitration) in the tissue, as well as the levels of $\mathrm{H}_{2} \mathrm{O}_{2}$ and 3NT in the mitochondria. Significantly low GSH/GSSG ratio and high MDA and mitochondrial $\mathrm{H}_{2} \mathrm{O}_{2}$ levels in the $\mathrm{SN}$, as well as increased 3-NT levels of the cells and mitochondria in the $\mathrm{SN}$, were found in aged male mice of two genotypes compared with corresponding young male mice (Figures 3(a)-3(h)). Nrf2 KO further lowered GSH/GSSG ratio and elevated $\mathrm{MDA}$ levels as well as mitochondrial $\mathrm{H}_{2} \mathrm{O}_{2}$ and 3-NT levels of aged male mice. Compared with WT-young mice, KO-young mice showed a reduced GSH/ GSSG ratio in the SN $(P<0.01)$. Supplementation with TP significantly increased GSH/GSSG ratio and decreased MDA levels as well as mitochondrial $\mathrm{H}_{2} \mathrm{O}_{2}$ and 3-NT levels of WT-aged male mice. A slight, nonsignificant reduction of 3-NT levels of cells was detected in the SN of WT-aged-TP mice relative to WT-aged mice (Figures $3(\mathrm{f})-3(\mathrm{~h})$ ). 


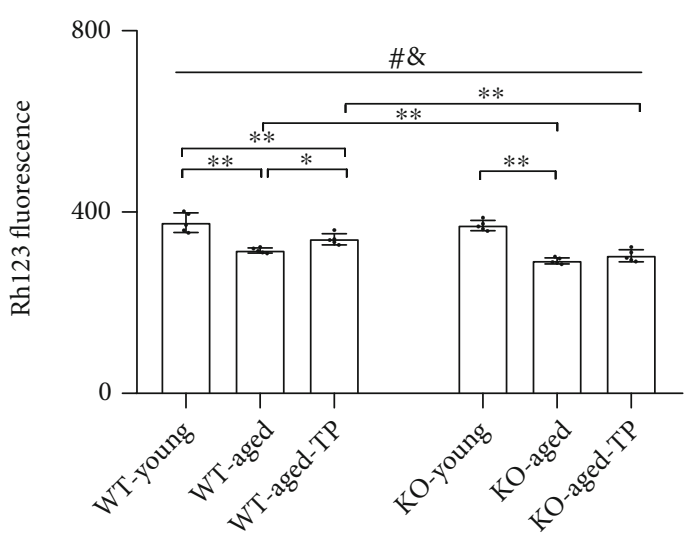

(a)

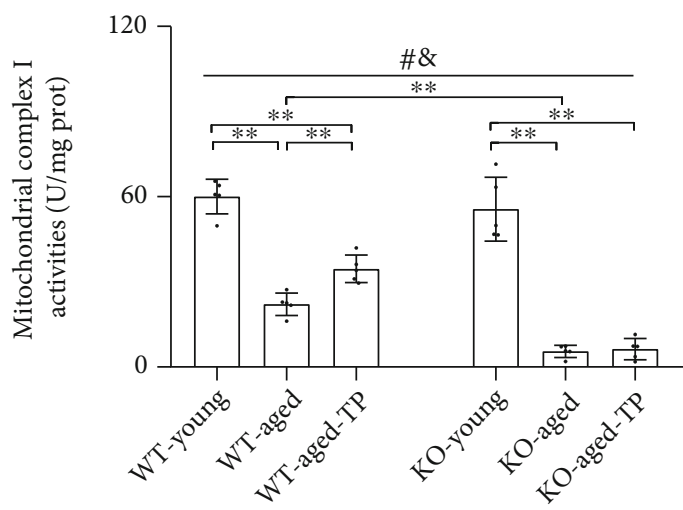

(c)

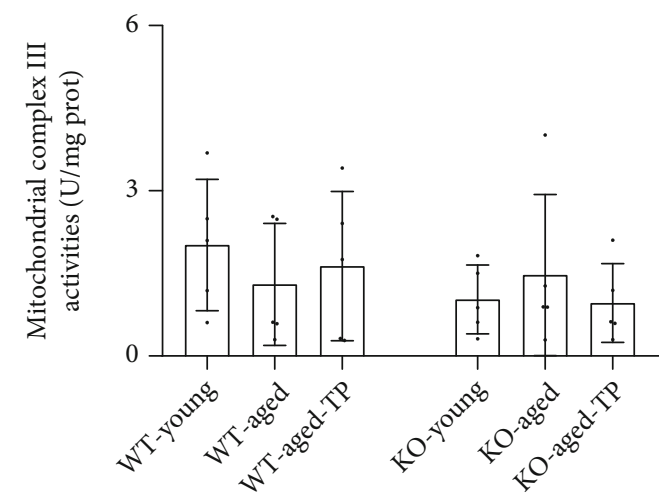

(e)

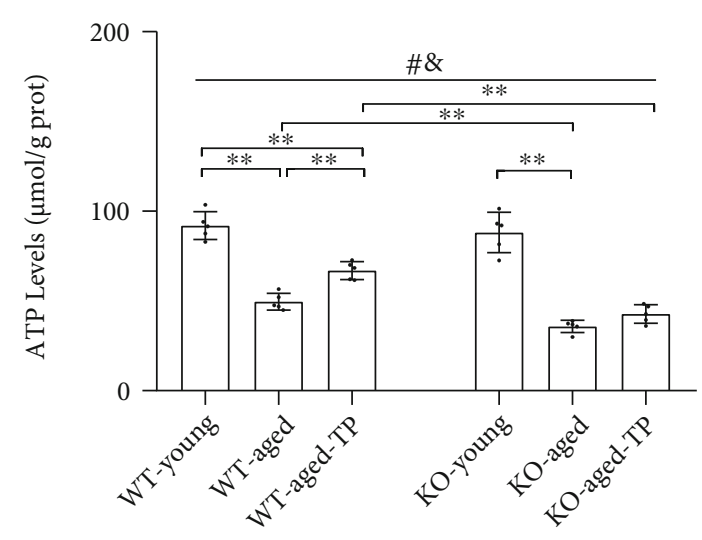

(b)

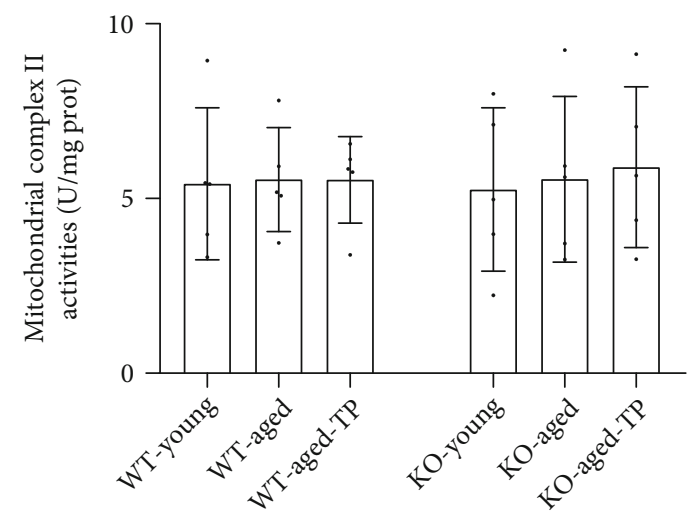

(d)

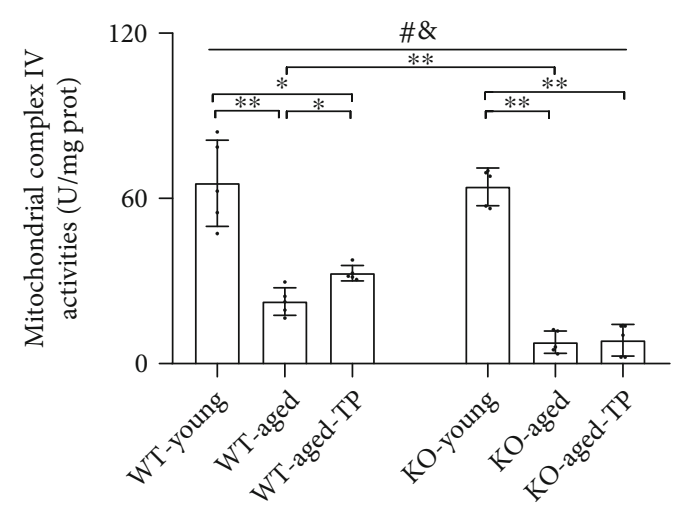

(f)

Figure 4: Continued. 


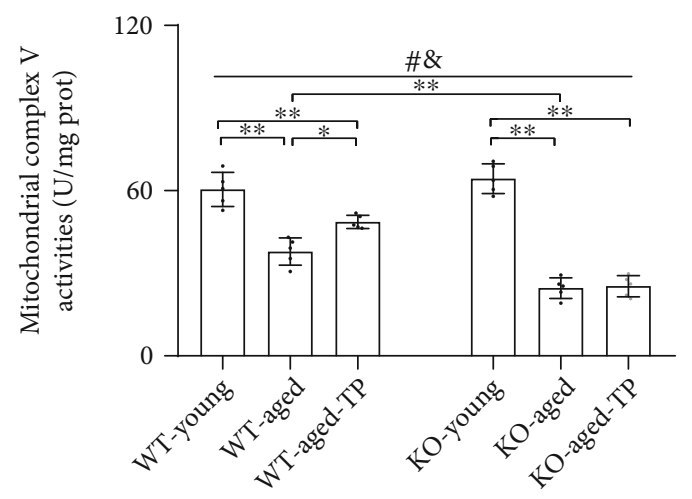

(g)

FIGURE 4: Effects of TP supplementation on mitochondrial function in the substantia nigra of Nrf2 KO-aged male mice. (a) Mitochondrial membrane potential was revealed using the Rh123 fluorescence method. (b) Mitochondrial ATP level, (c) mitochondrial complex I activity, (d) mitochondrial complex II activity, (e) mitochondrial complex III activity, (f) mitochondrial complex IV activity, and (g) mitochondrial complex V activity were revealed by spectrophotometry. Data were presented as mean $\pm \mathrm{SD} ; n=5 .{ }^{\#} P<0.05$ main effect of genotype by twoway ANOVA; ${ }^{\circledR} P<0.05$ main effect of treatment by two-way ANOVA. ${ }^{*} P<0.05$ and ${ }^{* *} P<0.01$.

Increased GSH/GSSG ratio as well as decreased MDA, mitochondrial $\mathrm{H}_{2} \mathrm{O}_{2}$, and 3-NT by TP was not shown in KOaged-TP mice. TP supplementation ameliorated oxidative balance in the SN of WT-aged male mice, not in $\mathrm{KO}$-aged male mice.

3.4. Nrf2 Deficiency Attenuated Testosterone Efficiency in Ameliorating Mitochondrial Function in the SN of Aged Male Mice. As an early event in aging and age-related neurodegenerative diseases, mitochondrial dysfunction leads to insufficient energy and excessive ROS. Healthy neuronal status depends on proper energy supply and oxidative balance. So we further assessed the effects of Nrf2 deficiency on mitochondrial function in the SN of aged male mice during TP supplementation by measuring MMP, ATP levels, and mitochondrial complex activities. Decreased MMP and mitochondrial ATP levels, as well as the activities of mitochondrial complexes I, IV, and V, were detected in the SN of aged male mice of two genotypes compared with corresponding young male mice $(P<0.01$, Figures $4(\mathrm{a})-4(\mathrm{c}), 4(\mathrm{f}$ ), and 4(g)). Nrf2 KO further decreased them in the $\mathrm{SN}$ of aged male mice $(P<0.01)$. No significant difference in MMP and mitochondrial ATP levels, as well as activities of mitochondrial complexes I, IV, and V, was found between WT-young and KO-young mice. Supplementation with TP increased MMP and mitochondrial ATP levels, as well as the activities of mitochondrial complexes I, IV, and $\mathrm{V}$ in the SN of WT-aged male mice. Increased MMP and mitochondrial ATP levels, as well as the activities of mitochondrial complexes I, IV, and V, were not shown in the SN of $\mathrm{KO}$-aged-TP mice. There was no significant intergroup difference among KO-young, KO-aged, KO-aged-TP, WTyoung, WT-aged, and WT-aged-TP mice in the activities of mitochondrial complexes II and III (Figures 4(d) and 4(e)). TP supplementation ameliorated mitochondrial function in the SN of WT-aged male mice, but not in KO-aged male mice.
3.5. Nrf2 Deficiency Attenuated Testosterone Efficiency in Increasing PGC-1 $\alpha$ and Its Downstream Target Expression in the SN of Aged Male Mice. Based on the decreased mitochondrial biogenesis signaling in aging process, the improved mitochondrial function in aging via the induction of mitochondrial biogenesis [30], and the above-found effects of Nrf2 deficiency on mitochondrial function of aged male mice during TP supplementation, we next examined the altered expression of key inducer and effectors of mitochondrial biogenesis, namely, PGC- $1 \alpha$, NRF-1, NRF-2, and TFAM in the SN of experimental mice. Aged male mice of both genotypes showed decreased mRNA levels of $P G C-1 \alpha$, $N R F-1, N R F-2$, and TFAM in the SN relative to respective young control $(P<0.01$, Figures 5(a)-5(d)). Nrf2 KO further reduced their mRNA levels in the $\mathrm{SN}$ of aged male mice $(P<0.01)$. PGC-1 $\alpha, N R F-1, N R F-2$, and TFAM mRNA levels were not significantly different in the $\mathrm{SN}$ of $\mathrm{KO}$-young mice relative to WT-young mice. Supplementation with TP significantly increased $P G C-1 \alpha, N R F-1, N R F-2$, and TFAM mRNA levels in the SN of WT-aged male mice. There was no difference between $\mathrm{KO}$-aged mice and $\mathrm{KO}$-aged-TP mice in the expression levels of $P G C-1 \alpha$ and its downstream targets. Immunoblotting data from PGC-1 $\alpha$, NRF-1, NRF-2, and TFAM agreed with their mRNA changes (Figures 5(e)5(1)). TP supplementation increased the expression levels of PGC- $1 \alpha$ and its downstream targets in the SN of WT-aged male mice, but not in $\mathrm{KO}$-aged male mice.

3.6. Nrf2 Deficiency Attenuated Testosterone Efficiency in Increasing Mitochondrial Content in the SN of Aged Male Mice. Since stimulation of mitochondrial biogenesis is accompanied by increased mitochondrial content, so following, we investigated the effects of Nrf2 deficiency on mitochondrial content in the SN of aged mice supplemented with TP through detecting CS activity (a mitochondrial matrix enzyme), mtDNA copy number, and mitochondrial number. CS activity, mtDNA copy number, and 


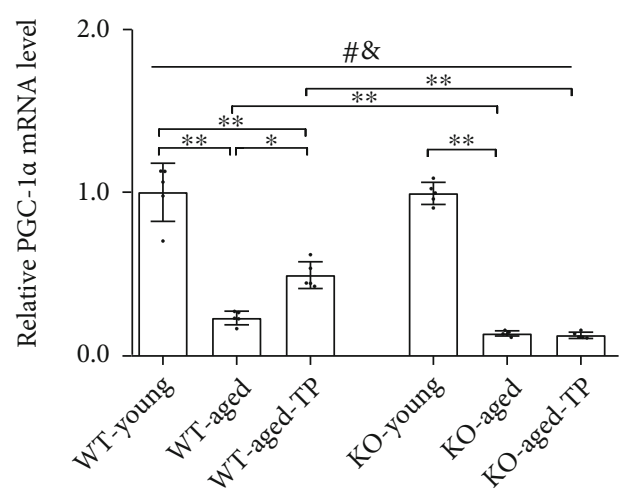

(a)

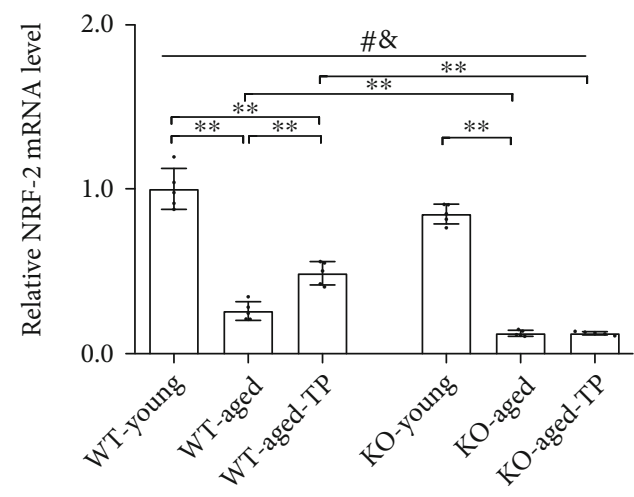

(c)

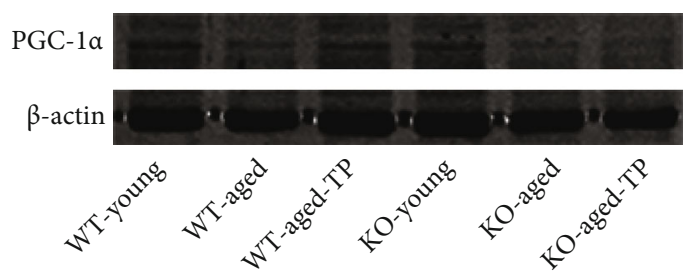

(e)

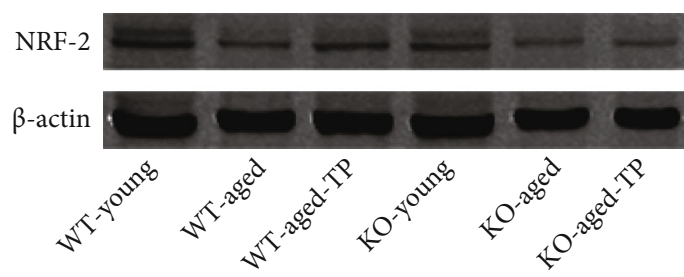

(g)

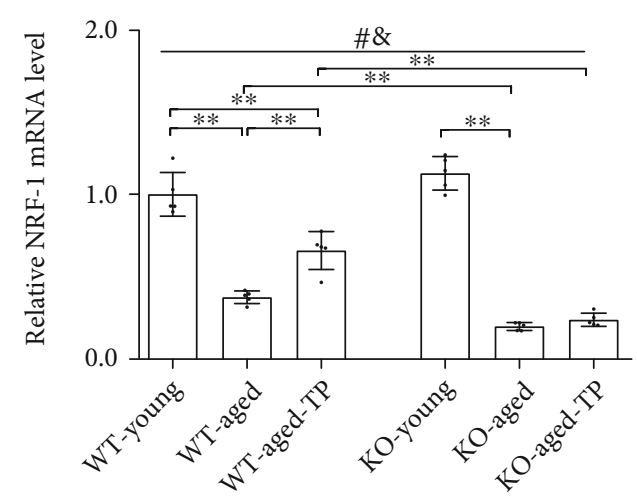

(b)

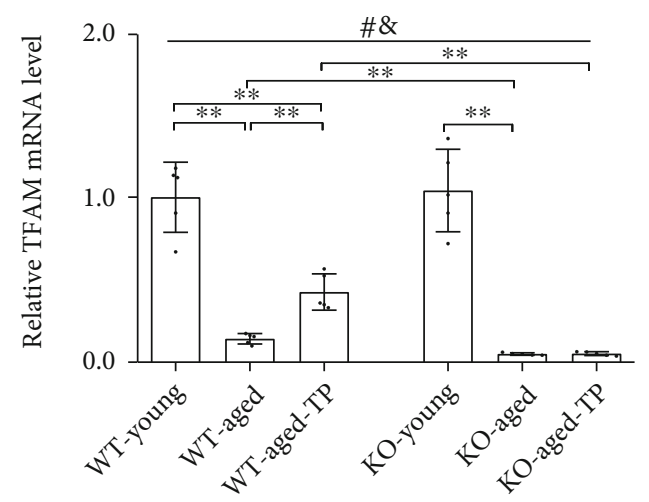

(d)

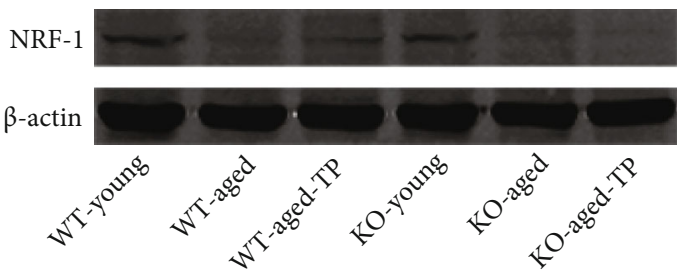

(f)

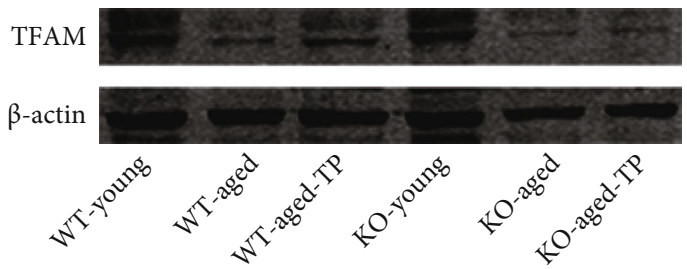

(h)

FIgURe 5: Continued. 


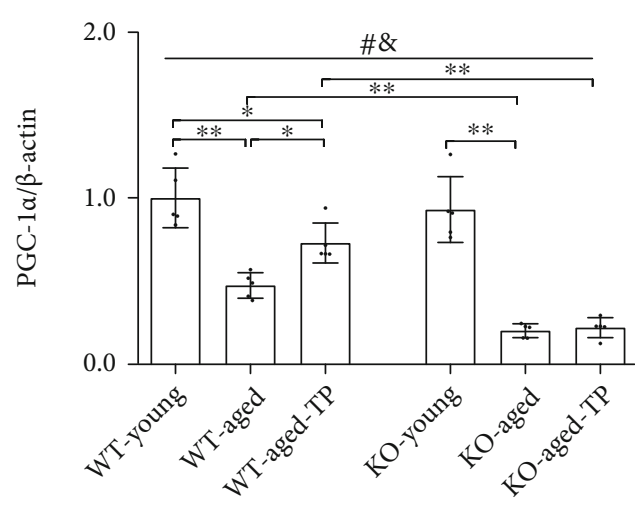

(i)

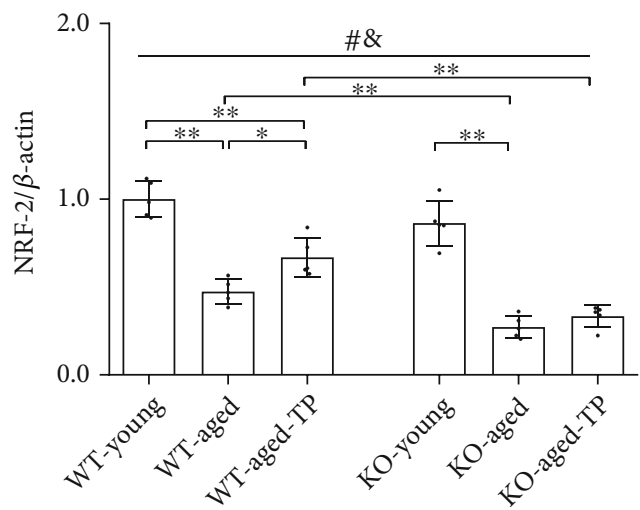

(k)

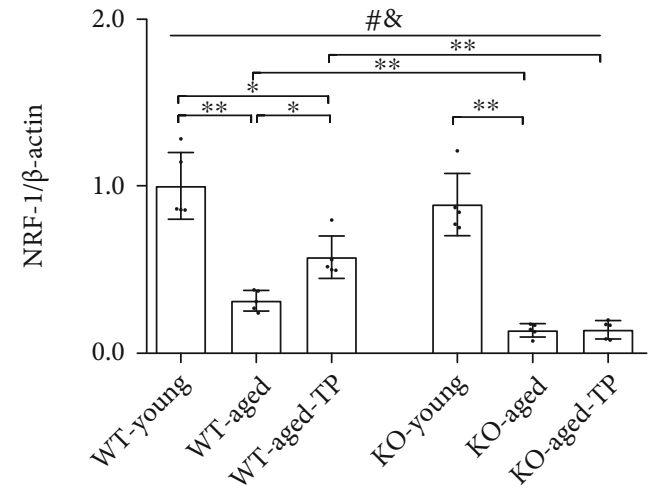

(j)

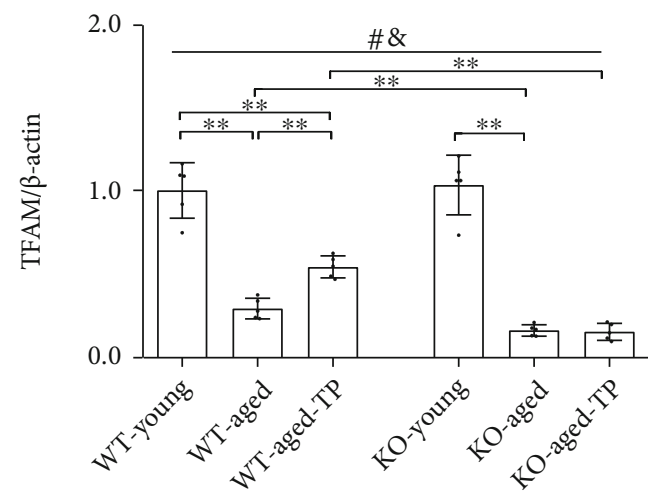

(l)

Figure 5: Effects of TP supplementation on mitochondrial biogenesis in the substantia nigra of Nrf2 KO-aged male mice. (a) $P G C$ - $1 \alpha$, (b) NRF-1, (c) NRF-2, and (d) TFAM mRNAs were revealed by qPCR. (e, i) PGC-1 $\alpha$, (f, j) NRF-1, (g, k) NRF-2, and (h, l) TFAM proteins were detected by immunoblotting. Data were presented as mean $\pm \mathrm{SD} ; n=5$. ${ }^{\sharp} P<0.05$ main effect of genotype by two-way ANOVA; ${ }^{\circledR} P<0.05$ main effect of treatment by two-way ANOVA. ${ }^{*} P<0.05$ and ${ }^{* *} P<0.01$.

mitochondrial number were reduced in the SN of aged male mice of both genotypes relative to their corresponding young control $(P<0.01$, Figures $6(\mathrm{a})-6(\mathrm{c}))$. KO-aged male mice showed much lower CS activity mtDNA copy number and mitochondrial number in the SN than WT-aged male mice. There is no significant difference in CS activity, mtDNA copy number, and mitochondrial number in the SN between KO-young male mice and WT-young male mice. Supplementation with TP increased CS activity, mtDNA copy number, and mitochondrial number in the SN of WT-aged male mice $(P<0.01)$. Increased CS activity, mtDNA copy number, and mitochondrial number by TP were not found in $\mathrm{KO}$-aged-TP mice. In addition, there were striking differences in the mitochondrial ultrastructure among the experimental groups. Compared with mitochondria from the SN of WT-young mice, which presented a normal mitochondrial structure with clear cristae, most mitochondria from the WT-aged or KO-aged male mice showed disorganized cristae. Supplementation with TP to WT-aged male mice improved the ultrastructural alterations of mitochondrial cristae in the $\mathrm{SN}$, which was not observed in KO-aged male mice (Figure 6(d)). TP supplementation increased mitochondrial content in the SN of WT-aged male mice, but not in $\mathrm{KO}$-aged male mice.
3.7. Nrf2 Deficiency Attenuated Testosterone Efficiency in Regulating Levels of Drp1, Mfn1, and OPA1 in the SN of Aged Male Mice. Mitochondrial dynamics take part in maintaining mitochondrial function, and abnormal mitochondrial dynamics are shown in aging and age-related neurodegenerative conditions. Therefore, we analyzed the alterations in the levels of Drp1 and its phosphorylation (pDrp1-S616), Mfn1, and two OPA1 forms (long OPA1: LOPA1 and short OPA1: S-OPA1) in the SN of experimental mice, which are involved in regulating mitochondrial dynamics. Aged male mice of both genotypes showed reduced mRNA levels of Drp1, $M f n 1$, and OPA1 in the SN compared with respective young control $(P<0.01$, Figures $7(\mathrm{a})-7(\mathrm{c}))$. $\mathrm{Nrf} 2 \mathrm{KO}$ further reduced their mRNA levels in the $\mathrm{SN}$ of aged male mice. There was no significant difference between WTyoung and KO-young mice in mRNA levels of Drp1, Mfn1, and OPA1 in the SN. Supplementation with TP increased $\operatorname{Drp} 1, M f n 1$, and OPA1 mRNA levels in the SN of WT-aged male mice. Increased mRNA levels of $\operatorname{Drp} 1, M f n 1$, and OPA 1 by TP were not detected in the $\mathrm{SN}$ of KO-aged-TP mice. Immunoblotting data showed the levels of Drp1, pDrp1-S616, Mfn1, L-OPA1, and S-OPA1 were significantly reduced in the $\mathrm{SN}$ of WT-aged mice relative to WT-young mice (Figures 7(d)-7(i)). They were much lower in KO-aged mice 


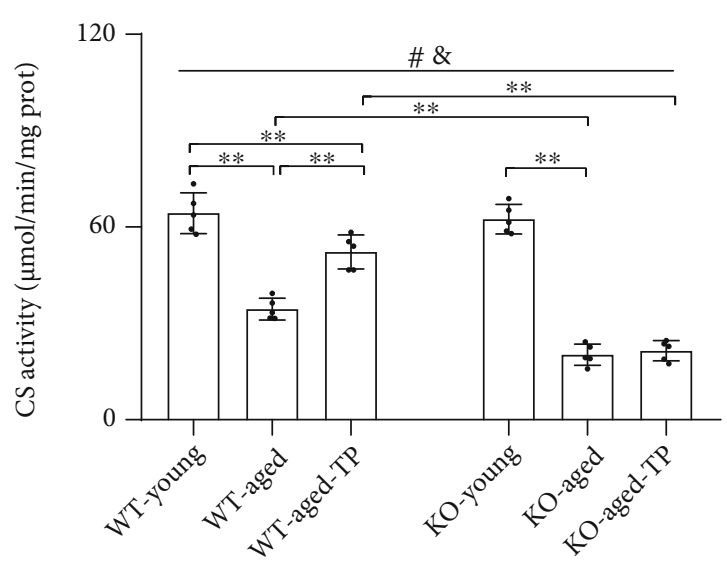

(a)

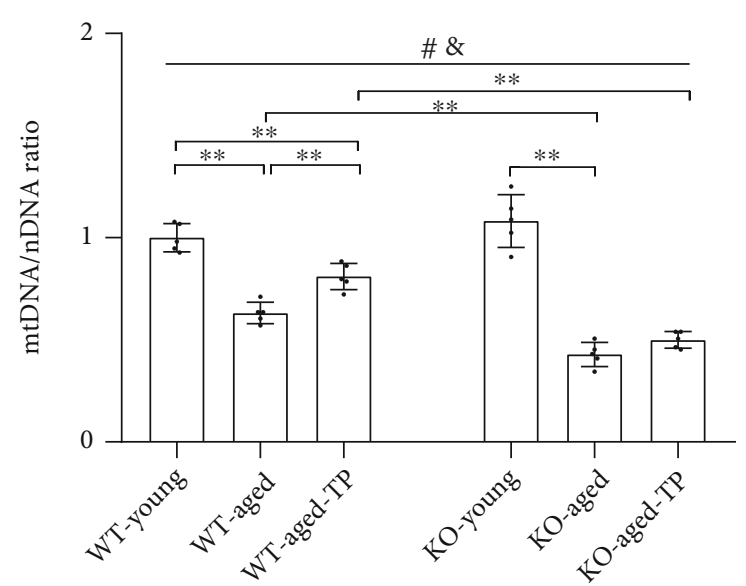

(b)

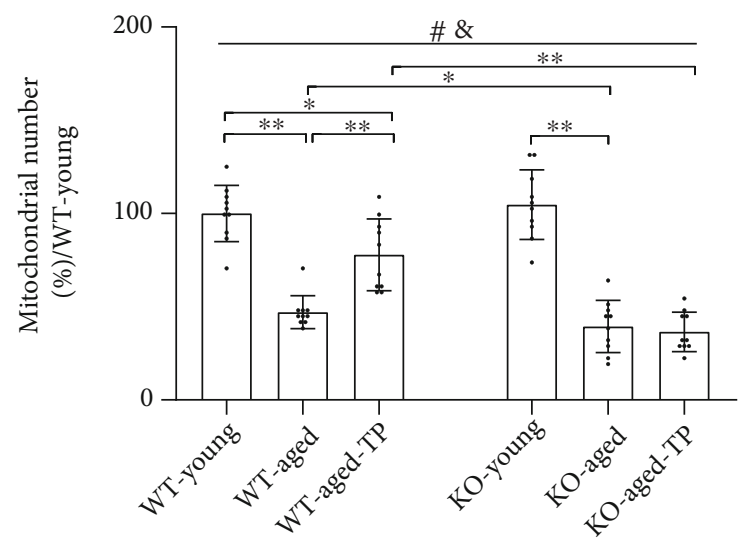

(c)

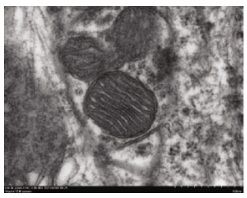

WT-young

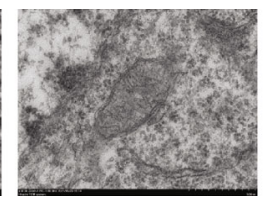

WT-aged

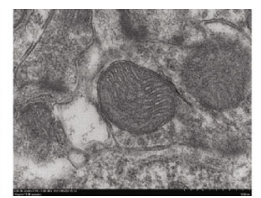

WT-aged-TP

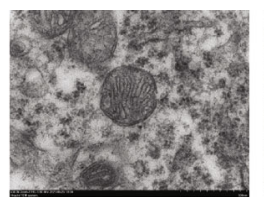

KO-young

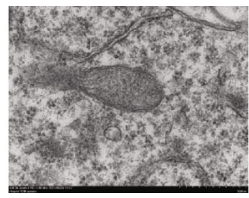

KO-aged

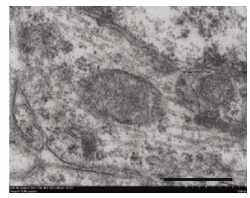

KO-aged-TP

(d)

FIgURE 6: Effects of TP supplementation on mitochondrial content in the substantia nigra of Nrf2 KO-aged male mice. (a) CS activity was assessed by spectrophotometry. (b) mtDNA/nDNA was detected by qPCR. (c) Mitochondrial number was counted using Image-Pro Plus 6.0 by an electron microscope. (d) Mitochondrial ultrastructure images were taken by an electron microscope. Data were presented as mean $\pm \mathrm{SD} ; n=5$ for CS activity and mtDNA/nDNA; $n=2$ for an electron microscope (mitochondrial number from an analysis of 5 images per sample from each group). Scale bar $=500 \mathrm{~nm}$. ${ }^{\#} P<0.05$ main effect of genotype by two-way ANOVA; ${ }^{\circledR} P<0.05$ main effect of treatment by two-way ANOVA. ${ }^{*} P<0.05$ and ${ }^{* *} P<0.01$.

than in WT-aged mice, except for S-OPA1 (Figure 7(i)). Significantly increased levels of Drp1, pDrp1-S616, Mfn1, LOPA1, and S-OPA1 were present in the SN of WT-aged-TP mice compared with WT-aged mice and were not found in $\mathrm{KO}$-aged-TP mice relative to $\mathrm{KO}$-aged mice. TP supplementation increased the levels of Drp1, pDrp1-S616, Mfn1, and two OPA1 forms in the SN of WT-aged male mice, but not in Nrf2 KO-aged male mice.

3.8. The Effects of TP Supplementation on Nrf2 in the SN of Aged Male Mice. To explore whether testosterone induces Nrf2 expression or promotes its nuclear translocation, Nrf2 and its downstream target $\mathrm{HO}-1$ were detected by immuno- blotting. Relative to WT-aged mice, significantly increased Nrf2 levels in the SN as well as in nucleus fraction were detected in WT-aged-TP mice (Figures 8(a), 8(b), 8(d), and $8(\mathrm{e})$ ). Elevated HO-1 levels were shown in the SN of WT-aged-TP mice compared with WT-aged mice $(P<0.01$ ), and increased HO-1 levels in the $\mathrm{SN}$ were not detected in KO-aged-TP mice relative to KO-aged mice. Supplementation with TP increased Nrf2 levels and promoted its nuclear translocation in WT-aged male mice.

3.9. The Effects of Nrf2 Deficiency on the Testosterone Efficiency in GDX Young Male Mice. Under the condition of ruling out aging factor, the above parameters detected 


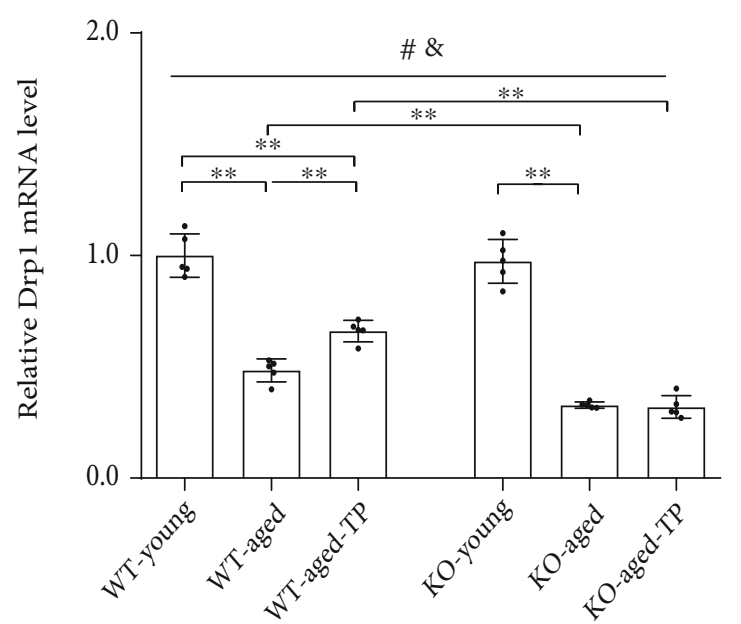

(a)

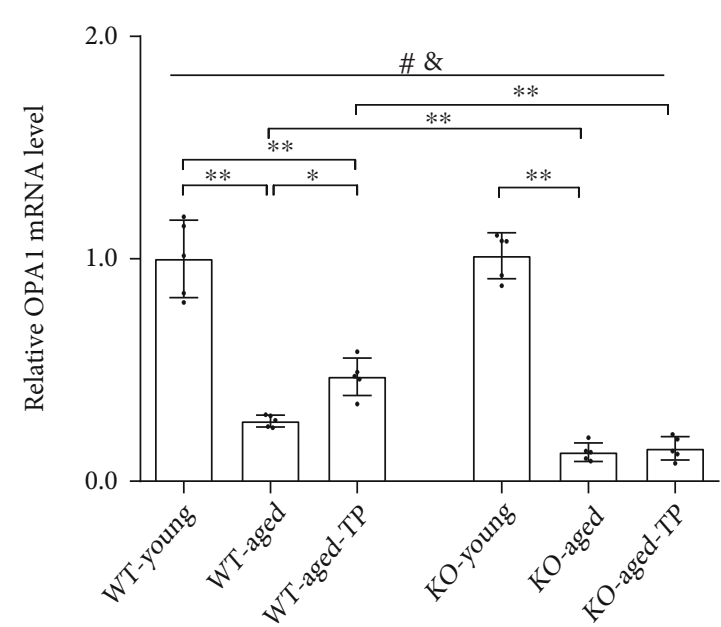

(c)

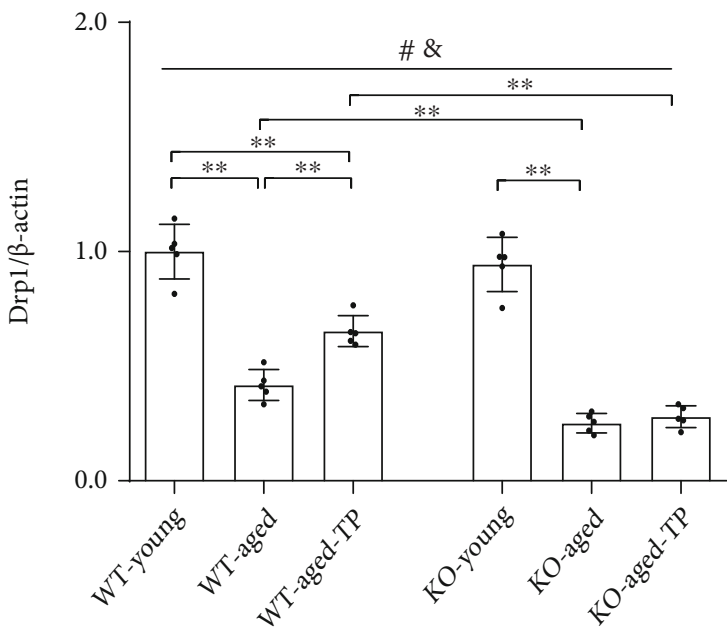

(e)

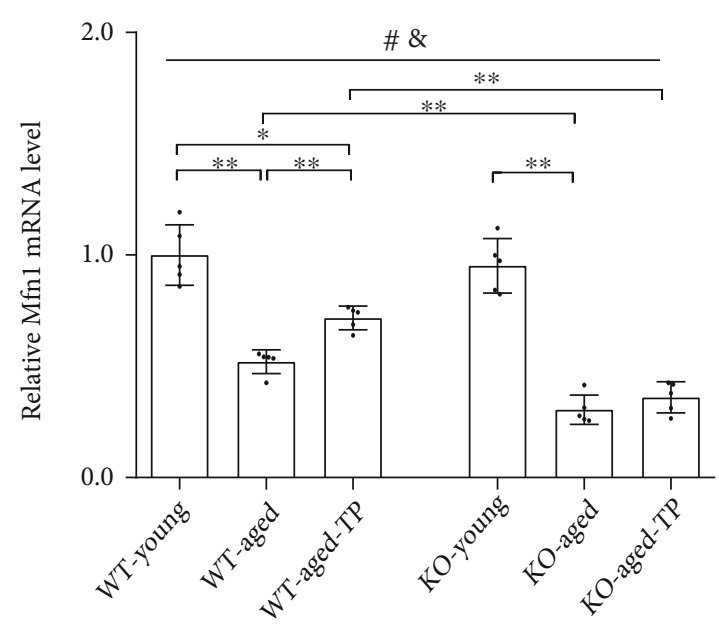

(b)

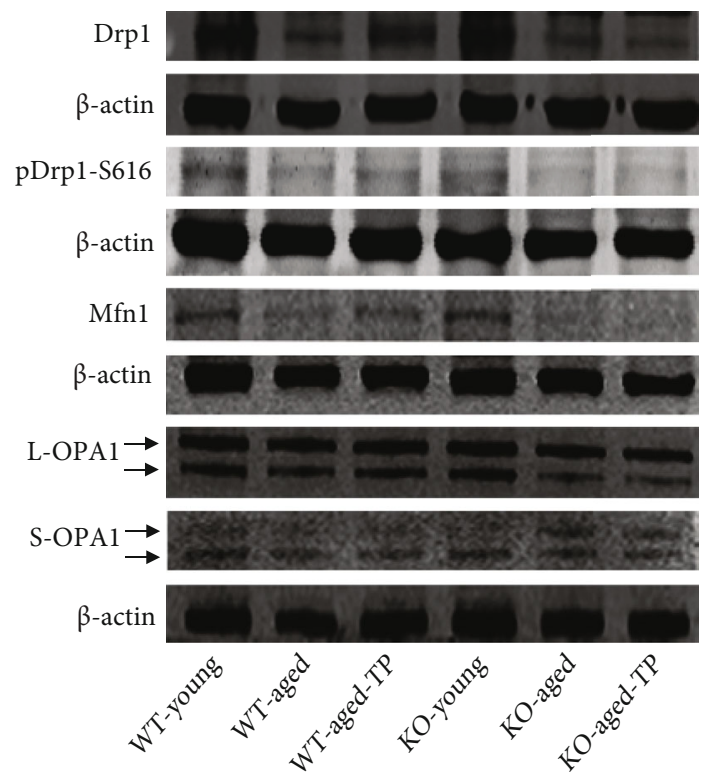

(d)

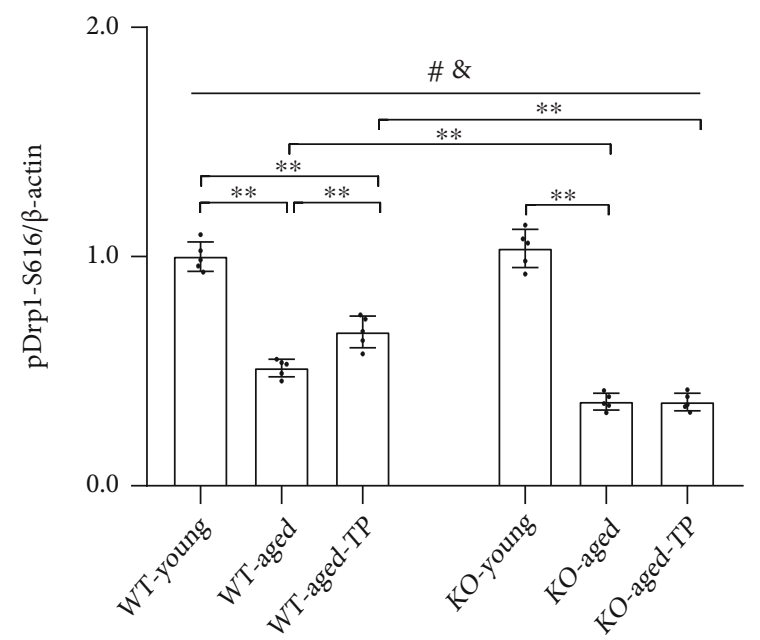

(f)

FIgURE 7: Continued. 


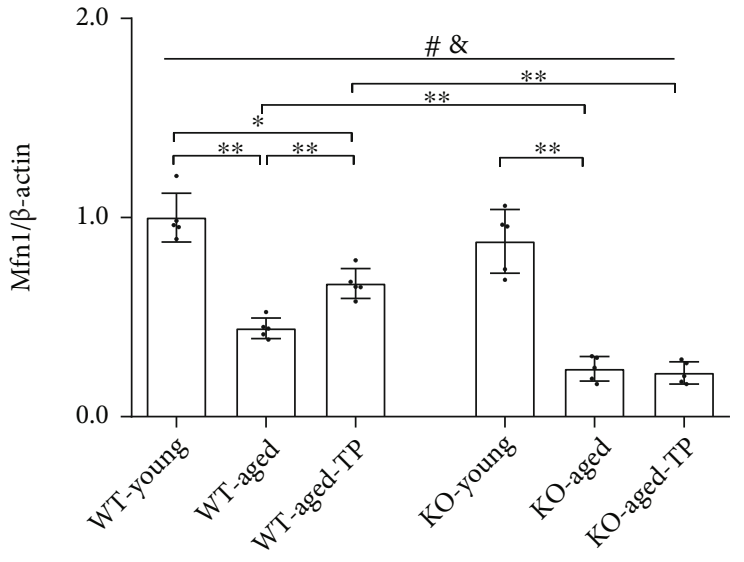

(g)

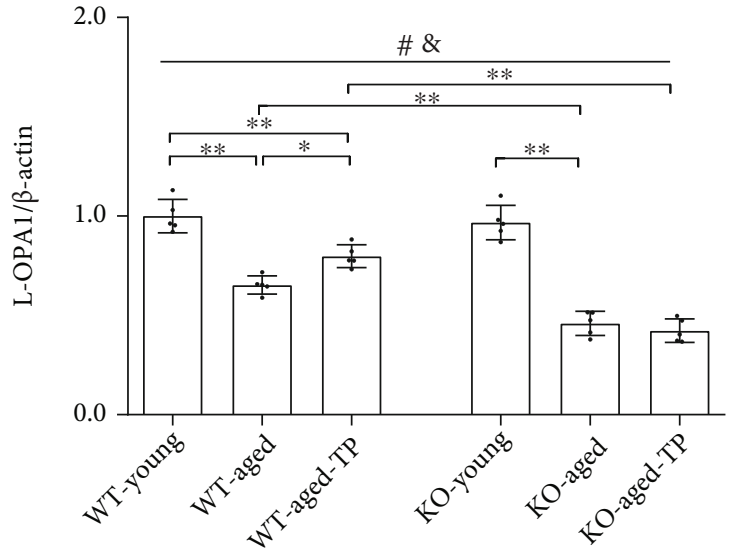

(h)

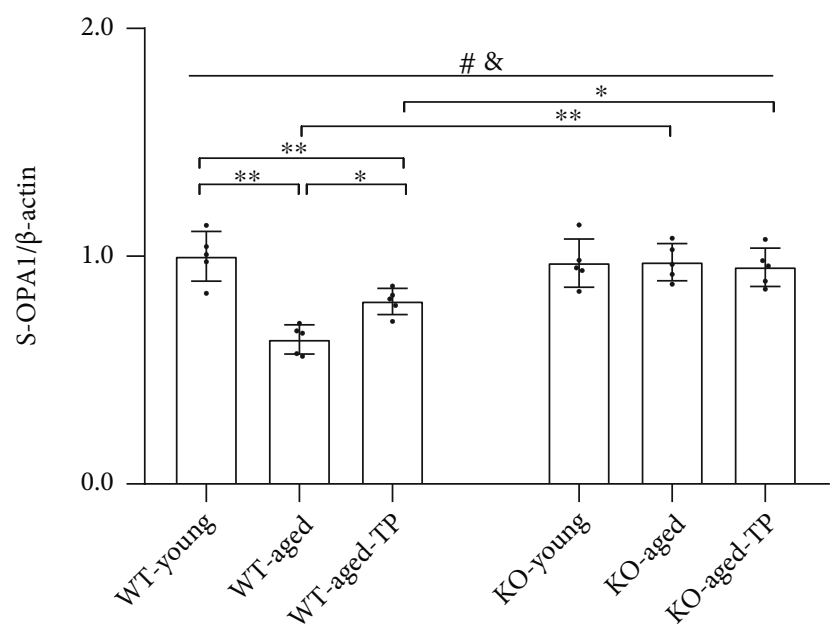

(i)

FIGURE 7: Effects of TP supplementation on mitochondrial dynamics in the substantia nigra of Nrf2 KO-aged male mice. (a) Drp1, (b) Mfn1, and (c) OPA1 mRNA were revealed by qPCR. (d, e) Drp1, (d, f) pDrp1-S616, (d, g) Mfn1, (d, h) L-OPA1, and (d, i) S-OPA1 proteins were detected by immunoblotting. Data were presented as mean $\pm \mathrm{SD} ; n=5 .{ }^{\#} P<0.05$ main effect of genotype by two-way ANOVA; ${ }^{\circledR} P<0.05$ main effect of treatment by two-way ANOVA. ${ }^{*} P<0.05$ and ${ }^{* *} P<0.01$.

in Experiment 1 were analyzed in the young experimental animal models of Experiment 2 (Figures 9-15). Orchiectomy to young mice of both genotypes weakened openfield activity $(P<0.01$, Figures $9(\mathrm{a})-9(\mathrm{e}))$. Gonadectomized young male mice of both genotypes showed the decreased nigrostriatal dopaminergic activity $(P<0.01$, Figures 10 (a)$10(\mathrm{f})$ ), the increased oxidative stress (GSH/GSSG, MDA, mitochondrial $\mathrm{H}_{2} \mathrm{O}_{2}$, and mitochondrial 3-NT, $P<0.01$, Figures 11(a)-11(e). 3-NT: AOD, $P<0.05$; number, $P<$ 0.01 , Figures $11(\mathrm{f})-11(\mathrm{~h})$ ), the reduced mitochondrial function (MMP: WT, $P<0.05$; KO, $P<0.01$, Figure 12(a). Mitochondrial ATP, mitochondrial complexes I, IV, and V, $P<0.01$, Figures 12(b), 12(c), 12(f), and 12(g)), and the decreased expression levels of PGC- $1 \alpha$, NRF-1, NRF-2, and TFAM $(P<0.01$, Figures 13(a)-13(l)), as well as the lowered mitochondrial content (CS, mtDNA/nDNA, $P<0.01$, Figures $14(\mathrm{a})$ and $14(\mathrm{~b})$ ) in the studied brain region. The increased Drp1 and pDrp1-S616, as well as the reduced Mfn1, L-OPA1, and S-OPA1 levels, were found in the SN of gonadectomized young male mice of both genotypes $(P<0.01$, Figures 15(a)-15(i)). KO-GDX mice presented the aggravated effects of orchiectomy on the above parameters compared with WT-GDX mice, except for climbing (Figure 9(c)), 3-NT (AOD and number of 3-NT-ir cells, Figures 11(f)-11(h)), ATP (Figure 12(b)), PGC-1 $\alpha$ (Figures 13(a), 13(e), and 13(i)), NRF-1 (Figures 13(b), 13(f), and 13(j)), TFAM (Figures 13(d), 13(h), and 13(l)), Mfn1 (Figures 15(b), 15(d), and 15(g)), OPA1 mRNA (Figure 15(c)), L-OPA1 (Figures 15(d) and 15(h)), and S-OPA1 (Figures 15(d) an(d) 15(i)). Supplementation with TP restored the observed parameters above in WT-GDX mice to WT-sham level except for DOPAC $(P<0.05$, Figure 10(e)). The above parameters in KO-GDX-TP mice were ameliorated except for HVA (Figure 10(f)) and 3-NT (AOD and number of 3-NT-ir cells, Figures 11(f)-11(h)) relative to KO-GDX mice, but not reversed to KO-sham level except for NRF-2 (Figures 13(c), 13(g), and 13(k)). NRF-2 level in the SN of KO-GDX-TP mice reached the level of KO-sham mice. 


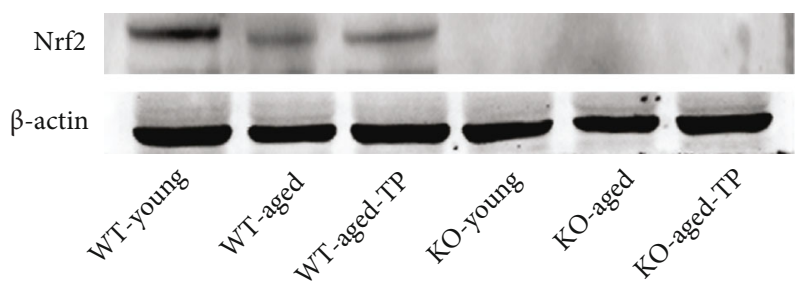

(a)

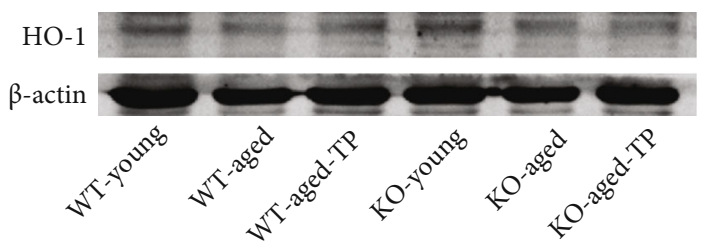

(c)

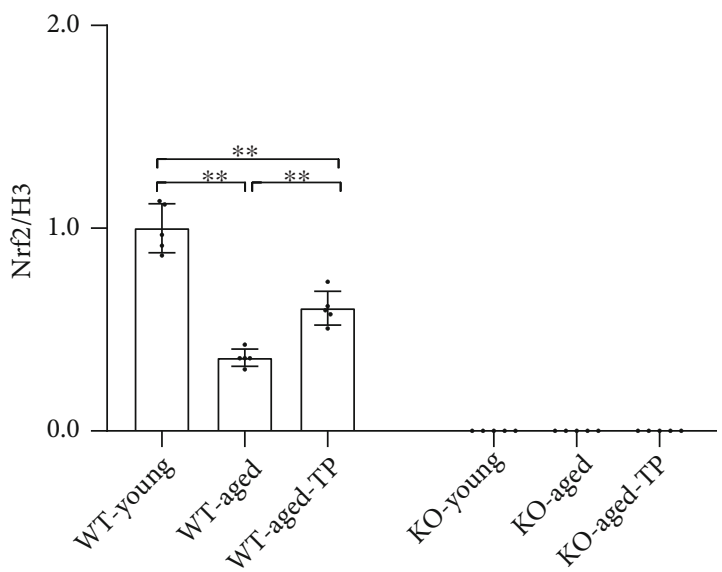

(e)

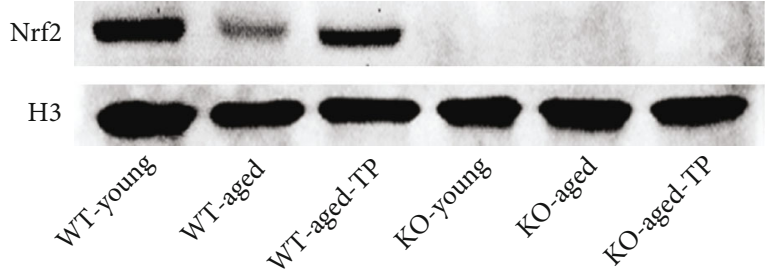

(b)

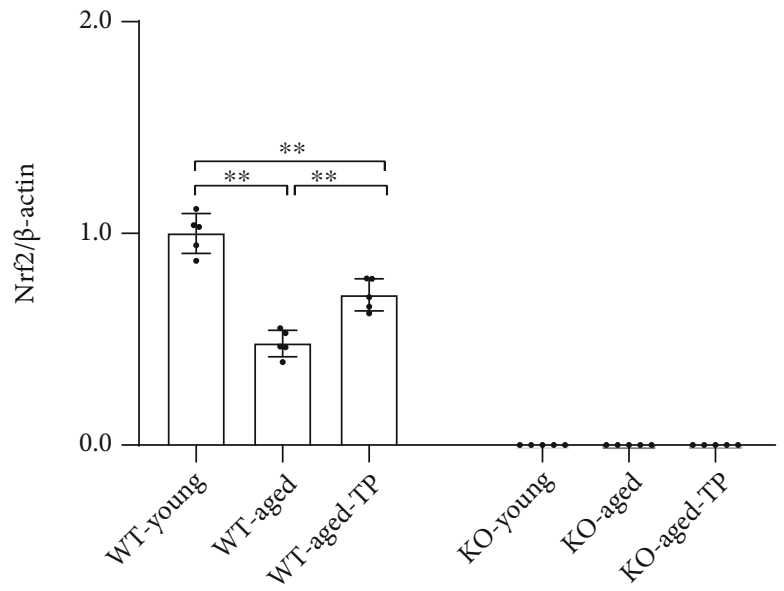

(d)

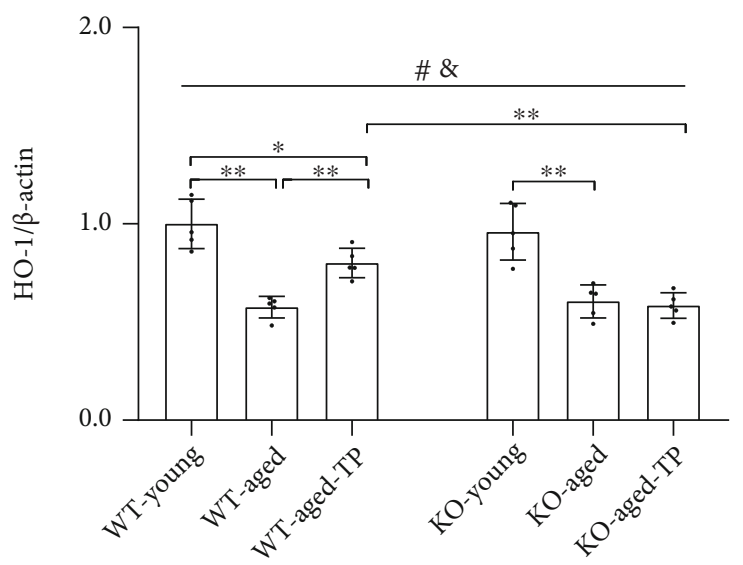

(f)

Figure 8: Effects of TP supplementation on Nrf2 in the substantia nigra of aged male mice. (a, d) Nrf2 in the SN, (b, e) Nrf2 in nucleus fraction, and (c, f) HO-1 protein in the $\mathrm{SN}$ were detected by immunoblotting. Data were presented as mean $\pm \mathrm{SD} ; n=5$. ${ }^{\#} P<0.05$ main effect of genotype by two-way ANOVA; ${ }^{\circledR} P<0.05$ main effect of treatment by two-way ANOVA. ${ }^{*} P<0.05$ and ${ }^{* *} P<0.01$.

\section{Discussion}

In this study, we demonstrated that WT-aged male mice presented uncoordinated walking gait, as well as declined open-field activity, nigrostriatal dopaminergic activity, oxidative balance, and mitochondrial function, as well as downregulated mitochondrial biogenesis and mitochondrial dynamics. Nrf2 deficiency exacerbated the deficits of the above parameters in aged male mice. Supplementation with testosterone to WT-aged male mice significantly ameliorated open-field activity, walking gait, nigrostriatal dopaminergic activity, oxidative balance, and mitochondrial function and upregulated mitochondrial biogenesis and mitochondrial dynamics. However, the above effects of testosterone on WT-aged male mice were not shown in Nrf2 KO-aged male mice. Orchidectomy to young male mice decreased mitochondrial function, downregulated mitochondrial biogenesis, and altered mitochondrial dynamics balance. Supplementation with testosterone to Nrf2 KOGDX mice only ameliorated the alterations above but did not reverse them to sham level. Thus, Nrf2 plays an important role in ameliorating open-field activity, walking gait, and nigrostriatal dopaminergic activity of aged male mice by testosterone supplementation, which is related to the activated Nrf2 by TP. Nrf2 deficiency attenuated the efficiency of testosterone supplementation in improving 


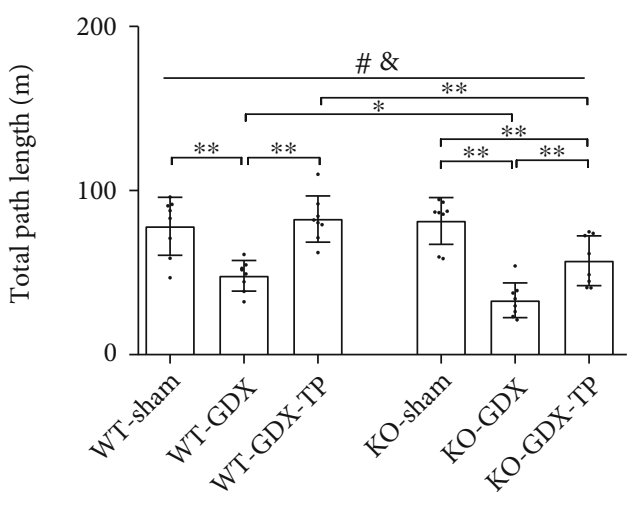

(a)

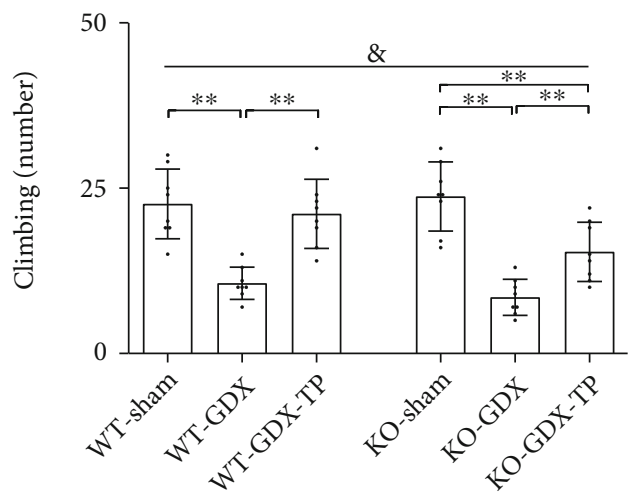

(c)

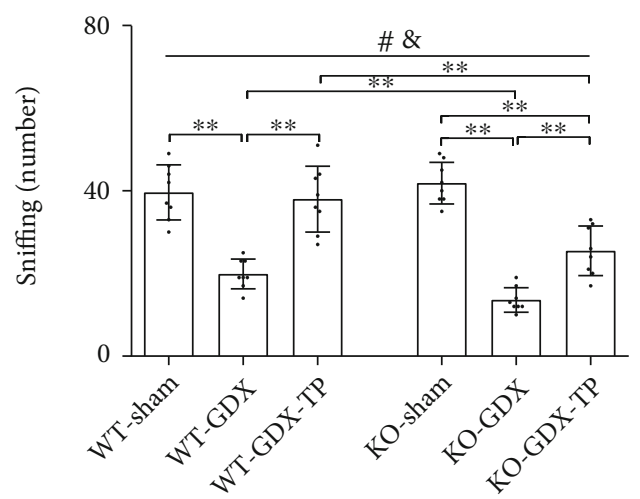

(e)

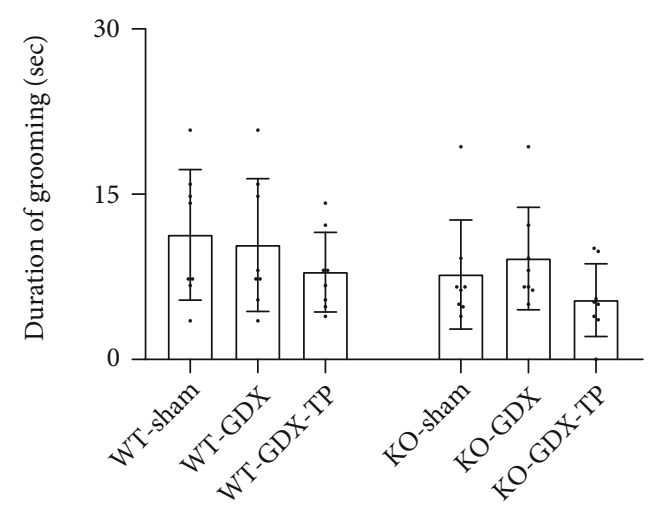

(g)

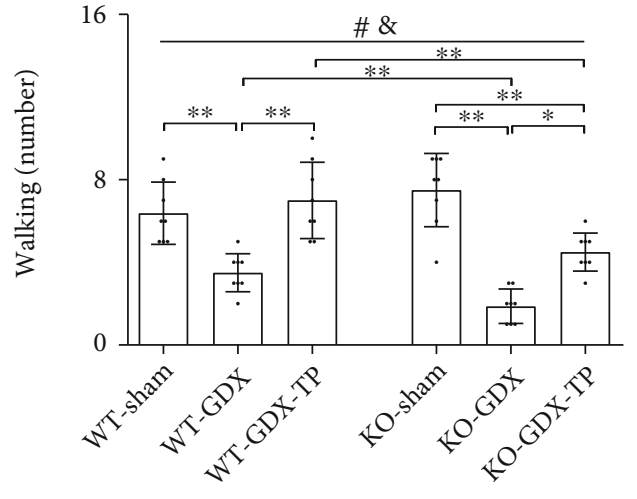

(b)

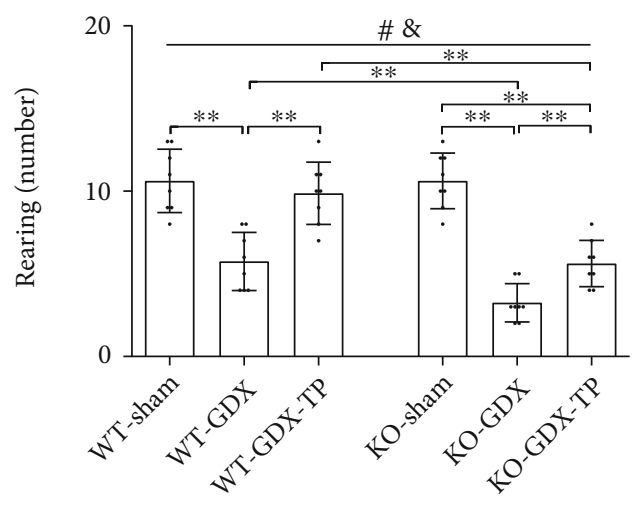

(d)

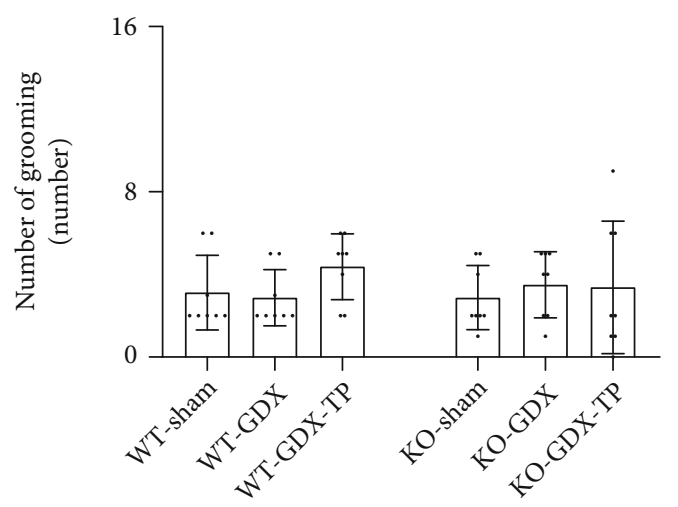

(f)

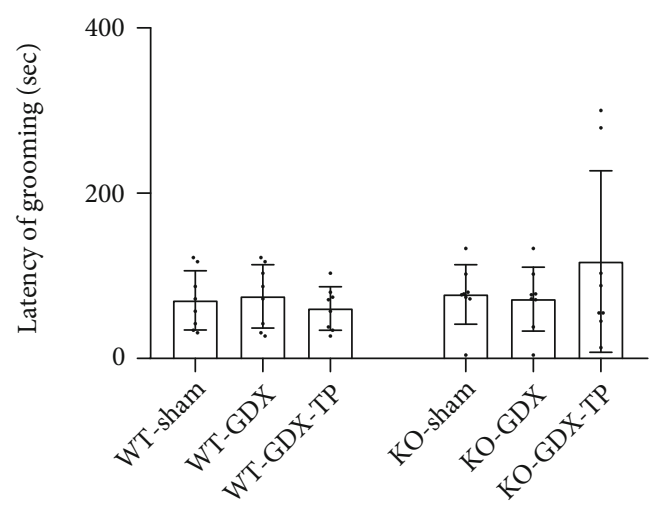

(h)

Figure 9: Continued. 


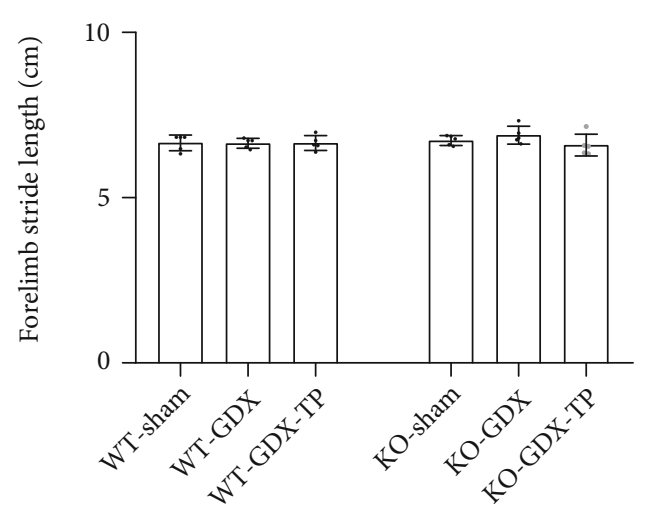

(i)

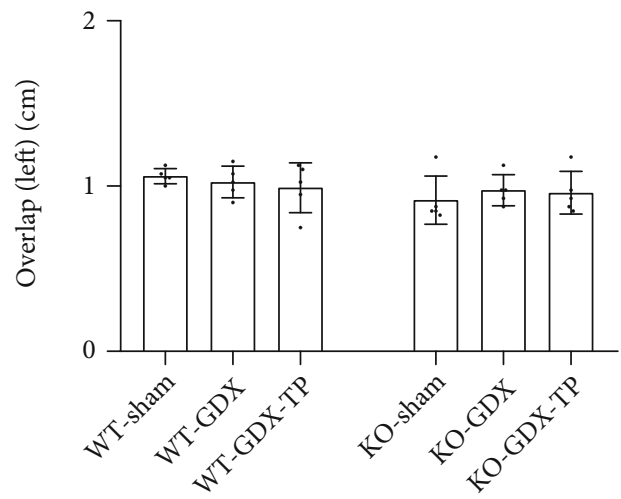

$(\mathrm{k})$

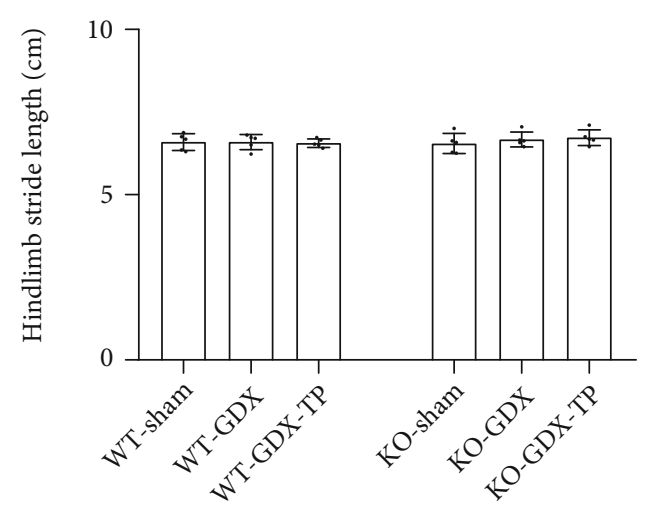

(j)

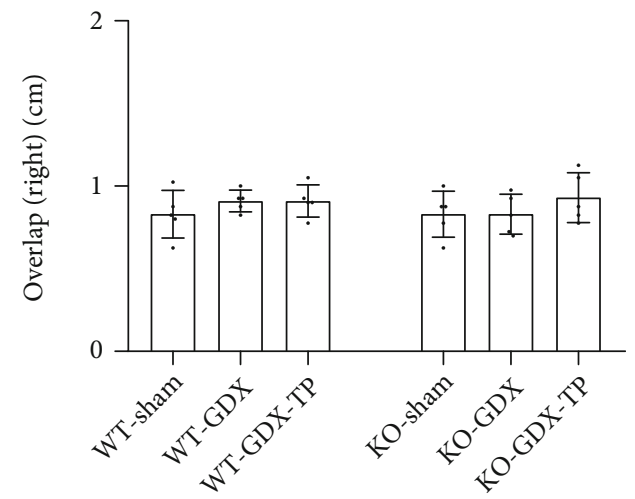

(1)

FIGURE 9: Effects of TP supplementation on open-field activity and walking gait of Nrf2 KO GDX male mice: (a) total path length, (b) walking, (c) climbing, (d) rearing, (e) sniffing, (f) number of grooming, (g) duration of grooming, (h) latency of grooming, (i) forelimb stride length, $(\mathrm{j})$ hindlimb stride length, $(\mathrm{k})$ overlap of left footprints, and (l) overlap of right footprints. Data were presented as mean \pm SD; $n=8$ for open-field test; $n=5$ for footprint test. ${ }^{\#} P<0.05$ main effect of genotype by two-way ANOVA; ${ }^{\circledR} P<0.05$ main effect of treatment by two-way ANOVA. ${ }^{*} P<0.05$ and ${ }^{* *} P<0.01$.

mitochondrial function of the substantia nigra in aged male mice via mitochondrial biogenesis and mitochondrial dynamics to some extent.

Motor activity gradually declines during the aging process [29], and this decline is related to the age-related decline of nigral neuronal function [29, 31]. A persistent loss of nigrostriatal neurons results in severe locomotor impairment, such as altered gait pattern [32]. Previous studies demonstrated that androgen influences brain function to induce behavioral alterations [14, 33]. Androgen supplementation increases motor and exploratory behaviors of aged male rodents $[14,18,33]$ and ameliorated motor symptoms of men with PD to some extent [34]. The amelioration of motor and exploratory behaviors in testosteronesupplemented aged animals is related to nigrostriatal dopaminergic activity enhanced by androgen $[14,15]$. The present study showed that supplementation with testosterone significantly improved motor and exploratory behaviors, as well as walking gait of WT-aged male mice and enhanced nigrostriatal dopaminergic activity. However, the above effects of testosterone supplementation on WT-aged male mice were not found in Nrf2 KO-aged male mice. The administration of testosterone to $\mathrm{Nrf} 2 \mathrm{KO}$-aged male mice neither improved motor and exploratory behaviors as well as walking gait nor enhanced nigrostriatal dopaminergic activity, which demonstrated that Nrf2 might be required for testosterone to ameliorate their neurochemical defects of the nigrostriatal dopaminergic system in aged male mice. $\mathrm{Nrf} 2$ is widely expressed in the central nervous system including the SN. As a master transcription factor, Nrf2 binds ARE in the promoters of Nrf2 target genes [9, 35]. When it is activated, a series of cytoprotective enzymes and antioxidants are induced [35], such as HO-1. Nrf2 with its downstream gene products constitutes the main antioxidant defense system to degrade free radicals $[9,35]$. The previous studies showed that Nrf2 protects nigrostriatal dopaminergic neurons and maintains their normal activity [9]. In PD, remaining dopaminergic neurons exhibit stronger nuclear Nrf2 immunoreactive staining [36]. Thus, our results above indicated that Nrf2 might determine the ameliorative effects of testosterone supplementation on the nigrostriatal dopaminergic system of aged male mice.

How does testosterone exerts its ameliorative effects on the nigrostriatal dopaminergic system of aged male animals has not been elucidated. Improved mitochondrial function might explain the ameliorative effects of TP supplementation on the nigrostriatal dopaminergic system during aging process by modulating oxidative stress, mitochondrial 


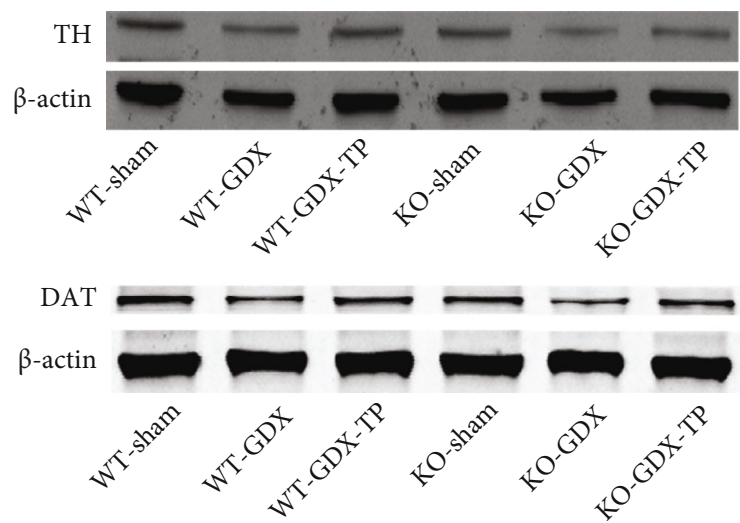

(a)

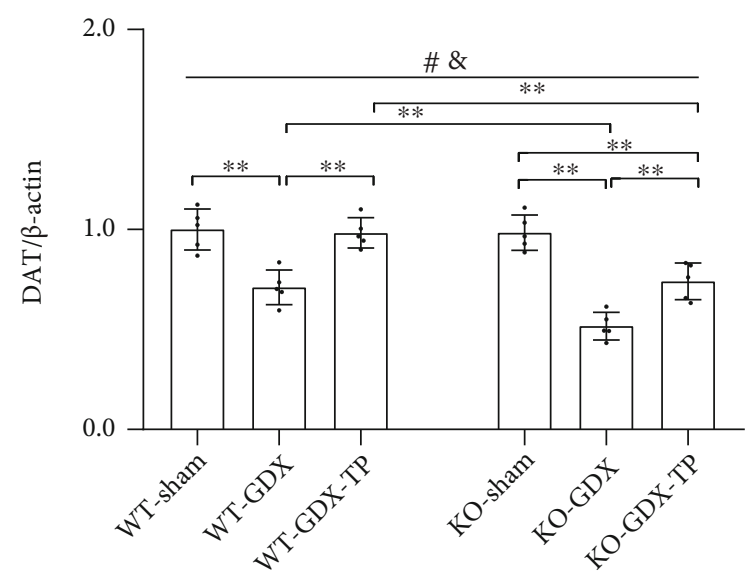

(c)

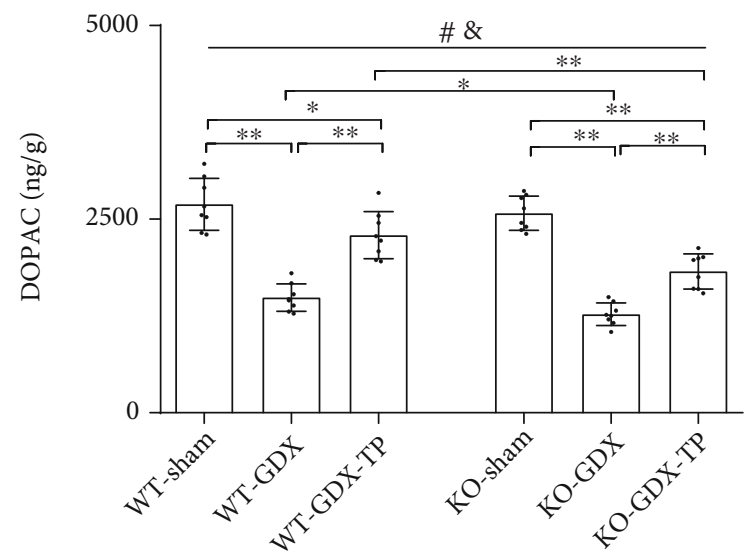

(e)

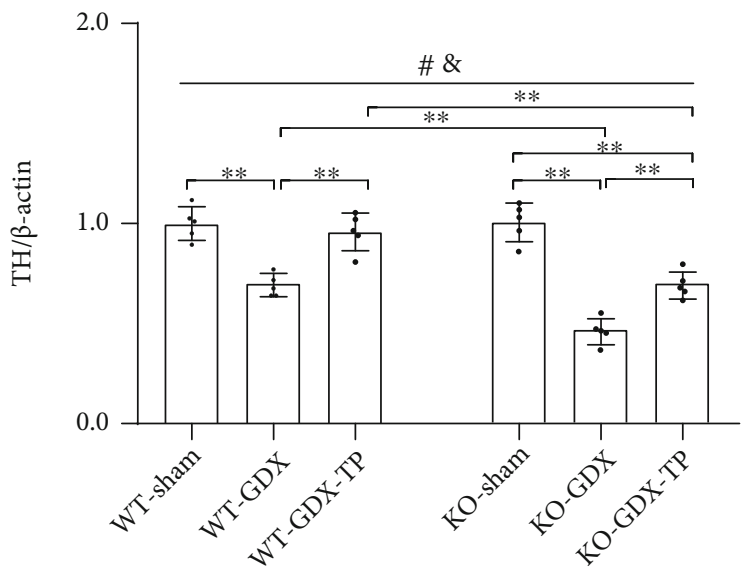

(b)

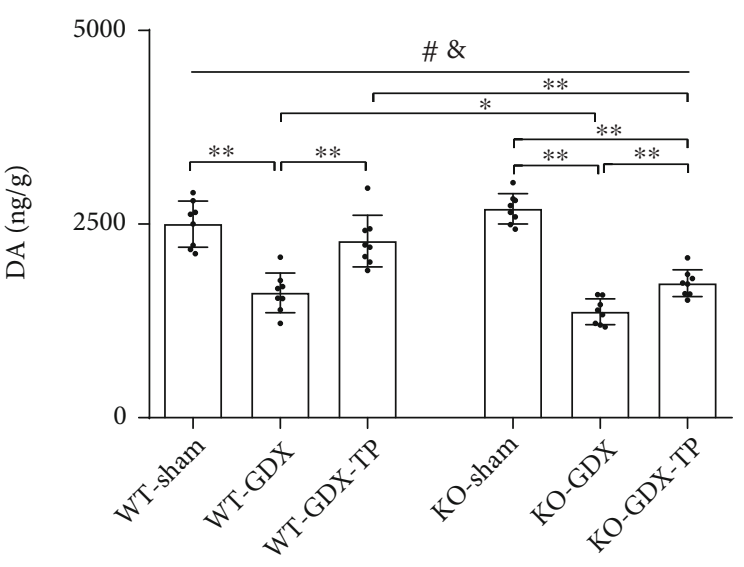

(d)

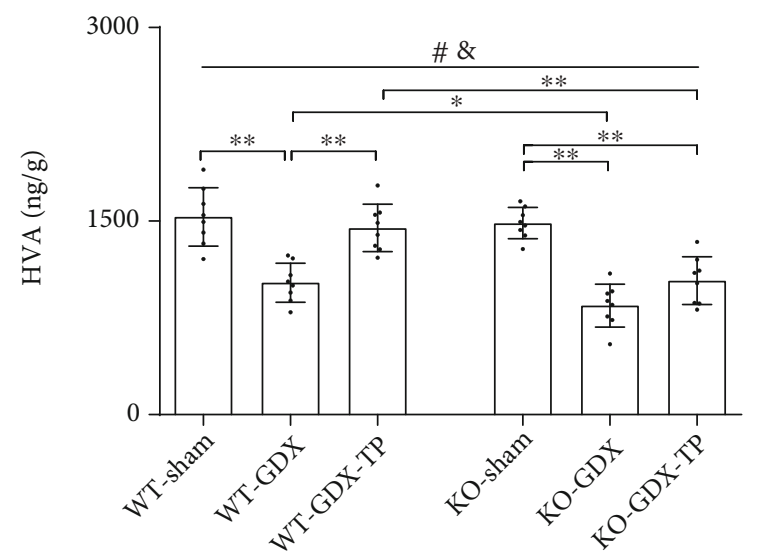

(f)

FIGURE 10: Effects of TP supplementation on dopaminergic activity in the caudate putamen of Nrf2 KO GDX male mice. (a, b) TH and (a, c) DAT were detected by immunoblotting. (d) DA, (e) DOPAC, and (f) HVA were measured by LC-MS/MS assay. Data were presented as mean \pm SD; $n=5$ for TH and DAT; $n=8$ for DA, DOPAC, and HVA. ${ }^{\#} P<0.05$ main effect of genotype by two-way ANOVA; ${ }^{\circledR} P<0.05$ main effect of treatment by two-way ANOVA. ${ }^{*} P<0.05$ and ${ }^{* *} P<0.01$.

biogenesis, and mitochondrial dynamics. Oxidative stress is characterized by an unbalance between overproduced ROS and antioxidant defenses in cells. It is one of the major factors in aging and in aging-related neurodegenerative diseases [37]. Under physiological conditions, cellular ROS are eradicated by the endogenous antioxidative defense systems.
However, under pathological circumstances, mitochondrial dysfunction, such as reduced MMP (an important indicator of mitochondrial function) and impaired oxidative phosphorylation, produces excessive ROS, resulting in oxidative damage to cells by targeting proteins, lipids, or DNA [2]. The mitochondrion is a main organelle producing 


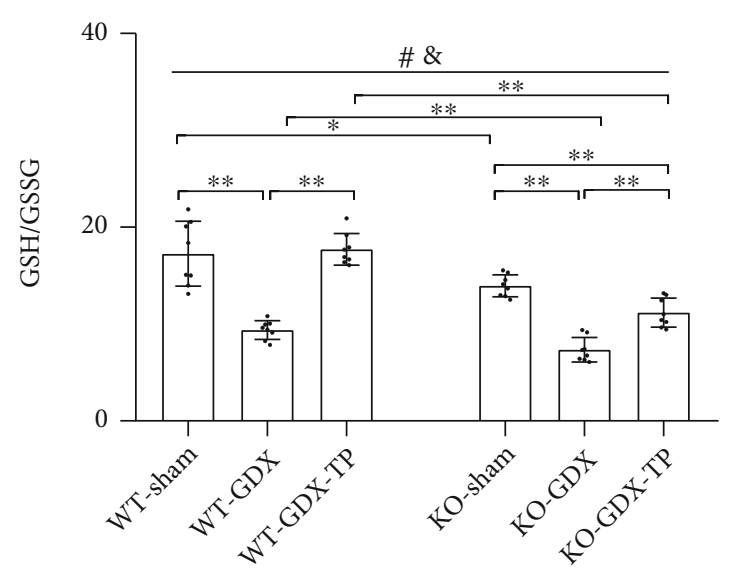

(a)

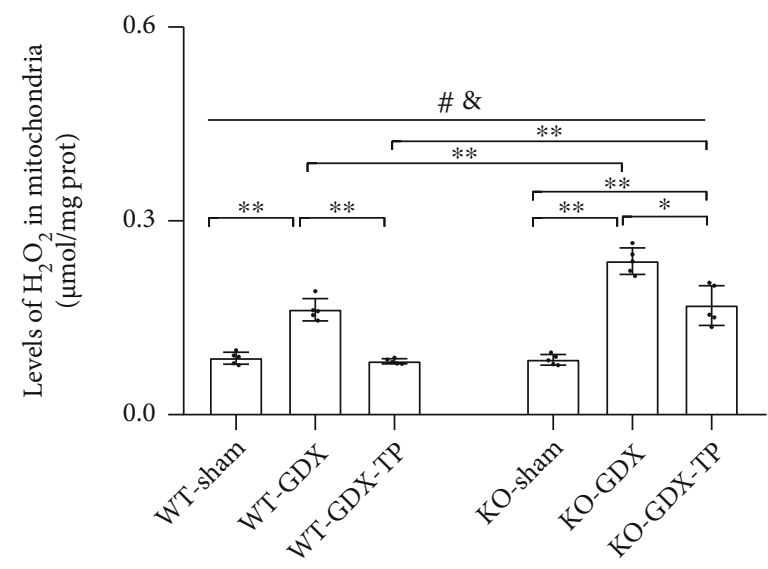

(c)

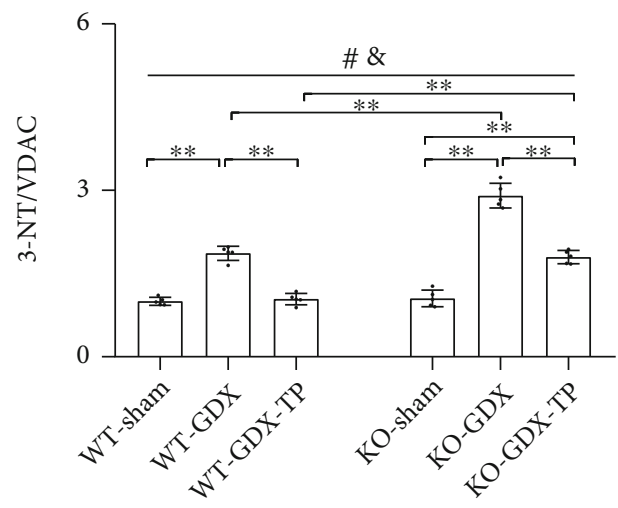

(e)

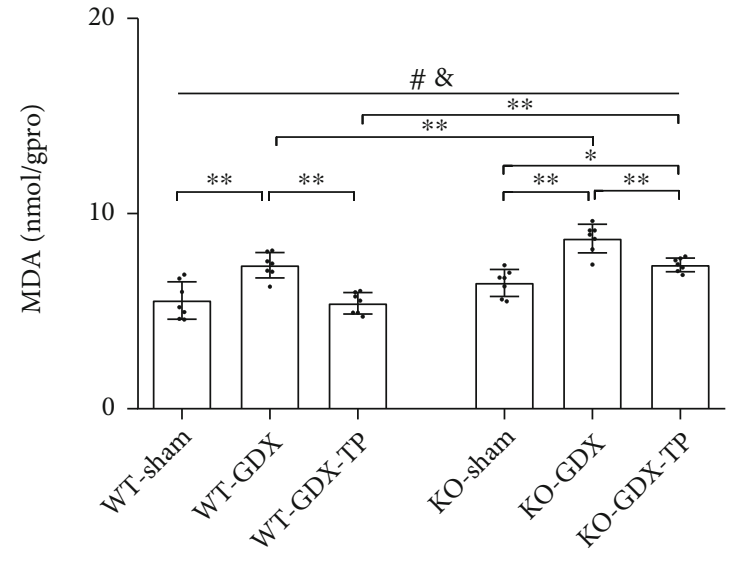

(b)

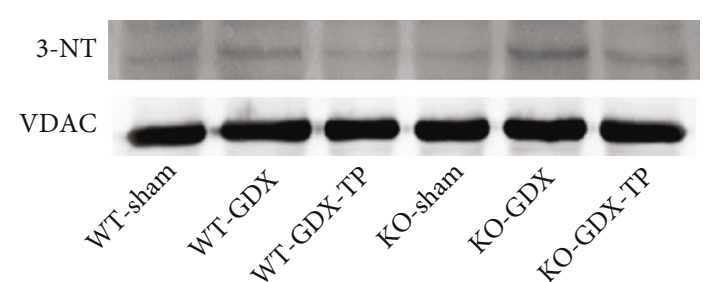

(d)

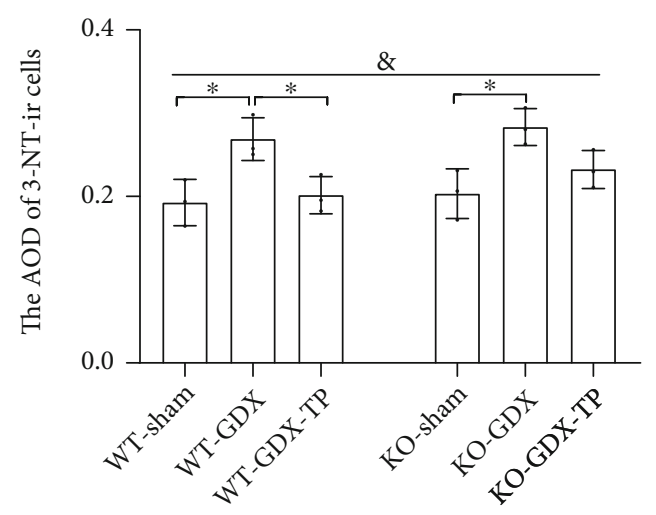

(f)

Figure 11: Continued. 


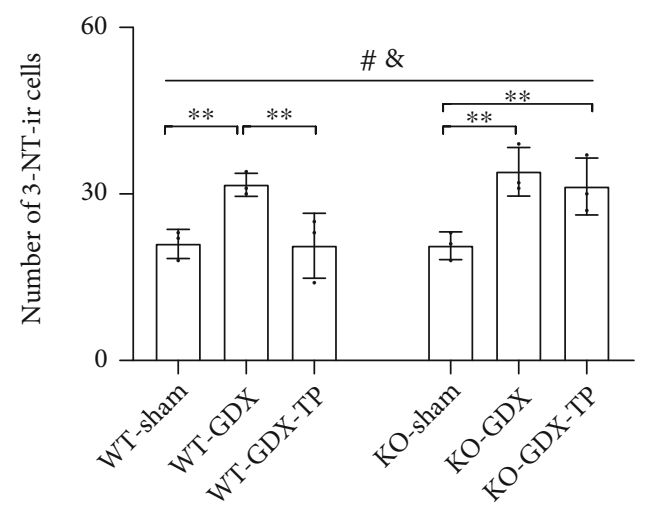

(g)
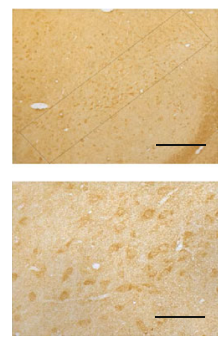

WT-sham
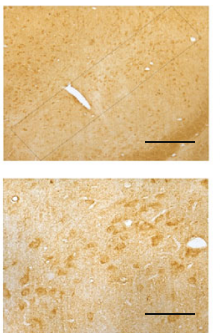

WT-GDX

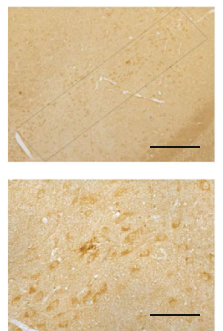

WT-GDX-TP

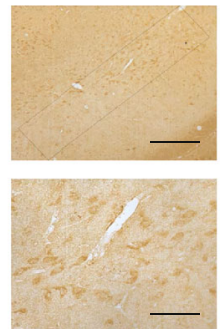

KO-sham

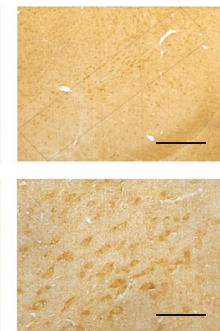

KO-GDX

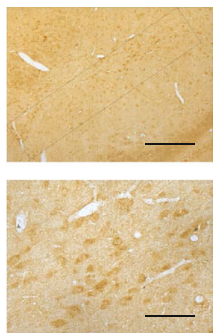

KO-GDX-TP

(h)

FIgURE 11: Effects of TP supplementation on oxidative balance in the substantia nigra of Nrf2 KO GDX male mice. (a) GSH/GSSG, (b) MDA, and (c) mitochondrial $\mathrm{H}_{2} \mathrm{O}_{2}$ were assessed by spectrophotometry. (d, e) Mitochondrial 3-NT was measured by immunoblotting; $(\mathrm{f}-\mathrm{h}) 3-\mathrm{NT}$ in the SN was detected by immunohistochemistry. Data were presented as mean $\pm \mathrm{SD} ; n=8$ for GSH/GSSG; $n=7$ for MDA; $n=5$ for mitochondrial 3-NT; $n=3$ for 3 -NT immunohistochemistry. Scale bars $=50 \mu \mathrm{m}$ (lower panel); scale bars $=200 \mu \mathrm{m}$ (upper panel). ${ }^{\#} P<0.05$ main effect of genotype by two-way ANOVA; ${ }^{8} P<0.05$ main effect of treatment by two-way ANOVA. ${ }^{*} P<0.05$ and ${ }^{* *} P<0.01$

superoxide anion $\left(\mathrm{O}_{2}{ }^{-}\right)$. It is involved in oxidative damage to macromolecules and can be converted to $\mathrm{O}_{2}$ and $\mathrm{H}_{2} \mathrm{O}_{2}$. $\mathrm{GSH}$ and GSSG are two different forms of glutathione. Glutathione peroxidase catalyzes the reduction of $\mathrm{H}_{2} \mathrm{O}_{2}$ via $\mathrm{GSH}$ to produce GSSG and $\mathrm{H}_{2} \mathrm{O}$. MDA is an important indicator for detecting lipid peroxidation of biological membranes. It is the peroxidation product of phospholipids or lipoproteins of cytoplasmic membranes [38]. 3-NT is another biomarker of oxidative stress to predict the level of oxidative damage $[39,40]$. It is formed due to nitration of protein-bound and free tyrosine residues by reactive peroxynitrite molecules. The previous study showed that the levels of Nrf2 and its downstream gene products, such as NQO-1 and HO-1, are higher in TP-treated aged male rats than in their counterpart control [15]. TP-treated aged male rats show decreased MDA and GSH/GSSG in the SN compared with control $[15,18]$. In the present study, decreased GSH/GSSG and increased MDA as well as mitochondrial $\mathrm{H}_{2} \mathrm{O}_{2}$ and 3NT revealed the existed oxidative damage in the $\mathrm{SN}$ of aged male mice, especially in Nrf2 KO-aged male mice. Decreased MMP and mitochondrial ATP levels, as well as activities of mitochondrial complexes I, IV, and V, indicated mitochondrial dysfunction in the SN of aged male mice. Testosterone supplementation decreased MDA, as well as mitochondrial $\mathrm{H}_{2} \mathrm{O}_{2}$ and 3-NT levels, and increased GSH/GSSG ratio, MMP, mitochondrial ATP content, and activities of mitochondrial complexes I, IV, and V in the SN of WT-aged male mice. The results above showed improved mitochondrial function in WT TP-treated aged male mice. By comparing the results of 3-NT immunohistochemistry with those of 3-NT immunoblotting, it was found that although both methods detected a significant increase in 3-NT levels in the SN of WT-aged male mice before TP treatment, immunohistochemistry-revealed 3-NT levels only showed decreased trend in WT-aged-TP mice, not reaching significance. However, immunoblotting detected significantly decreased 3-NT levels in WT-aged-TP mice. The former observed the cells in the $\mathrm{SN}$, while the latter located isolated mitochondria from the SN, which will more accurately reflect the subtle changes in damaged organelles. Mitochondria, as primary sources of ROS, were damaged in WT-aged male mice. The mitochondrial ultrastructure by an electron microscope supported the finding above. WT-aged mice showed ultrastructural abnormalities of mitochondria in a way, such as unclear cristae, which was improved by TP supplementation. However, improved oxidative balance status and mitochondrial function were only shown in the SN of TP-treated WT-aged male mice, not in TP-treated Nrf2 KO-aged male mice. It was suggested that Nrf2 deficiency blocked the efficiency of testosterone replacement in ameliorating mitochondrial function in the $\mathrm{SN}$ of aged male mice, leading to oxidative damage to $\mathrm{SN}$.

Aging and aging-related neurodegenerative disorders are often accompanied by mitochondrial dysfunction [21]. 


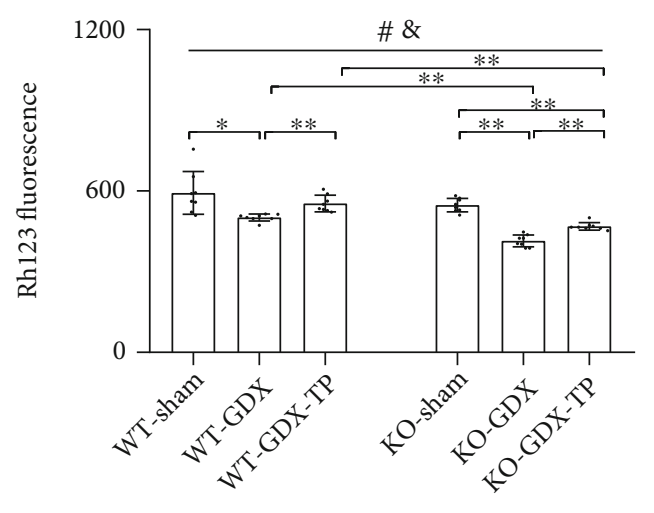

(a)

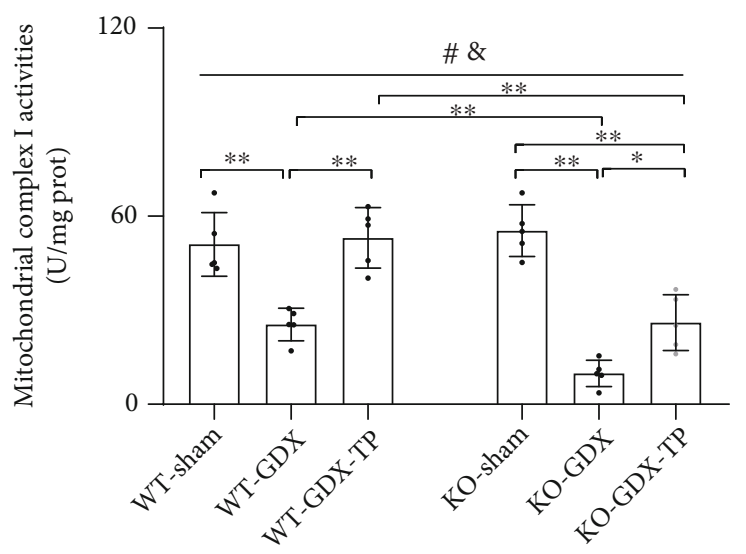

(c)

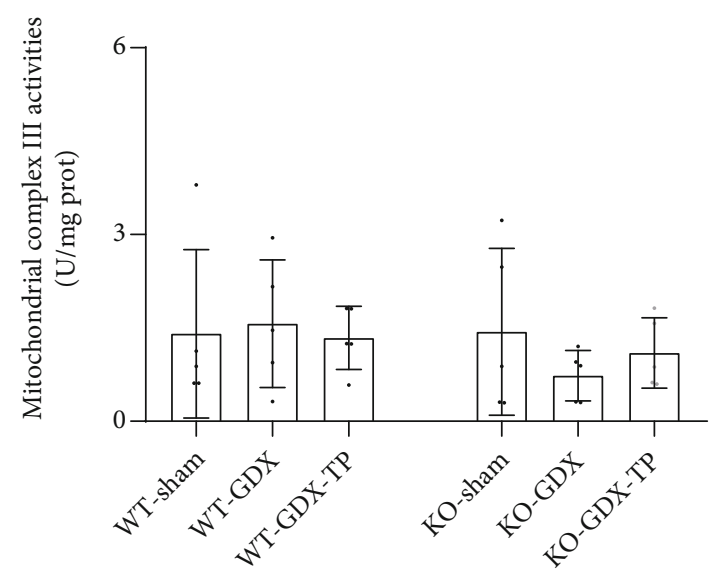

(e)

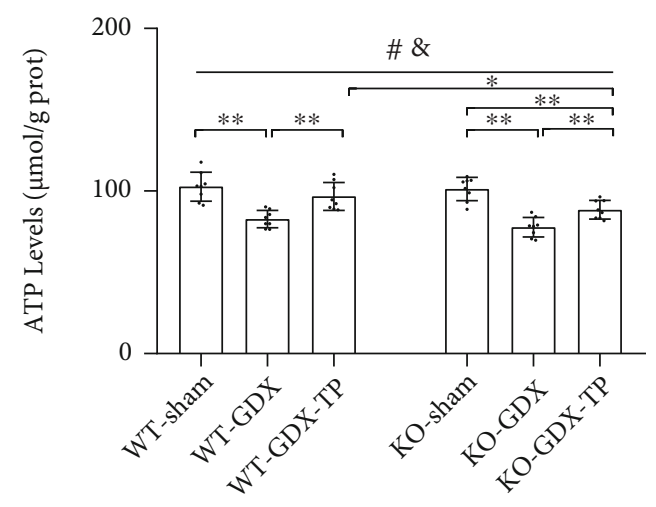

(b)

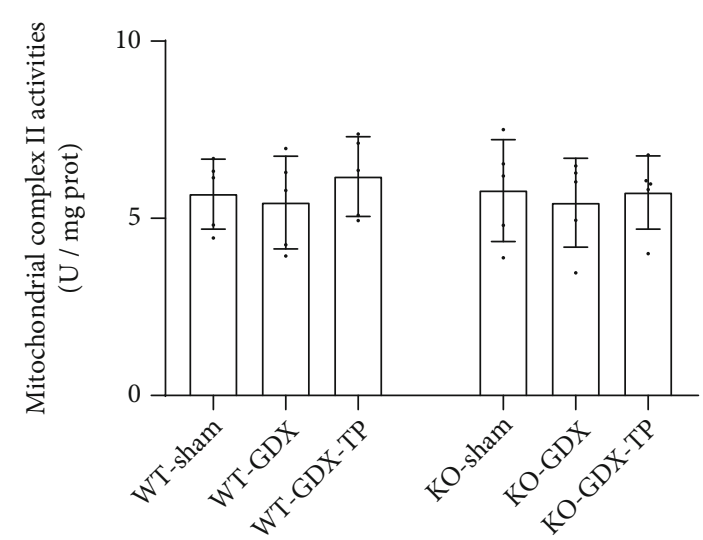

(d)

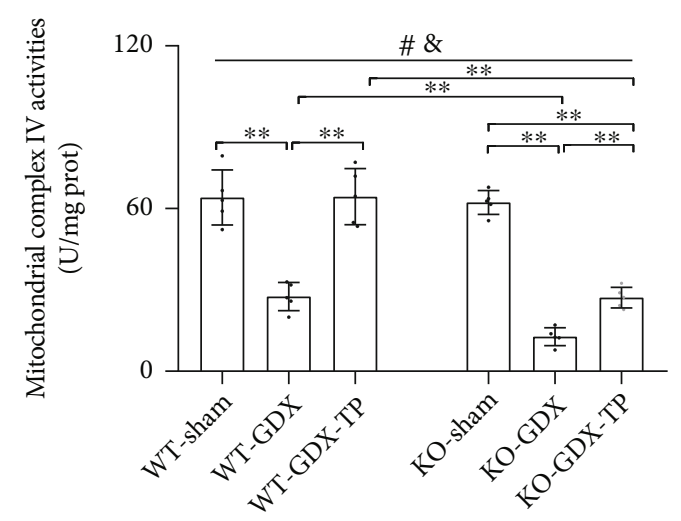

(f)

Figure 12: Continued. 


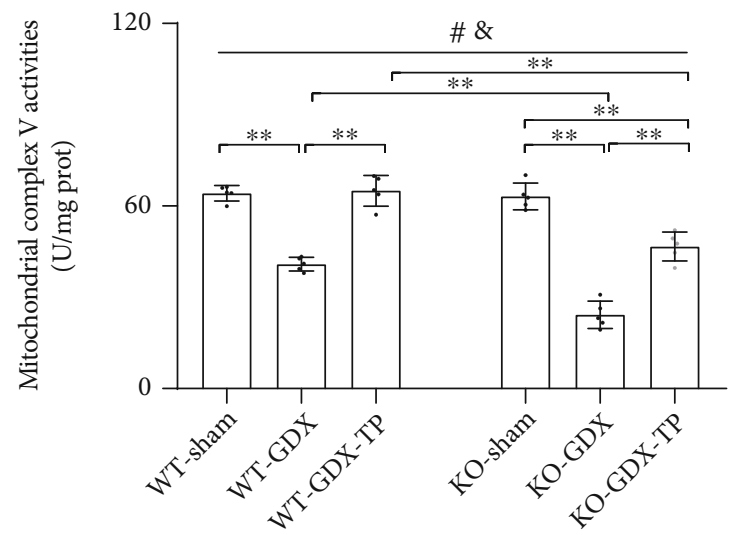

(g)

FIGURE 12: Effects of TP supplementation on mitochondrial function in the substantia nigra of Nrf2 KO GDX male mice. (a) Mitochondrial membrane potential was revealed using the Rh123 fluorescence method. (b) Mitochondrial ATP levels, (c) mitochondrial complex I activity, (d) mitochondrial complex II activity, (e) mitochondrial complex III activity, (f) mitochondrial complex IV activity, and (g) mitochondrial complex V activity were revealed by spectrophotometry. Data were presented as mean $\pm \mathrm{SD} ; n=8$ for MMP and mitochondrial ATP levels; $n=5$ for mitochondrial complexes I-V. ${ }^{*} P<0.05$ main effect of genotype by two-way ANOVA; ${ }^{\circledR} P<0.05$ main effect of treatment by twoway ANOVA. ${ }^{*} P<0.05$ and ${ }^{* *} P<0.01$.

During aging process, promoting mitochondrial biogenesis might be a cellular strategy to maintain normal mitochondrial function [6]. Mitochondrial biogenesis is mainly regulated by PGC- $1 \alpha$, a key regulator of mitochondrial biogenesis and antioxidative defense $[8,41]$. It controls the rate of the mitochondrial biogenesis through its downstream targets, NRF-1, NRF-2, and TFAM [41, 42]. In vitro study showed that testosterone increases PGC- $1 \alpha$, NRF-1, and TFAM at transcription level in $\mathrm{C}_{2} \mathrm{C}_{12}$ myotubes $[43,44]$ and flutamide (androgen receptor antagonist) reduces the testosterone-induced upregulation of PGC-1 $\alpha$ [44], NRF-1, and TFAM [43]. Moreover, testosterone deficiency caused by orchiectomy decreases PGC- $1 \alpha$, NRF-1, and TFAM gene expression in the adult rat hippocampus and supplementation of testosterone to orchiectomy adult rats restores them in the hippocampus to control levels [19]. Similarly, we found in the present study that orchiectomy reduced PGC$1 \alpha$, NRF-1, NRF-2, and TFAM gene expression in the SN of WT-young male mice. Reduced PGC- $1 \alpha$, NRF-1, NRF2 , and TFAM gene expression, with decreased CS activity and mtDNA copy number in the SN of GDX WT-young male mice, was reversed to sham levels by TP supplementation, which suggested that mitochondrial biogenesis in the $\mathrm{SN}$ is androgen-related. Mitochondrial biogenesis has been found to decline in the skeletal muscle and in the brain during aging [18, 45]. During the aging process, PGC- $1 \alpha$ expression level declines in the skeletal muscle as well as in the brain and this can be reversed in the skeletal muscles by exercise training of aged male rats [46] and ameliorated in the brain by $\mathrm{TP}$ supplementation to aged male rats [18], which demonstrated the aging skeletal muscle cells and neurons still remain the ability to upregulate mitochondrial biogenesis through increasing PGC- $1 \alpha$ expression $[18,46]$. Narasimhan et al. found that the PGC- $1 \alpha$ protein levels were decreased in the skeletal muscle of Nrf2 KO-aged mice compared with WT-aged mice [47]. In the present study, we found that PGC- $1 \alpha$ and its downstream target expression levels in the SN were significantly reduced in WT-aged mice and Nrf2 KO further decreased their expression levels in the $\mathrm{SN}$ upon aging. In addition, CS activity, mtDNA copy number, and mitochondrial number were significantly reduced in the $\mathrm{SN}$ of KO-aged mice due to Nrf2 deficiency. mtDNA copy number is frequently used as an index of mitochondrial content. CS activity is a more accurate indicator for mitochondrial content in tissues than mtDNA copy number [48]. Mitochondrial number in cells by electron microscopy directly displays the status of mitochondrial biogenesis and mitochondrial content. Supplementation with TP to WTaged mice increased expression of PGC- $1 \alpha$ and its downstream targets, as well as CS activity, mtDNA copy number, and mitochondrial number in the SN, and such effects of TP supplementation on them in the $\mathrm{SN}$ were blocked in $\mathrm{Nrf} 2$ KO-aged mice due to Nrf2 deficiency. A previous in vitro study revealed that silencing the PGC- $1 \alpha$ gene in SH-SY5Y cells results in the reduction of MMP and intracellular ATP content as well as the elevation of intracellular $\mathrm{H}_{2} \mathrm{O}_{2}$ generation [49]. Thus, the improvement of mitochondrial function (i.e., increased MMP, mitochondrial ATP, and complex activities, as well as decreased MDA, mitochondrial $\mathrm{H}_{2} \mathrm{O}_{2}$, and 3-NT and increased GSH/GSSG) in the SN of TP-treated WT-aged mice was associated with the TPpromoted mitochondrial biogenesis in large extent. Nrf2 played an important role in the effects of testosterone supplementation on the $\mathrm{SN}$ mitochondrial biogenesis of aged male mice.

The balanced mitochondrial dynamics is another important factor for improving mitochondrial function in the maintenance of normal cell activity [50-52]. Mitochondria undergo continuous rounds of fusion and fission in their life and form into dynamic networks within cells. Balanced mitochondrial dynamics are regulated by the GTPase family of proteins including Drp1, Mfn1/Mfn2, and OPA1 [53-55]. Drp1 is responsible for the mitochondrial fission [53]. Drp1 activity is regulated by its phosphorylation. Phosphorylation 


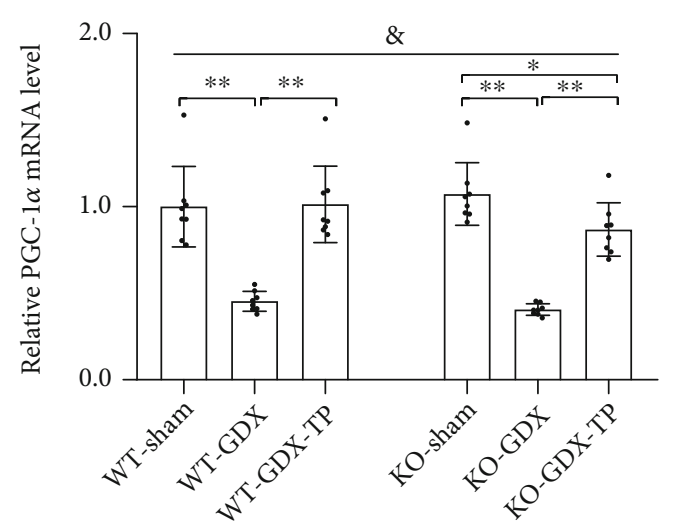

(a)

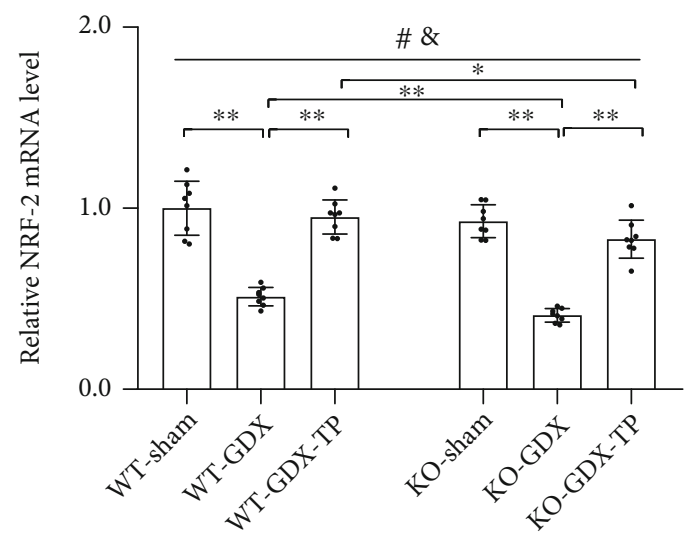

(c)

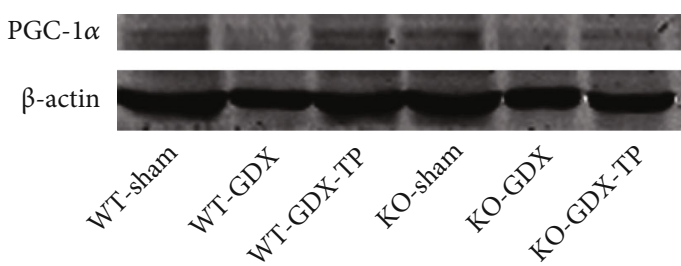

(e)

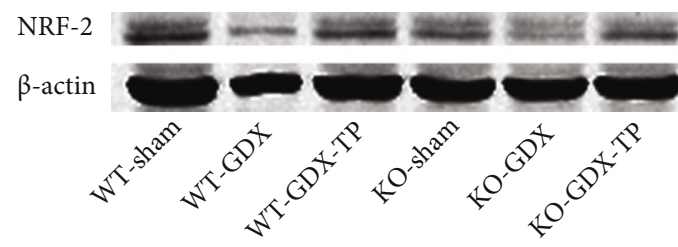

(g)

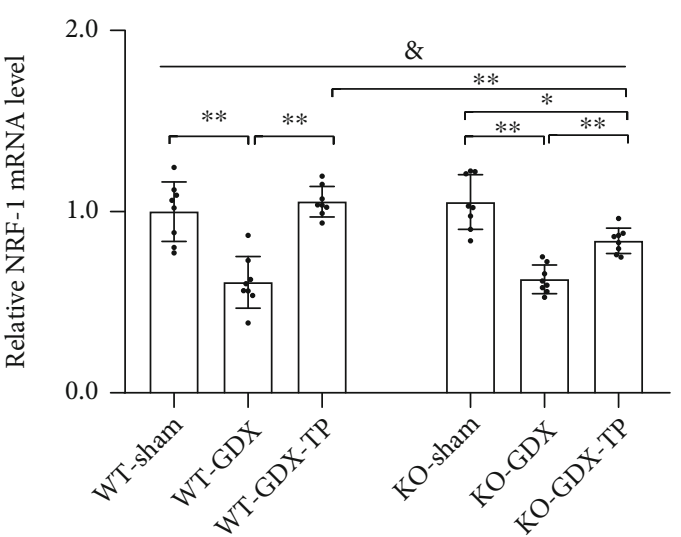

(b)

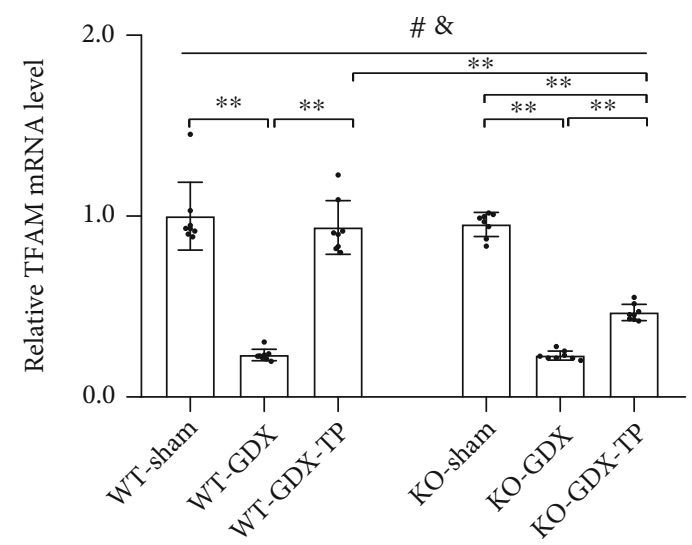

(d)

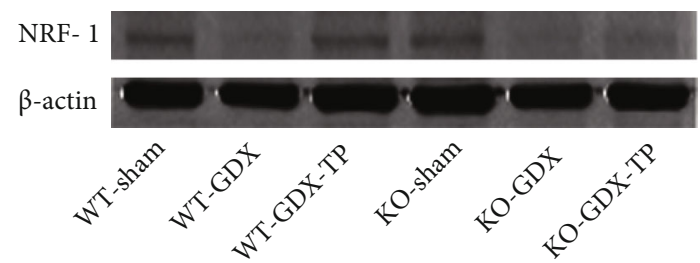

(f)

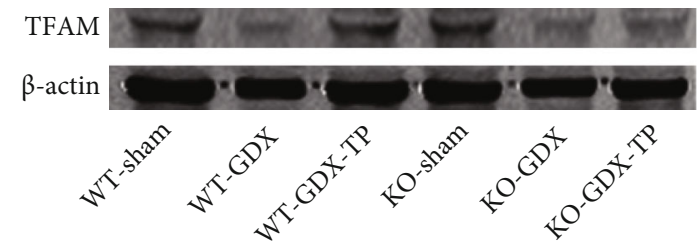

(h)

Figure 13: Continued. 


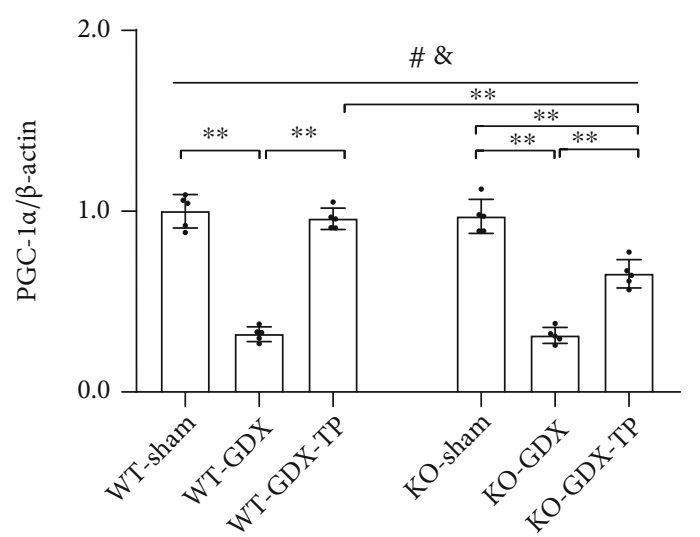

(i)

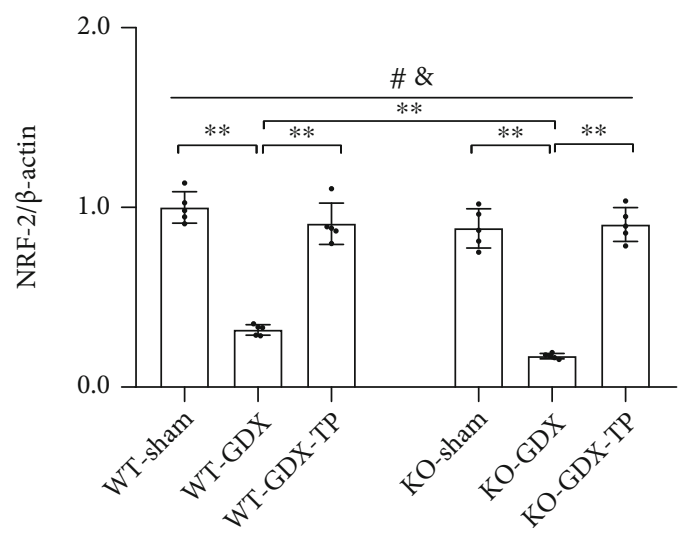

(k)

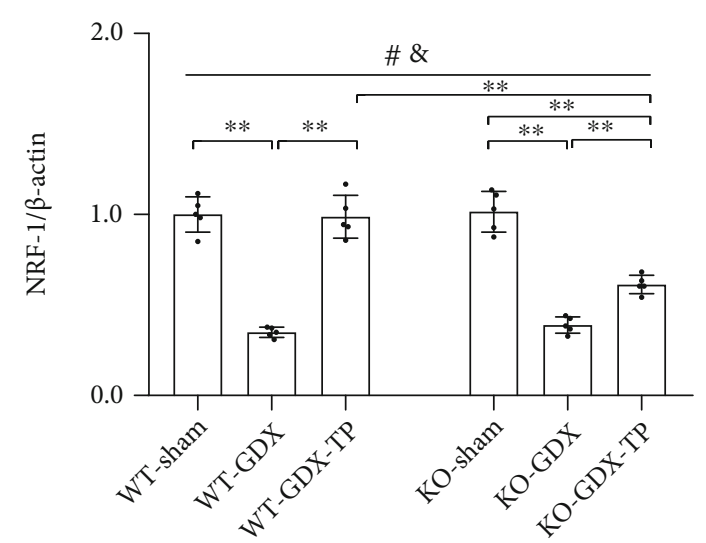

(j)

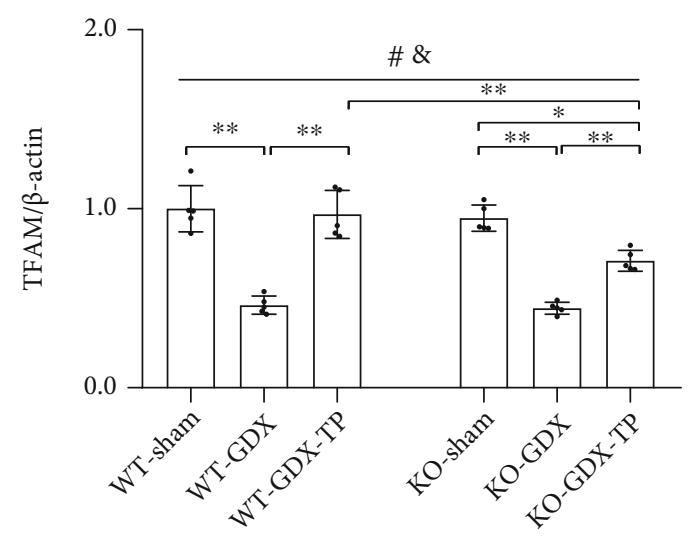

(l)

FIGURE 13: Effects of TP supplementation on mitochondrial biogenesis in the substantia nigra of Nrf2 KO GDX male mice. (a) $P G C$ - $1 \alpha$, (b) NRF-1, (c) NRF-2, and (d) TFAM mRNA were revealed by qPCR. (e, i) PGC-1 $\alpha$, (f, j) NRF-1, (g, k) NRF-2, and (h, l) TFAM proteins were detected by immunoblotting. Data were presented as mean \pm SD; $n=8$ for qPCR; $n=5$ for western blot. ${ }^{\#} P<0.05$ main effect of genotype by two-way ANOVA; ${ }^{8} P<0.05$ main effect of treatment by two-way ANOVA. ${ }^{*} P<0.05$ and ${ }^{* *} P<0.01$.

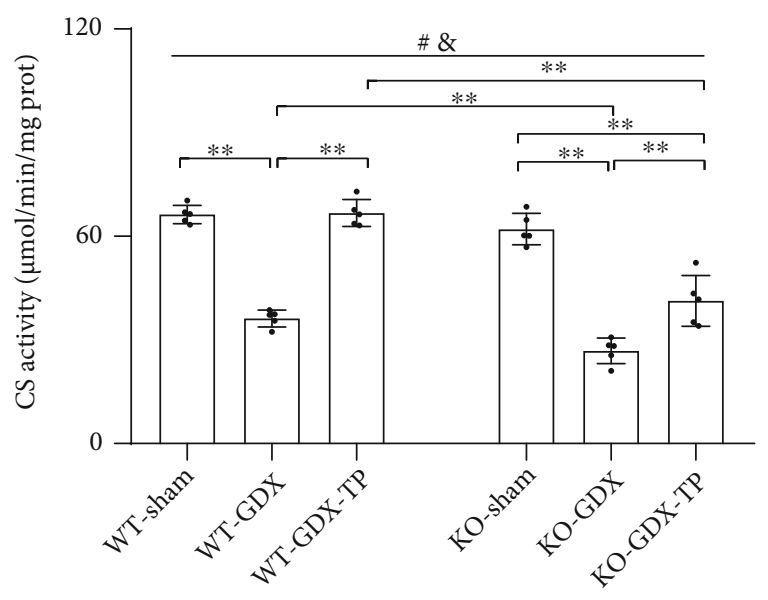

(a)

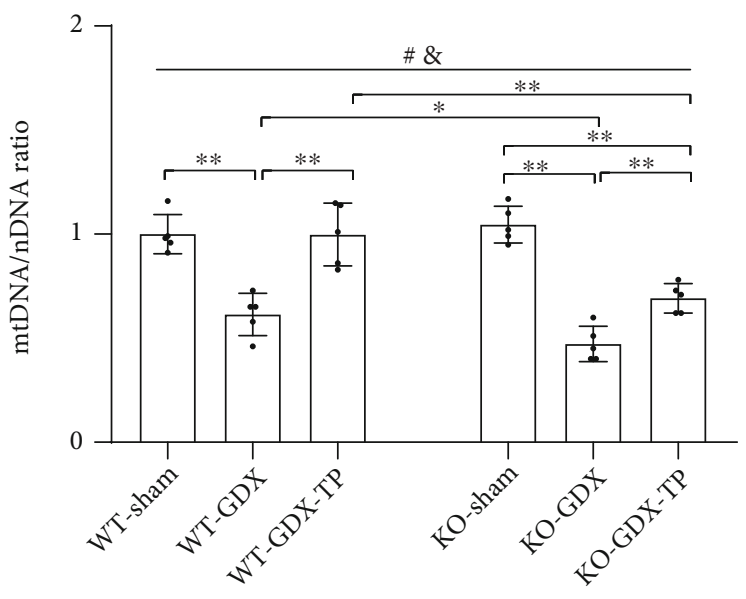

(b)

FIGURE 14: Effects of TP supplementation on mitochondrial content in the substantia nigra of Nrf2 KO GDX male mice. (a) CS activity was assessed by spectrophotometry. (b) mtDNA/nDNA was detected by qPCR. Data were presented as mean \pm SD; $n=5$. ${ }^{\#} P<0.05$ main effect of genotype by two-way ANOVA; ${ }^{\&} P<0.05$ main effect of treatment by two-way ANOVA. ${ }^{*} P<0.05$ and ${ }^{* *} P<0.01$. 


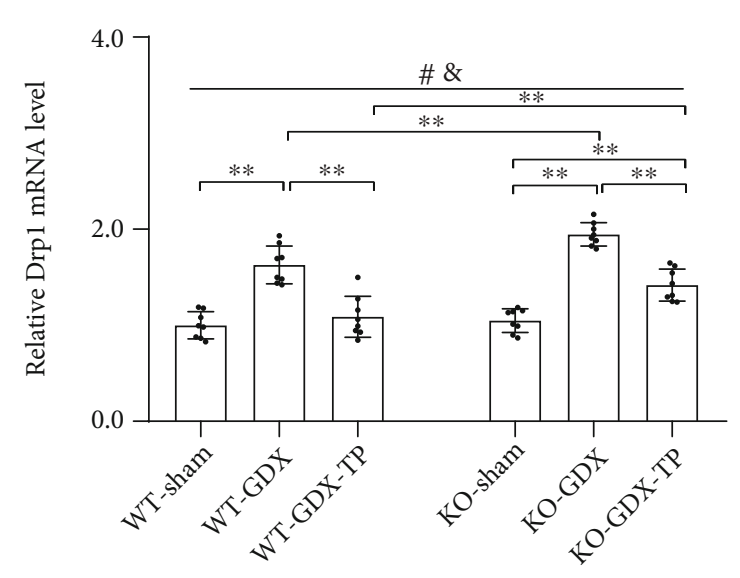

(a)

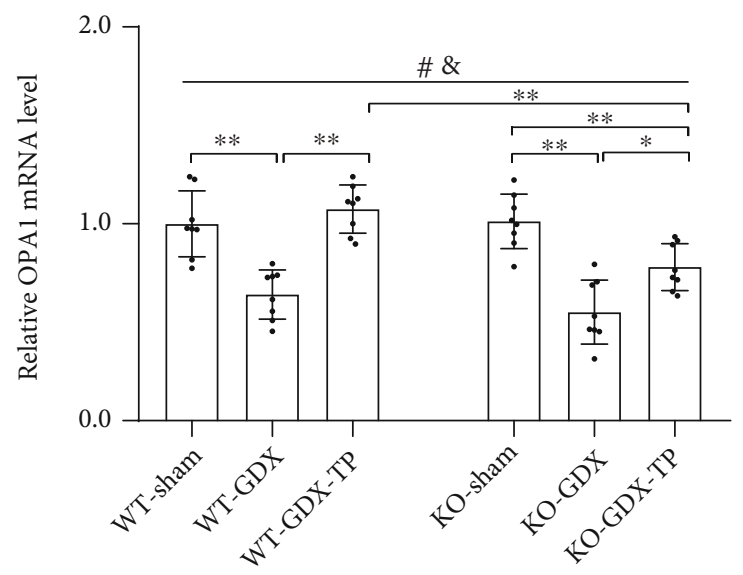

(c)

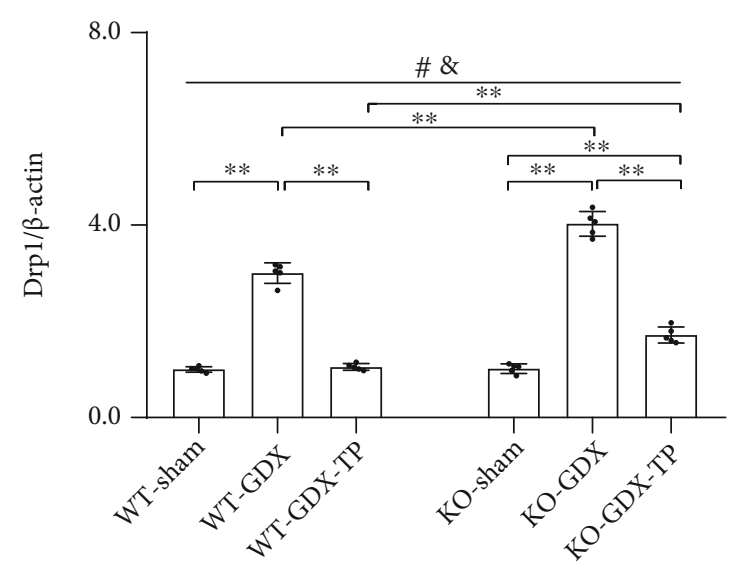

(e)

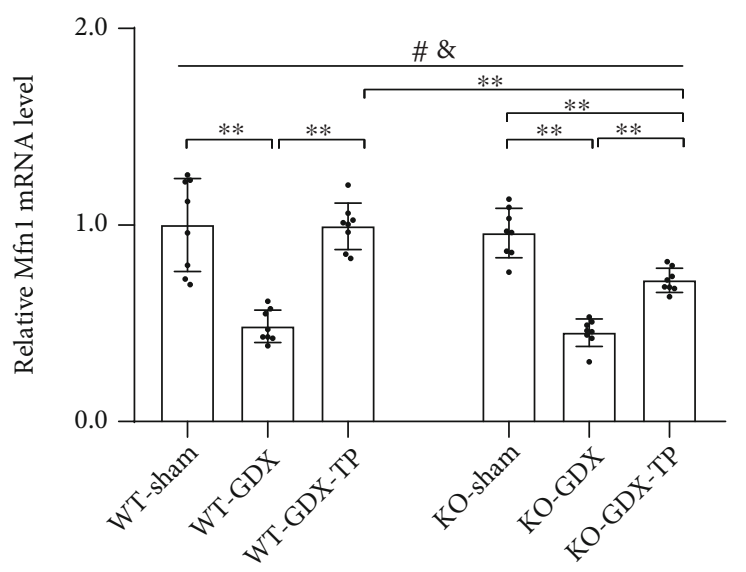

(b)

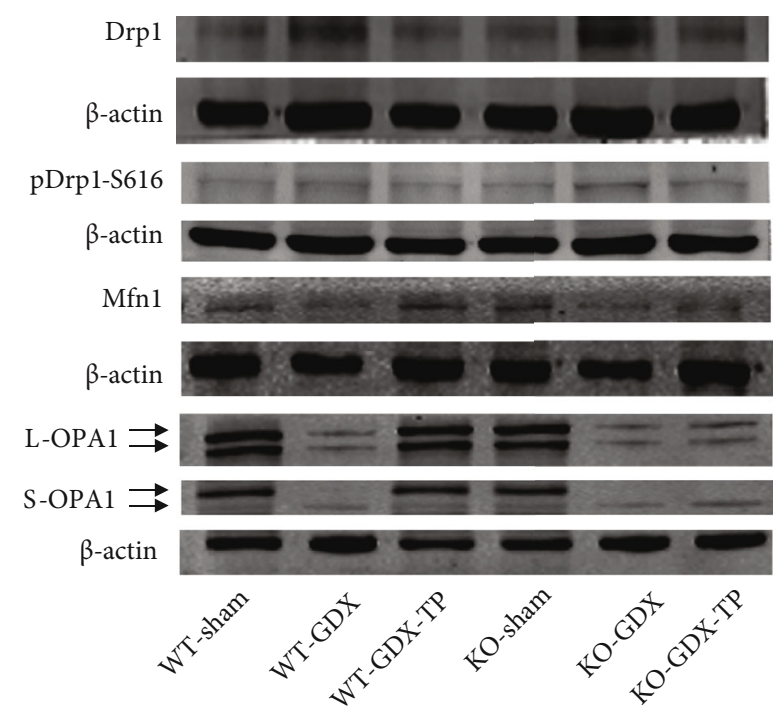

(d)

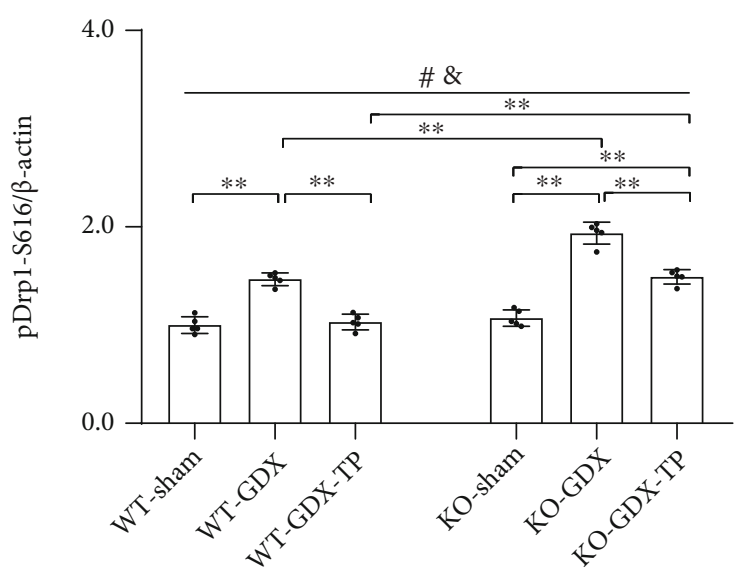

(f)

Figure 15: Continued. 


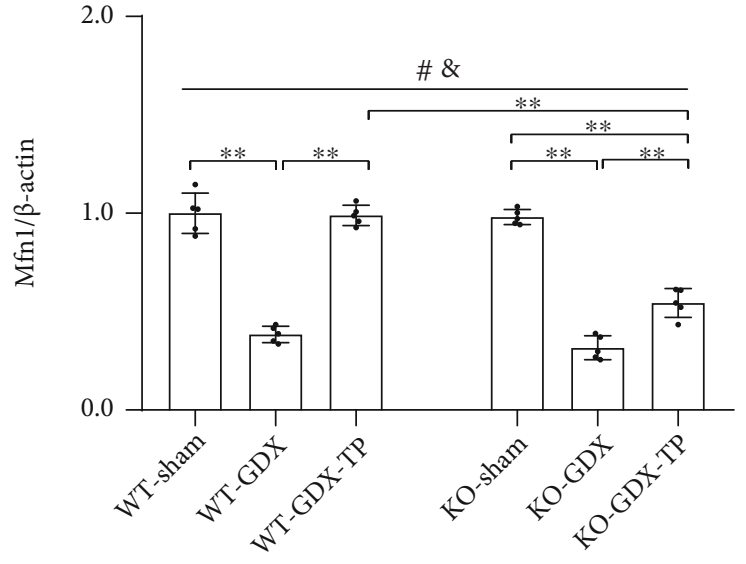

(g)

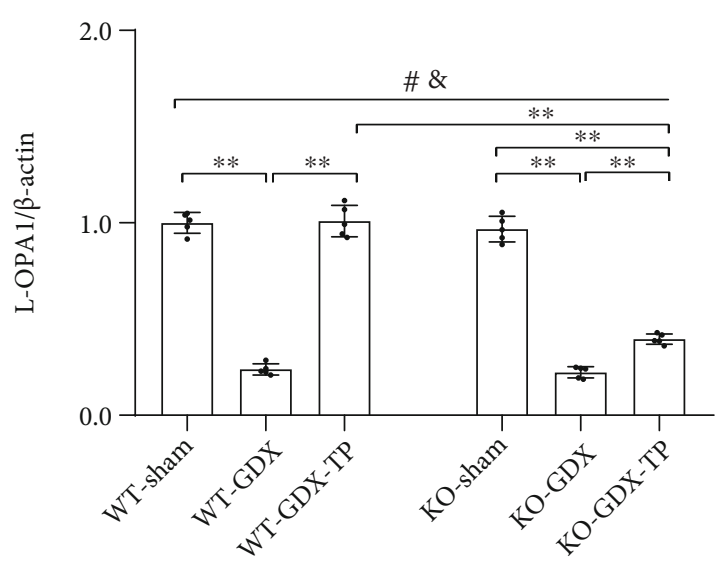

(h)

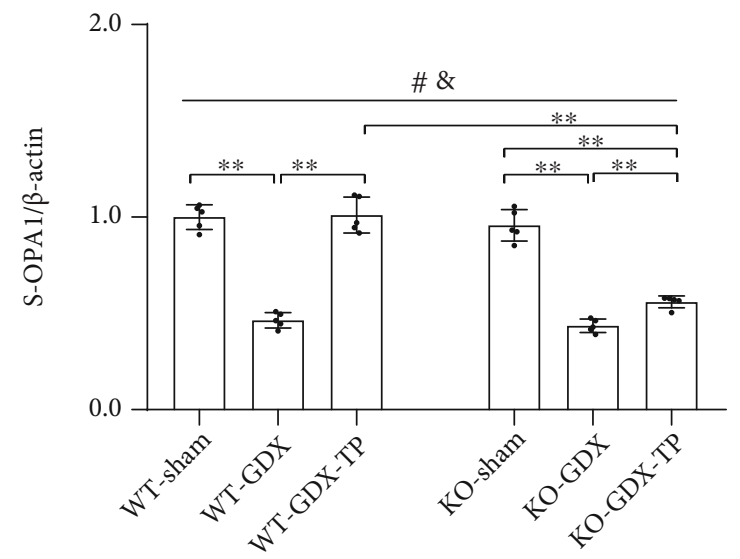

(i)

FIGURE 15: Effects of TP supplementation on mitochondrial dynamics in the substantia nigra of Nrf2 KO GDX male mice. (a) Drp1, (b) Mfn1, and (c) OPA1 mRNA were revealed by qPCR. (d, e) Drp1, (d, f) pDrp1-S616, (d, g) Mfn1, (d, h) L-OPA1, and (d, i) S-OPA1 proteins were detected by immunoblotting. Data were presented as mean $\pm \mathrm{SD} ; n=8$ for $\mathrm{qPCR} ; n=5$ for immunoblotting. ${ }^{\#} P<0.05$ main effect of genotype by two-way ANOVA; ${ }^{\circledR} P<0.05$ main effect of treatment by two-way ANOVA. ${ }^{*} P<0.05$ and ${ }^{* *} P<0.01$.

at Ser616 enhances Drp1-mediated fission [56]. Mfn1/Mfn2 and OPA1 are involved in the mitochondrial fusion [54, 55]. OPA1 activity is regulated by L-OPA1 and S-OPA1 through OPA1 cleavage. Both L-OPA1 and S-OPA1 are necessary for mitochondrial fusion; however, L-OPA1 or S-OPA1 alone is not sufficient for fusion under normal conditions [57]. The expression of Drp1 and OPA1 seemed to be affected by androgen levels. In an androgenized rat model for polycystic ovary syndrome, androgen induces upregulation of Drp1 [58]. Treatment of androgen-sensitive prostate cancer cells with an androgen receptor agonist or antagonist revealed that Drp1 is transcriptionally regulated by them [59]. In addition, it was found that testosterone increases fusion protein OPAl expression in $\mathrm{C}_{2} \mathrm{C}_{12}$ cells [43]. In the present study, we found that testosterone deficiency induced by orchiectomy disrupted balanced mitochondrial dynamics in the SN of WT young male mice. Orchiectomy increased Drp1 and pDrp1-S616 levels and decreased Mfn1, LOPA1, and S-OPA1 levels in the SN of WT young mice. It was indicated that there might be more fragmented mitochondria in the SN of GDX young male mice, which affects mitochondrial function and metabolism [51]. Unlike GDX young male mice, aged male mice showed the decreased levels of Drp1, pDrp1-S616, Mfn1, L-OPA1, and S-OPA1 in the SN. The difference related to mitochondrial dynamics between aged male mice and GDX young male mice showed that the ability of both mitochondrial fusion and mitochondrial fission was downregulated in aged male mice. Previous studies revealed the dysregulated mitochondrial dynamics in aging-related neurodegenerative diseases $[60,61]$. Specifically, increased mitochondrial fission and reduced mitochondrial fusion are major features in agingrelated neurodegenerative diseases $[60,62]$. However, in contrast with the different expression state between fission proteins and fusion proteins in pathological conditions, natural aging shows the declined expression of both fission proteins and fusion proteins $[63,64]$. Mitochondrial fission-fusion mRNA and protein expression levels are differentially altered in the aging process, but they reach significantly lower levels of mitochondrial dynamics proteins in older animals [64]. Decreased Drp1 was found in several aged mouse tissues including neurons, in cultured aged human endothelial cells, or in old human skeletal muscle [63-65], and reduced Mfn1 and OPA1 were also detected in the skeletal muscle of aged 
mice $[64,65]$. The balance between mitochondrial fusion and fission is clearly crucial for neuronal function [51]. Therefore, aged organisms might try to maintain a balanced mitochondrial dynamics through downregulating both fission and fusion protein levels. However, altered mitochondrial dynamics by downregulating mitochondrial dynamics proteins in the aging process might not be efficient enough to maintain normal mitochondrial structures and functions in aged animals $[65,66]$ as young animals do. Supplementation with TP upregulated Drp1 and pDrp1-S616 involved in fission, as well as Mfn1, L-OPA1, and S-OPA1 related to fusion in the SN of WT-aged male mice, thus ameliorating their mitochondrial dynamics in the SN. However, this effect of TP supplementation in WT-aged mice was not detected in Nrf2 KO-aged male mice. Nrf2 deficiency blocked efficiency of TP supplementation in aged male mice. The present results indicated that the regulation of mitochondrial dynamics in aged male mice was affected by testosterone levels and Nrf2 was a key factor for TP upregulation of mitochondrial dynamics in the $\mathrm{SN}$ of aged male mice. Mitochondrial fusion contributes to increasing mitochondrial ATP production [67]; therefore, the increased ATP levels, as well as improved status of nigrostriatal dopaminergic neurons in the SN of TP-treated WT-aged male mice, suggested that the final net effects of altered mitochondrial dynamics by TP supplementation might promote mitochondrial fusion, which should be testified in the future studies.

Both testosterone level declines and age-related mitochondrial dysfunction are present in aging men and rodents $[18,46,68]$. Coincidentally, Nrf2 also decreases during aging process [69]. It seemed that there was close association among them. An age-related decline in Nrf2 function might play an important role between loss of testosterone and declined mitochondrial function during aging process. It was found that Nrf2 is involved in the maintenance of mitochondrial function through modulating cellular redox balance and MMP [70]. Activation of Nrf2 would contribute to the improved mitochondrial function during aging process. In the present study, we found that supplementation with TP increased Nrf2, as well as its downstream target HO-1 expression, and promoted Nrf2 nuclear translocation in the aged male mice. Thus, loss of testosterone during aging process might reduce activation of Nrf2, affecting mitochondrial function. Ameliorated mitochondrial function in the SN of WT-aged-TP mice was related to Nrf2 activation by TP supplementation to large extent. Nrf2 deficiency exacerbated mitochondrial dysfunction in the SN of aging male mice, and TP supplementation did not ameliorate the exacerbated mitochondrial dysfunction in $\mathrm{KO}$-aged male mice. The following might explain the difference of mitochondrial function in the SN of TP-supplemented aged male mice of both genotypes. One was direct involvement of Nrf2 in the regulation of mitochondrial function in this process. The study by Piantadosi et al. checked for Nrf2-binding sites in the NRF-1 promoter and found four AREs in the NRF-1 gene promoter [71]. Nrf2 binds to AREs of NRF-1 promoter, activates NRF-1, and induces mitochondrial biogenesis via TFAM [71]. TP supplementation to aged male mice increased nuclear Nrf2 levels in the SN, thus improving mito- chondrial function of aged male mice through promoting mitochondrial biogenesis. This effect of TP supplementation was significantly blocked due to Nrf2 knockout. Another was the oxidative stress milieus of cells, whether it was ameliorated by the regulation of antioxidants and antioxidant gene expression. The oxidative stress status among experimental groups was different in the present study. Significantly increased oxidative stress was present in $\mathrm{KO}$-aged mice, as well as KO-aged-TP mice, compared with WT-aged mice. Testosterone has protective effects in a low oxidative stress environment [22]. And these protective effects will be weakened or even harmful in the state of high oxidative stress [22, 72]. In addition, by comparing the studies on castration and androgen replacement in young male mice, we also found that the effects of TP supplementation were significantly weaker in KO-GDX mice than WT-GDX mice, and the oxidative stress state of the SN was significantly lower in WTGDX mice than KO-GDX mice. Thus, the present results that Nrf2 deficiency attenuated the efficiency of TP supplementation was to a certain extent due to high oxidative stress state. Activation of Nrf2 to reduce oxidative stress was beneficial for testosterone to play its ameliorative effects on mitochondrial dysfunction in aging and aged-related neurodegenerative disorders in males. Considering the involvement of $\mathrm{Nrf2}$ in regulating mitochondrial functions, it was presumed that Nrf2 might also affect mitochondrial dynamics through direct or indirect way in TP supplementation to aged male mice. However, direct experimental evidence for Nrf2 roles in mitochondrial dynamics is limited [11]. So, it was unclear to what extent changes in mitochondrial dynamics in the present studies were involved in the improvement of mitochondrial function by TP. The changed mitochondrial dynamics in TP-treated aged male mice was causal in improved mitochondrial function, or merely a downstream consequence should be further clarified in the future study.

\section{Conclusion}

In summary, Nrf2 knockout further reduced the nigrostriatal dopaminergic neurochemical content and aggravated downregulation of both mitochondrial biogenesis and mitochondrial dynamics in the substantia nigra upon aging. Supplementation with testosterone induced the amelioration on nigrostriatal dopaminergic activity and mitochondrial function in aged male animals by modulation of mitochondrial biogenesis and mitochondrial dynamics. Nrf2 deficiency blocked testosterone-induced upregulation of mitochondrial biogenesis and mitochondrial dynamics in the aging substantia nigra. Thus, as a target, activation of Nrf2 might be conducive to testosterone-upregulating mitochondrial biogenesis and mitochondrial dynamics in the substantia nigra upon aging to produce mitochondria that are more efficient in ATP production and have optimal oxidative capacity.

\section{Data Availability}

The data that support the findings of this study are available from the corresponding author upon request. 


\section{Conflicts of Interest}

The authors declare that they have no conflicts of interest.

\section{Authors' Contributions}

BR, TZ, QG, JC, and YK performed the experiments. BR and TZ wrote the manuscript draft. YW, RC, XJ, and GZ analyzed the data. GS designed the experiments and revised the manuscript. All authors approved the final version of the manuscript. Baoliang Ren and Tianyun Zhang contributed equally to this work.

\section{Acknowledgments}

This project was financially supported by the National Natural Science Foundation of China (No. 81871119), Natural Science Foundation of Hebei Province of China (No. C2017206072), and Postgraduate Student Innovation Ability Training Foundation of Hebei Education Department (No. CXZZBS2021071).

\section{Supplementary Materials}

Table S1: accession numbers of the genes for primers. (Supplementary Materials)

\section{References}

[1] K. F. Winklhofer and C. Haass, "Mitochondrial dysfunction in Parkinson's disease," Biochimica et Biophysica Acta, vol. 1802, pp. 29-44, 2010.

[2] T. R. Figueira, M. H. Barros, A. A. Camargo et al., "Mitochondria as a source of reactive oxygen and nitrogen species: from molecular mechanisms to human health," Antioxidants \& Redox Signaling, vol. 18, no. 16, pp. 2029-2074, 2013.

[3] V. Sorrentino, K. J. Menzies, and J. Auwerx, "Repairing mitochondrial dysfunction in disease," Annual Review of Pharmacology and Toxicology, vol. 58, pp. 353-389, 2018.

[4] P. A. Li, X. Hou, and S. Hao, "Mitochondrial biogenesis in neurodegeneration," Journal of Neuroscience Research, vol. 95, pp. 2025-2029, 2017.

[5] X. Zhou, H. Chen, L. Wang et al., "Mitochondrial dynamics: a potential therapeutic target for ischemic stroke," Frontiers in Aging Neuroscience, vol. 13, article 721428, 2021.

[6] M. Uittenbogaard and A. Chiaramello, "Mitochondrial biogenesis: a therapeutic target for neurodevelopmental disorders and neurodegenerative diseases," Current Pharmaceutical Design, vol. 20, pp. 5574-5593, 2014.

[7] M. Khacho and R. S. Slack, "Mitochondrial dynamics in the regulation of neurogenesis: from development to the adult brain," Developmental Dynamics, vol. 247, pp. 47-53, 2018.

[8] J. St-Pierre, S. Drori, M. Uldry et al., "Suppression of reactive oxygen species and neurodegeneration by the PGC-1 transcriptional coactivators," Cell, vol. 127, pp. 397-408, 2006.

[9] P. C. Chen, M. R. Vargas, A. K. Pani et al., "Nrf2-mediated neuroprotection in the MPTP mouse model of Parkinson's disease: critical role for the astrocyte," Proceedings of the National Academy of Sciences of the United States of America, vol. 106, pp. 2933-2938, 2009.
[10] A. P. Gureev, E. A. Shaforostova, and V. N. Popov, "Regulation of mitochondrial biogenesis as a way for active longevity: interaction between the Nrf2 and PGC- $1 \alpha$ signaling pathways," Frontiers in Genetics, vol. 10, p. 435, 2019.

[11] T. C. Kang, "Nuclear factor-erythroid 2-related factor 2 (Nrf2) and mitochondrial dynamics/mitophagy in neurological diseases," Antioxidants (Basel), vol. 9, p. 617, 2020.

[12] J. X. Liu, C. Yang, Z. J. Liu et al., "Protection of procyanidin B2 on mitochondrial dynamics in sepsis associated acute kidney injury via promoting Nrf2 nuclear translocation," Aging (Albany NY), vol. 12, pp. 15638-15655, 2020.

[13] L. Yang, X. Li, A. Jiang et al., "Metformin alleviates lead-induced mitochondrial fragmentation via AMPK/Nrf2 activation in SHSY5Y cells," Redox Biology, vol. 36, article 101626, 2020.

[14] R. Cui, G. Zhang, Y. Kang et al., “Amelioratory effects of testosterone propionate supplement on behavioral, biochemical and morphological parameters in aged rats," Experimental Gerontology, vol. 47, pp. 67-76, 2012.

[15] G. Zhang, S. Li, Y. Kang et al., "Enhancement of dopaminergic activity and region-specific activation of Nrf2-ARE pathway by intranasal supplements of testosterone propionate in aged male rats," Hormones and Behavior, vol. 80, pp. 103-116, 2016.

[16] M. S. Okun, W. M. McDonald, and M. R. DeLong, "Refractory nonmotor symptoms in male patients with Parkinson disease due to testosterone deficiency: a common unrecognized comorbidity," Archives of Neurology, vol. 59, no. 5, pp. 807-811, 2002.

[17] F. Wang, J. Yang, J. Sun et al., "Testosterone replacement attenuates mitochondrial damage in a rat model of myocardial infarction," The Journal of Endocrinology, vol. 225, no. 2, pp. 101-111, 2015.

[18] W. Yan, T. Zhang, Y. Kang et al., "Testosterone ameliorates age-related brain mitochondrial dysfunction," Aging (Albany NY), vol. 13, pp. 16229-16247, 2021.

[19] T. Hioki, S. Suzuki, M. Morimoto et al., "Brain testosterone deficiency leads to down-regulation of mitochondrial gene expression in rat hippocampus accompanied by a decline in peroxisome proliferator-activated receptor- $\gamma$ coactivator $1 \alpha$ expression," Journal of Molecular Neuroscience, vol. 52, pp. 531-537, 2014.

[20] W. Yan, Y. Kang, X. Ji et al., "Testosterone upregulates the expression of mitochondrial ND1 and ND4 and alleviates the oxidative damage to the nigrostriatal dopaminergic system in orchiectomized rats," Oxidative Medicine and Cellular Longevity, vol. 2017, Article ID 1202459, 13 pages, 2017.

[21] M. T. Lin and M. F. Beal, "Mitochondrial dysfunction and oxidative stress in neurodegenerative diseases," Nature, vol. 443, pp. 787-795, 2006.

[22] S. Holmes, B. Abbassi, C. Su, M. Singh, and R. L. Cunningham, "Oxidative stress defines the neuroprotective or neurotoxic properties of androgens in immortalized female rat dopaminergic neuronal cells," Endocrinology, vol. 154, pp. 42814292, 2013.

[23] G. Zhang, G. Shi, H. Tan, Y. Kang, and H. Cui, "Intranasal administration of testosterone increased immobile-sniffing, exploratory behavior, motor behavior and grooming behavior in rats," Hormones and Behavior, vol. 59, pp. 477-483, 2011.

[24] Y. Kang, W. Yan, H. Fang et al., "Alleviation of oxidative damage and involvement of Nrf2-ARE pathway in mesodopaminergic system and hippocampus of status epilepticus rats pretreated by intranasal pentoxifylline," Oxidative Medicine and Cellular Longevity, vol. 2017, Article ID 7908072, 18 pages, 2017. 
[25] Y. Wang, Y. Kang, C. Qi et al., "Pentoxifylline enhances antioxidative capability and promotes mitochondrial biogenesis for improving age-related behavioral deficits," Aging (Albany NY), vol. 12, pp. 25487-25504, 2020.

[26] L. Liu, L. F. Zuo, J. Zuo, and J. Wang, "Artesunate induces apoptosis and inhibits growth of Eca109 and Ec9706 human esophageal cancer cell lines in vitro and in vivo," Molecular Medicine Reports, vol. 12, pp. 1465-1472, 2015.

[27] A. Björklund and S. B. Dunnett, "Dopamine neuron systems in the brain: an update," Trends in Neurosciences, vol. 30, pp. 194-202, 2007.

[28] N. A. Harrison, M. Cercignani, V. Voon, and H. D. Critchley, "Effects of inflammation on hippocampus and substantia nigra responses to novelty in healthy human participants," Neuropsychopharmacology, vol. 40, pp. 831-838, 2015.

[29] H. L. Sanchez, L. B. Silva, E. L. Portiansky, C. B. Herenu, R. G. Goya, and G. O. Zuccolilli, "Dopaminergic mesencephalic systems and behavioral performance in very old rats," Neuroscience, vol. 154, pp. 1598-1606, 2008.

[30] B. Sheng, X. Wang, B. Su et al., "Impaired mitochondrial biogenesis contributes to mitochondrial dysfunction in Alzheimer's disease," Journal of Neurochemistry, vol. 120, pp. 419429, 2012.

[31] M. E. Emborg, S. Y. Ma, E. J. Mufson et al., "Age-related declines in nigral neuronal function correlate with motor impairments in rhesus monkeys," The Journal of Comparative Neurology, vol. 401, no. 2, pp. 253-265, 1998.

[32] M. J. Kurz, K. Pothakos, S. Jamaluddin, M. Scott-Pandorf, C. Arellano, and Y. S. Lau, "A chronic mouse model of Parkinson's disease has a reduced gait pattern certainty," Neuroscience Letters, vol. 429, no. 1, pp. 39-42, 2007.

[33] C. A. Frye, K. Edinger, and K. Sumida, "Androgen administration to aged male mice increases anti-anxiety behavior and enhances cognitive performance," Neuropsychopharmacology, vol. 33, pp. 1049-1061, 2008.

[34] E. Mitchell, D. Thomas, and R. Burnet, "Testosterone improves motor function in Parkinson's disease," Journal of Clinical Neuroscience, vol. 13, no. 1, pp. 133-136, 2006.

[35] A. Giudice and M. Montella, "Activation of the Nrf2-ARE signaling pathway: a promising strategy in cancer prevention," BioEssays, vol. 28, pp. 169-181, 2006.

[36] C. P. Ramsey, C. A. Glass, M. B. Montgomery et al., "Expression of Nrf2 in neurodegenerative diseases," Journal of Neuropathology and Experimental Neurology, vol. 66, pp. 75-85, 2007.

[37] J. T. Coyle and P. Puttfarcken, "Oxidative stress, glutamate, and neurodegenerative disorders," Science, vol. 262, pp. 689695, 1993.

[38] H. F. Poon, V. Calabrese, G. Scapagnini, and D. A. Butterfield, "Free radicals and brain aging," Clinics in Geriatric Medicine, vol. 20, pp. 329-359, 2004.

[39] M. Bandookwala and P. Sengupta, “3-Nitrotyrosine: a versatile oxidative stress biomarker for major neurodegenerative diseases," The International Journal of Neuroscience, vol. 130, pp. 1047-1062, 2020.

[40] D. Teixeira, R. Fernandes, C. Prudêncio, and M. Vieira, "3Nitrotyrosine quantification methods: current concepts and future challenges," Biochimie, vol. 125, pp. 1-11, 2016.

[41] P. Puigserver and B. M. Spiegelman, "Peroxisome proliferatoractivated Receptor- $\gamma$ coactivator $1 \alpha$ (PGC- $1 \alpha)$ : transcriptional coactivator and metabolic regulator," Endocrine Reviews, vol. 24, no. 1, pp. 78-90, 2003.
[42] R. C. Scarpulla, "Nuclear respiratory factors and the pathways of nuclear-mitochondrial interaction," Trends in Cardiovascular Medicine, vol. 6, pp. 39-45, 1996.

[43] L. Pronsato, L. Milanesi, and A. Vasconsuelo, "Testosterone induces up-regulation of mitochondrial gene expression in murine $\mathrm{C} 2 \mathrm{C} 12$ skeletal muscle cells accompanied by an increase of nuclear respiratory factor-1 and its downstream effectors," Molecular and Cellular Endocrinology, vol. 500, article 110631, 2020.

[44] T. Usui, K. Kajita, T. Kajita et al., "Elevated mitochondrial biogenesis in skeletal muscle is associated with testosteroneinduced body weight loss in male mice," FEBS Letters, vol. 588, pp. 1935-1941, 2014.

[45] Y. Kim, M. Triolo, and D. A. Hood, "Impact of aging and exercise on mitochondrial quality control in skeletal muscle," Oxidative Medicine and Cellular Longevity, vol. 2017, Article ID 3165396, 16 pages, 2017.

[46] C. Kang, E. Chung, G. Diffee, and L. L. Ji, "Exercise training attenuates aging-associated mitochondrial dysfunction in rat skeletal muscle: role of PGC-1 $\alpha$," Experimental Gerontology, vol. 48, pp. 1343-1350, 2013.

[47] M. Narasimhan, J. Hong, N. Atieno et al., "Nrf2 deficiency promotes apoptosis and impairs PAX7/MyoD expression in aging skeletal muscle cells," Free Radical Biology \& Medicine, vol. 71, pp. 402-414, 2014.

[48] T. Cayci, Y. G. Kurt, E. O. Akgul, and B. Kurt, "Does mtDNA copy number mean mitochondrial abundance?," Journal of Assisted Reproduction and Genetics, vol. 29, no. 8, p. 855, 2012.

[49] Q. Ye, C. Chen, E. Si et al., "Mitochondrial effects of PGC1alpha silencing in MPP+ treated human SH-SY5Y neuroblastoma cells," Frontiers in Molecular Neuroscience, vol. 10, p. 164, 2017.

[50] M. Liesa, M. Palacín, and A. Zorzano, "Mitochondrial dynamics in mammalian health and disease," Physiological Reviews, vol. 89, pp. 799-845, 2009.

[51] D. Sebastián, M. Palacín, and A. Zorzano, "Mitochondrial dynamics: coupling mitochondrial fitness with healthy aging," Trends in Molecular Medicine, vol. 23, pp. 201-215, 2017.

[52] R. J. Youle and A. M. van der Bliek, "Mitochondrial fission, fusion, and stress," Science, vol. 337, pp. 1062-1065, 2012.

[53] N. Ishihara, M. Nomura, A. Jofuku et al., "Mitochondrial fission factor Drp1 is essential for embryonic development and synapse formation in mice," Nature Cell Biology, vol. 11, pp. 958-966, 2009.

[54] H. Chen, S. A. Detmer, A. J. Ewald, E. E. Griffin, S. E. Fraser, and D. C. Chan, "Mitofusins Mfn1 and Mfn2 coordinately regulate mitochondrial fusion and are essential for embryonic development," The Journal of Cell Biology, vol. 160, pp. 189200, 2003.

[55] Z. Song, M. Ghochani, J. M. McCaffery, T. G. Frey, and D. C. Chan, "Mitofusins and OPA1 mediate sequential steps in mitochondrial membrane fusion," Molecular Biology of the Cell, vol. 20, pp. 3525-3532, 2009.

[56] T. Bo, T. Yamamori, M. Suzuki, Y. Sakai, K. Yamamoto, and O. Inanami, "Calmodulin-dependent protein kinase II (CaMKII) mediates radiation-induced mitochondrial fission by regulating the phosphorylation of dynamin-related protein 1 (Drp1) at serine 616," Biochemical and Biophysical Research Communications, vol. 495, no. 2, pp. 1601-1607, 2018. 
[57] Z. Song, H. Chen, M. Fiket, C. Alexander, and D. C. Chan, "OPA1 processing controls mitochondrial fusion and is regulated by mRNA splicing, membrane potential, and Yme1L," The Journal of Cell Biology, vol. 178, no. 5, pp. 749-755, 2007.

[58] R. Salehi, H. L. Mazier, A. L. Nivet et al., "Ovarian mitochondrial dynamics and cell fate regulation in an androgeninduced rat model of polycystic ovarian syndrome," Scientific Reports, vol. 10, p. 1021, 2020.

[59] V. Choudhary, I. Kaddour-Djebbar, V. Lakshmikanthan et al., "Novel role of androgens in mitochondrial fission and apoptosis," Molecular Cancer Research, vol. 9, pp. 1067-1077, 2011.

[60] X. Wang, B. Su, S. L. Siedlak et al., “Amyloid-beta overproduction causes abnormal mitochondrial dynamics via differential modulation of mitochondrial fission/fusion proteins," Proceedings of the National Academy of Sciences of the United States of America, vol. 105, pp. 19318-19323, 2008.

[61] X. Wang, B. Su, H. G. Lee et al., "Impaired balance of mitochondrial fission and fusion in Alzheimer's disease," The Journal of Neuroscience, vol. 29, pp. 9090-9103, 2009.

[62] P. H. Reddy, T. P. Reddy, M. Manczak, M. J. Calkins, U. Shirendeb, and P. Mao, "Dynamin-related protein 1 and mitochondrial fragmentation in neurodegenerative diseases," Brain Research Reviews, vol. 67, pp. 103-118, 2011.

[63] S. Mai, M. Klinkenberg, G. Auburger, J. Bereiter-Hahn, and M. Jendrach, "Decreased expression of Drp1 and Fis1 mediates mitochondrial elongation in senescent cells and enhances resistance to oxidative stress through PINK1," Journal of Cell Science, vol. 123, pp. 917-926, 2010.

[64] A. Del Campo, I. Contreras-Hernández, M. Castro-Sepúlveda et al., "Muscle function decline and mitochondria changes in middle age precede sarcopenia in mice," Aging (Albany NY), vol. 10, pp. 34-55, 2018.

[65] J. D. Crane, M. C. Devries, A. Safdar, M. J. Hamadeh, and M. A. Tarnopolsky, "The effect of aging on human skeletal muscle mitochondrial and intramyocellular lipid ultrastructure," The Journals of Gerontology. Series A, Biological Sciences and Medical Sciences, vol. 65, no. 2, pp. 119-128, 2010.

[66] K. L. Stauch, P. R. Purnell, and H. S. Fox, “Aging synaptic mitochondria exhibit dynamic proteomic changes while maintaining bioenergetic function," Aging (Albany NY), vol. 6, pp. 320-334, 2014.

[67] C. H. Yao, R. Wang, Y. Wang, C. P. Kung, J. D. Weber, and G. J. Patti, "Mitochondrial fusion supports increased oxidative phosphorylation during cell proliferation," eLife, vol. 8, article e41351, 2019.

[68] E. Fabbri, Y. An, M. Gonzalez-Freire et al., "Bioavailable testosterone linearly declines over a wide age spectrum in men and women from the Baltimore longitudinal study of aging," The Journals of Gerontology. Series A, Biological Sciences and Medical Sciences, vol. 71, no. 9, pp. 1202-1209, 2016.

[69] H. Zhang, K. J. A. Davies, and H. J. Forman, “Oxidative stress response and Nrf2 signaling in aging," Free Radical Biology \& Medicine, vol. 88, no. Part B, pp. 314-336, 2015.
[70] A. T. Dinkova-Kostova and A. Y. Abramov, "The emerging role of Nrf2 in mitochondrial function," Free Radical Biology \& Medicine, vol. 88, no. Part B, pp. 179-188, 2015.

[71] C. A. Piantadosi, M. S. Carraway, A. Babiker, and H. B. Suliman, "Heme oxygenase-1 regulates cardiac mitochondrial biogenesis via Nrf2-mediated transcriptional control of nuclear respiratory factor-1," Circulation Research, vol. 103, no. 11, pp. 1232-1240, 2008.

[72] R. Cui, Y. Kang, L. Wang et al., "Testosterone propionate exacerbates the deficits of nigrostriatal dopaminergic system and downregulates Nrf2 expression in reserpine-treated aged male rats," Frontiers in Aging Neuroscience, vol. 9, p. 172, 2017. 